\title{
Aromaticity-dependent Regioselectivity in Pd(II)-catalyzed C-H Direct Arylation of Aryl Ureas
}

Pingping Jiang, ${ }^{\dagger}$ Feng Li, ${ }^{\dagger}$ Yongbao Xu, Qingwen Liu, Jing Wang, Hong Ding, Renfu Yu and Qifeng Wang*

\section{Table of cotents}
(A) General information.
(B) Optimization of the reaction conditions for $\mathrm{C}-\mathrm{H}$ bond direct arylation. $\mathrm{S} 2$
(C) General procedure for direct arylation. S3
(D) $\quad{ }^{1} \mathrm{H}$ and ${ }^{13} \mathrm{C}$ NMR data of isolated compounds. S3
(E) Crystallographic data for 3ah. $\mathrm{S} 10$
(F) Copies of ${ }^{1} \mathrm{H}$ and ${ }^{13} \mathrm{C}$ spectra S25 


\section{(A) General information}

Unless otherwise noted, all the reactions were performed under air atmosphere. All reagents were used without purification as commercially available. All reactions were monitored by thin layer chromatography. Purification of reaction products were carried out by flash chromatography on silica gel. Chemical yields refer to pure isolated substances. The melting point was recorded on a melting point apparatus (MPA100, Stanford Research Systems, Inc.). ${ }^{1} \mathrm{H}$ NMR and ${ }^{13} \mathrm{C}$ NMR spectra were recorded on Bruker $300 \mathrm{MHz}$ spectrometers (300 MHz for ${ }^{1} \mathrm{H}$ NMR and $75 \mathrm{MHz}$ for ${ }^{13} \mathrm{C}$ NMR). Chemical shifts of ${ }^{1} \mathrm{H}$ and ${ }^{13} \mathrm{C}$ signals were given in $\delta$ relative to the solvents residual ${ }^{1} \mathrm{H}$-signal $\left(\mathrm{CH}_{-} \mathrm{Cl}_{3}, \delta(\mathrm{H}) 7.26\right.$, DMSO, $\left.\delta(\mathrm{H}) 2.50\right)$ or to $\mathrm{Me}_{4} \mathrm{Si}(\delta 0.0) . \mathrm{CDCl} 3$ resonance in the ${ }^{13} \mathrm{C}$ spectrum is $77.2 \mathrm{ppm}$ and DMSO resonance in the ${ }^{13} \mathrm{C}$ spectrum is $39.5 \mathrm{ppm}$. The following abbreviations are used: s, singlet, d, doublet, t, triplet, q, quartet, quint, quintuplet, m, multiplet, br, broad. High-resolution mass spectral analysis (HRMS) data were measured on a Bruker ApexII mass spectrometer by means of the ESI technique.

\section{(B) Optimization of the reaction conditions for $\mathrm{C}-\mathrm{H}$ bond direct arylation.}

1,1-Dimethyl-3-(naphthalen-2-yl)urea (42.8 mg, $0.2 \mathrm{mmol})$ 1a, o-methyl-phenyl boronic acid (40.8 mg, 0.3 mmol or $81.6 \mathrm{mg}, 0.6 \mathrm{mmol}) \mathbf{2 a}, \mathrm{Cu}(\mathrm{OAc})_{2} \cdot \mathrm{H}_{2} \mathrm{O}(39.9 \mathrm{mg}, 0.2 \mathrm{mmol}), \mathrm{PhCO}_{3} \mathrm{Bu}-t(77.7 \mathrm{mg}, 0.4 \mathrm{mmol}, 74.7 \mu \mathrm{L})$, $\mathrm{TsOH} \cdot \mathrm{H}_{2} \mathrm{O}(76.1 \mathrm{mg}, 0.4 \mathrm{mmol})$ and $\mathrm{Pd}(\mathrm{OAc})_{2}(2.3 \mathrm{mg}, 0.01 \mathrm{mmol})$ were added to a Schlenk tube. Then solvent $(1.5 \mathrm{ml})$ was added by syringe. The reaction mixture was stirred at room temperature for $12 \mathrm{~h}$. Then the reaction mixture was filtered through celite and washed with $\mathrm{CH}_{2} \mathrm{Cl}_{2}$. The mixture was neutralized with saturated $\mathrm{K}_{2} \mathrm{CO}_{3}$ aqueous solution. The aqueous phase was re-extracted with $\mathrm{CH}_{2} \mathrm{Cl}_{2}(3 \times 5 \mathrm{ml})$. The combined organic extracts were dried over $\mathrm{Na}_{2} \mathrm{SO}_{4}$ and the solvent was removed under reduced pressure. 4-Methoxyphenol was added and the mixture was dissolved in $1 \mathrm{ml} \mathrm{CDCl}_{3}$. An aliquot was removed and the yield was determined by ${ }^{1} \mathrm{H}$ NMR spectroscopy. Chemical shifts of methyl groups in 3aa and internal standard were given in Scheme $\mathbf{1}$. 


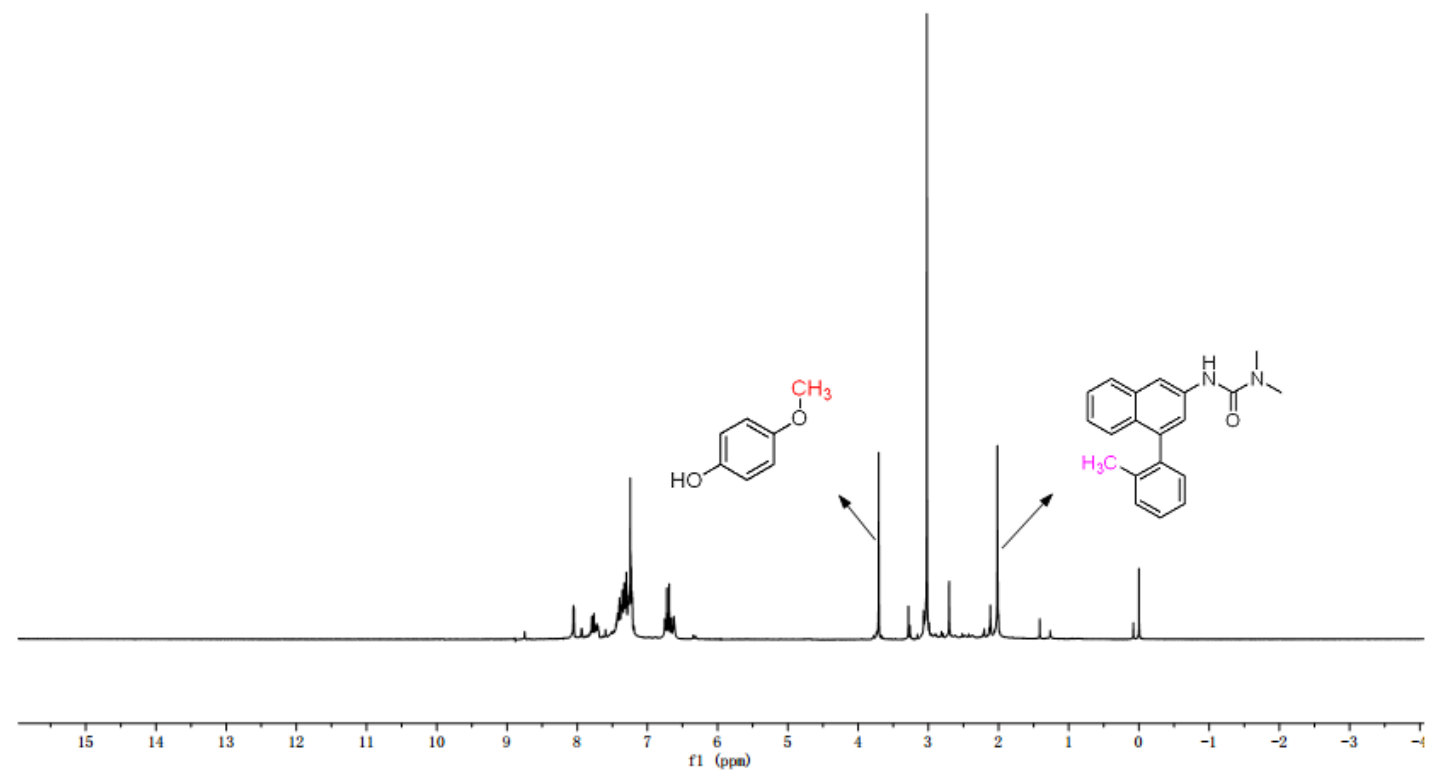

Scheme 1. Chemical shifts of methyl groups in 3aa and internal standard. NMR yields were calculated according to the integration of methyl groups.

\section{(C) General procedure for direct arylation.}

1,1-Dimethyl-3-(naphthalen-2-yl)urea (1a) $(42.8 \mathrm{mg}, 0.2 \mathrm{mmol})$, aryl boronic acid (0.6 mmol), $\mathrm{PhCO}_{3} \mathrm{Bu}-t$ (77.7 mg, $0.4 \mathrm{mmol}, 74.7 \mu \mathrm{L}), \mathrm{Cu}(\mathrm{OAc})_{2} \cdot \mathrm{H}_{2} \mathrm{O}\left(39.9 \mathrm{mg}, 0.2 \mathrm{mmol}\right.$ ), TsOH$\cdot \mathrm{H}_{2} \mathrm{O}(76.1 \mathrm{mg}, 0.4 \mathrm{mmol})$ and $\mathrm{Pd}(\mathrm{OAc})_{2}(2.3 \mathrm{mg}, 0.01 \mathrm{mmol})$ were added to a Schlenk tube under air atmosphere. Then $\mathrm{AcOH}(1.5 \mathrm{ml})$ was added by syringe. The Schlenk tube was capped and the reaction mixture was kept stirring at $25^{\circ} \mathrm{C}$ After the reaction was finished, the resulted mixture was filtrated through celite and washed with $\mathrm{CH}_{2} \mathrm{Cl}_{2}$. The mixture was neutralized with saturated $\mathrm{K}_{2} \mathrm{CO}_{3}$ aqueous solution. The aqueous phase was extracted with $\mathrm{CH}_{2} \mathrm{Cl}_{2}(3 \times 5 \mathrm{ml})$. The combined organic phase were dried over $\mathrm{Na}_{2} \mathrm{SO}_{4}$ and concentrated under reduced pressure. The residue was purified by flash chromatography on silica gel (petroleum ether/dichloromethane as an eluent) to afford the meta-arylated products.

Conditions for direct arylation of indole and pyrrole derivatives (procedures are the same as above):

Condition $\boldsymbol{a}: \mathrm{Pd}(\mathrm{OAc})_{2} 10 \mathrm{~mol} \%, \mathrm{Cu}(\mathrm{OAc})_{2} \cdot \mathrm{H}_{2} \mathrm{O}$ (1.0 eq.), $\mathrm{PhCO}_{3} \mathrm{Bu}$ (2eq.), TsOH$\cdot \mathrm{H}_{2} \mathrm{O}$ (1.0 eq.), $\mathrm{HOAc}$ as solvent $(3 \mathrm{~mL}), 25^{\circ} \mathrm{C}$ Condition $\boldsymbol{b}: \mathrm{Pd}(\mathrm{OAc})_{2} 10 \mathrm{~mol} \%, \mathrm{~K}_{2} \mathrm{~S}_{2} \mathrm{O}_{8}(2.0$ eq. $), \mathrm{TsOH} \cdot \mathrm{H}_{2} \mathrm{O}(1.0$ eq. $), \mathrm{HOAc}:$ toluene $=$ $3 \mathrm{~mL}: 1 \mathrm{~mL}$ as solvent, $25^{\circ} \mathrm{C}$

\section{(D) ${ }^{1} \mathrm{H}$ and ${ }^{13} \mathrm{C}$ NMR data of isolated compounds.}




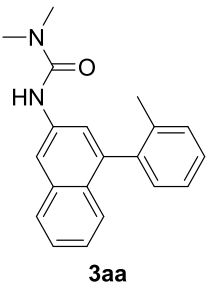

The reaction was performed under general procedure for 6 hours. Flash chromatography (petroleum ether: ethyl acetate $=1: 1)$ yielded the product 3aa $(47.4 \mathrm{mg}, 78 \%)$, white solid, mp.162 ${ }^{\circ} \mathrm{C} ;{ }^{1} \mathrm{H}$ NMR $\left(300 \mathrm{MHz}, \mathrm{cdcl}_{3}\right) \delta(\mathrm{ppm}) 8.07(\mathrm{~d}, J=2.1 \mathrm{~Hz}, 1 \mathrm{H}), 7.79(\mathrm{~d},=7.8 \mathrm{~Hz}, 1 \mathrm{H})$, $7.43-7.26(\mathrm{~m}, 5 \mathrm{H}), 7.24-7.22(\mathrm{~m}, 3 \mathrm{H}), 6.56(\mathrm{br}, 1 \mathrm{H}), 3.03(\mathrm{~s}, 6 \mathrm{H}), 2.02(\mathrm{~s}, 3 \mathrm{H}),{ }^{13} \mathrm{C}$ NMR $(75$ $\left.\mathrm{MHz}, \mathrm{cdcl}_{3}\right) \delta(\mathrm{ppm}) 156.0,140.5,139.8,136.8,136.4,134.4,130.3,129.9,128.7,127.8,127.7,126.2,125.8$, 125.6, 124.5, 121.4, 115.5, 36.5 (2C), 20.1; HRMS (ESI) m/z calcd for $\mathrm{C}_{20} \mathrm{H}_{21} \mathrm{~N}_{2} \mathrm{O}\left([\mathrm{M}+\mathrm{H}]^{+}\right)$: 305.1654, found: 305.1648 .

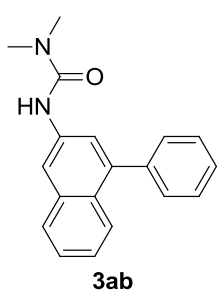

The reaction was performed under general procedure for 12 hours. Flash chromatography (petroleum ether: ethyl acetate $=1: 1)$ yielded the product 3ab $(37.1 \mathrm{mg}, 64 \%)$, White solid, $\operatorname{mp} .177{ }^{0} \mathrm{C} ;{ }^{1} \mathrm{H}$ NMR $\left(300 \mathrm{MHz}, \mathrm{cdcl}_{3}\right) \delta(\mathrm{ppm}) 8.04(\mathrm{~d}, J=2.1 \mathrm{~Hz}, 1 \mathrm{H}), 7.81-7.77(\mathrm{~m}, 2 \mathrm{H})$, $7.48-7.40(\mathrm{~m}, 6 \mathrm{H}), 7.34-7.27(\mathrm{~m}, 2 \mathrm{H}), 6.53(\mathrm{br}, 1 \mathrm{H}), 3.05(\mathrm{~s}, 6 \mathrm{H}) ;{ }^{13} \mathrm{C} \mathrm{NMR}\left(75 \mathrm{MHz}, \mathrm{cdcl}_{3}\right)$ $\delta(\mathrm{ppm}) 156.0,141.0,140.4,136.4,134.7,130.1$ (2C), 128.4, $128.3(2 \mathrm{C}), 127.9,127.4,126.3$, 125.8, 124.6, 121.8, 115.7, 36.5 (2C); HRMS (ESI) m/z calcd for $\mathrm{C}_{19} \mathrm{H}_{19} \mathrm{~N}_{2} \mathrm{O}\left([\mathrm{M}+\mathrm{H}]^{+}\right)$: 291.1497, found: 291.1492.

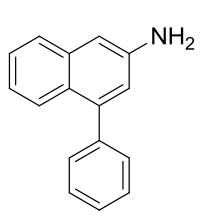

$3 \mathrm{ab}-\mathrm{NH}_{2}$

To a flask was added $3 \mathbf{a b}(29.1 \mathrm{mg}, 0.1 \mathrm{mmol}), \mathrm{NaOH}(40 \mathrm{mg}, 1 \mathrm{mmol})$, EtOH $(2 \mathrm{ml})$, the mixture was refluxed for overnight. After that, the reaction mixture was diluted with $\mathrm{CH}_{2} \mathrm{Cl}_{2}$ through celite and washed with water $(1 \times 5 \mathrm{ml})$. The aqueous phase was re-extracted with $\mathrm{CH}_{2} \mathrm{Cl}_{2}$ $(3 \times 5 \mathrm{ml})$. The combined organic phase were dried over $\mathrm{Na}_{2} \mathrm{SO}_{4}$ and concentrated under reduced pressure, the residue was purified by flash chromatography (petroleum ether: ethyl acetate $=3: 1$ ) to afford the product 3ab- $\mathbf{N H}_{2}(25.9 \mathrm{mg}, 89 \%)$, a pale-yellow oil. ${ }^{1} \mathrm{H} \mathrm{NMR}\left(300 \mathrm{MHz}, \mathrm{cdcl}_{3}\right) \delta(\mathrm{ppm}) 7.70(\mathrm{~d}, J=8.4 \mathrm{~Hz}, 1 \mathrm{H})$, $7.64(\mathrm{~d}, J=8.4 \mathrm{~Hz}, 1 \mathrm{H}), 7.47-7.33(\mathrm{~m}, 6 \mathrm{H}), 7.16(\mathrm{t}, J=7.8 \mathrm{~Hz}, 1 \mathrm{H}), 7.00(\mathrm{~d}, J=2.1 \mathrm{~Hz}, 1 \mathrm{H}), 6.89(\mathrm{~d}, J=2.4 \mathrm{~Hz}$ 1H), 3.84 (br, 2H); ${ }^{13} \mathrm{C}$ NMR (75 MHz, $\left.\mathrm{cdcl}_{3}\right) \delta(\mathrm{ppm}) 143.5,141.6,140.6,135.4,129.9$ (2C), 128.2 (2C), 127.2, 126.3, 126.2, 126.1, 126.0, 122.6, 119.2, 108.3; The NMR data are consistent with that reported in the literature. ${ }^{1}$

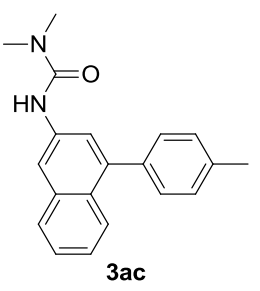

The reaction was performed under general procedure for 6 hours. Flash chromatography (petroleum ether: ethyl acetate=1:1) yielded the product 3ac $(40.1 \mathrm{mg}, 66 \%)$, white solid, mp.138 ${ }^{0} \mathrm{C} ;{ }^{1} \mathrm{H}$ NMR $\left(300 \mathrm{MHz}, \mathrm{cdcl}_{3}\right) \delta(\mathrm{ppm}) 8.05(\mathrm{~d}, J=2.1 \mathrm{~Hz}, 1 \mathrm{H}), 7.82-7.78(\mathrm{~m}, 2 \mathrm{H})$, 
$7.44-7.28(\mathrm{~m}, 7 \mathrm{H}), 6.51(\mathrm{br}, 1 \mathrm{H}), 3.05(\mathrm{~s}, 6 \mathrm{H}), 2.44(\mathrm{~s}, 3 \mathrm{H}) ;{ }^{13} \mathrm{C} \mathrm{NMR}\left(75 \mathrm{MHz}, \mathrm{cdcl}_{3}\right) \delta(\mathrm{ppm})$ 155.7, 141.0, 137.3, 137.0, 136.1, 134.6, 129.8 (2C), 128.9 (2C), 128.4, 127.8, 126.1, 125.8, 124.3, 121.3, 115.3, 36.6 (2C), 21.1; HRMS (ESI) m/z calcd for $\mathrm{C}_{20} \mathrm{H}_{21} \mathrm{~N}_{2} \mathrm{O}\left([\mathrm{M}+\mathrm{H}]^{+}\right): 305.1654$, found: 305.1648 .

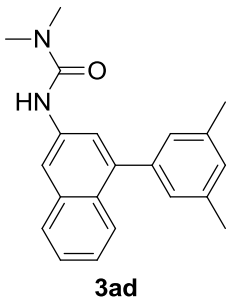

The reaction was performed under general procedure for 12 hours. Flash chromatography (petroleum ether: ethyl acetate=1:1) yielded the product 3ad $(40.7 \mathrm{mg}, 64 \%)$, white solid, mp. $169{ }^{0} \mathrm{C} ;{ }^{1} \mathrm{H}$ NMR $(300 \mathrm{MHz}, \mathrm{dmso}) \delta(\mathrm{ppm}) 8.50$ (br, 1H) 8.08 (s, 1H), $7.80-7.69(\mathrm{~m}, 2 \mathrm{H})$, $7.59-7.58(\mathrm{~m}, 1 \mathrm{H}), 7.45-7.27(\mathrm{~m}, 3 \mathrm{H}), 7.08-7.06(\mathrm{~m}, 2 \mathrm{H}), 2.97(\mathrm{~s}, 6 \mathrm{H}), 2.36(\mathrm{~s}, 6 \mathrm{H}) ;{ }^{13} \mathrm{C}$ NMR (75 MHz, $\left.\mathrm{cdcl}_{3}\right) \delta(\mathrm{ppm})$ 155.9, 141.5, 140.3, 137.8 (2C), 136.3, 134.8, 130.0, 129.1 (2C), 128.6, 128.0, 126.3, 126.1, 124.5, 121.4, 115.4, 36.7 (2C), 21.5 (2C); HRMS (ESI) m/z calcd for $\mathrm{C}_{21} \mathrm{H}_{23} \mathrm{~N}_{2} \mathrm{O}$ $\left([\mathrm{M}+\mathrm{H}]^{+}\right): 319.1810$, found: 319.1805 .

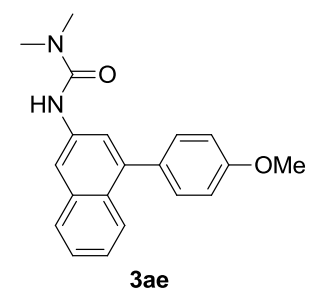

The reaction was performed under general procedure for 3 hours. Flash chromatography petroleum ether: ethyl acetate $=1: 1)$ yielded the product 3ae $(40.3 \mathrm{mg}, 63 \%)$, white solid, mp. $163{ }^{0} \mathrm{C} ;{ }^{1} \mathrm{H}$ NMR $\left(300 \mathrm{MHz}, \mathrm{cdcl}_{3}\right) \delta(\mathrm{ppm}) 8.03(\mathrm{~d}, J=2.1 \mathrm{~Hz}, 1 \mathrm{H}), 7.82-7.78(\mathrm{~m}$, 2H), $7.45-7.39(\mathrm{~m}, 3 \mathrm{H}), 7.32-7.27(\mathrm{~m}, 2 \mathrm{H}) 7.03-6.99(\mathrm{~m}, 2 \mathrm{H}), 6.51(\mathrm{br}, 1 \mathrm{H}), 3.88(\mathrm{~s}$, 3H), $3.06(\mathrm{~s}, 6 \mathrm{H}) ;{ }^{3} \mathrm{C}$ NMR $\left(75 \mathrm{MHz}, \mathrm{cdcl}_{3}\right) \delta(\mathrm{ppm}) 159.1,156.0,140.6,136.4,134.8,132.8,131.1$ (2C), 128.6, 127.9, 126.2, 125.9, 124.4, 121.7, 115.4, 113.8 (2C), 55.4, 36.5 (2C); HRMS (ESI) m/z calcd for $\mathrm{C}_{20} \mathrm{H}_{21} \mathrm{~N}_{2} \mathrm{O}_{2}$ $\left([\mathrm{M}+\mathrm{H}]^{+}\right): 321.1603$, found: 321.1598 .

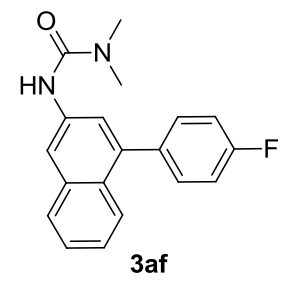

The reaction was performed under general procedure for 5 hours. Flash chromatography (petroleum ether: ethyl acetate=1:1) yielded the product 3af $(37.0 \mathrm{mg}, 60 \%)$, White solid, mp. $158{ }^{0} \mathrm{C} ;{ }^{1} \mathrm{H}$ NMR $\left(300 \mathrm{MHz}, \mathrm{cdcl}_{3}\right) \delta(\mathrm{ppm}) 7.99(\mathrm{~d}, J=1.8 \mathrm{~Hz}, 1 \mathrm{H}), 7.80-7.71(\mathrm{~m}, 2 \mathrm{H})$, $7.45-7.41(\mathrm{~m}, 3 \mathrm{H}), 7.36(\mathrm{~d}, J=2.1 \mathrm{~Hz}, 1 \mathrm{H}), 7.33-7.28(\mathrm{~m}, 1 \mathrm{H}), 7.17-7.12(\mathrm{~m}, 2 \mathrm{H}), 6.56$ (br, 1H), $3.05(\mathrm{~s}, 6 \mathrm{H}),{ }^{13} \mathrm{C}$ NMR $\left(75 \mathrm{MHz}, \mathrm{cdcl}_{3}\right) \delta(\mathrm{ppm}) 162.3\left(\mathrm{~d}, J_{C-F}=245.0 \mathrm{~Hz}\right), 155.7,139.8,136.2\left(\mathrm{~d}, J_{C-F}=\right.$ $3.0 \mathrm{~Hz}), 136.1,134.6,131.5\left(\mathrm{~d}, J_{C-F}=7.5 \mathrm{~Hz}, 2 \mathrm{C}\right), 128.3,127.8,126.2,125.5,124.6,121.6,115.6,115.1\left(\mathrm{~d}, J_{C-F}\right.$ $=21.8 \mathrm{~Hz}, 2 \mathrm{C}), 36.4$ (2C); HRMS (ESI) m/z calcd for $\mathrm{C}_{19} \mathrm{H}_{18} \mathrm{FN}_{2} \mathrm{O}\left([\mathrm{M}+\mathrm{H}]^{+}\right): 309.1403$, found: 309.1398 . 


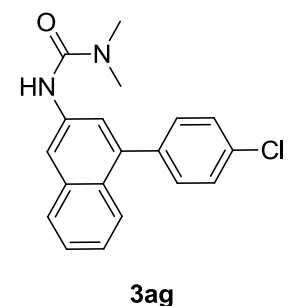

The reaction was performed under general procedure for 12 hours. Flash chromatography (petroleum ether: ethyl acetate $=1: 1)$ yielded the product $\mathbf{3 a g}(33.7 \mathrm{mg}, 52 \%)$, White solid, mp. $178^{0} \mathrm{C} ;{ }^{1} \mathrm{H}$ NMR $\left(300 \mathrm{MHz}, \mathrm{cdcl}_{3}\right) \delta(\mathrm{ppm}) 8.00(\mathrm{~d}, J=1.8 \mathrm{~Hz}, 1 \mathrm{H}), 7.81-7.71(\mathrm{~m}, 2 \mathrm{H})$, $7.45-7.28(\mathrm{~m}, 7 \mathrm{H}), 6.54(\mathrm{br}, 1 \mathrm{H}), 3.06(\mathrm{~s}, 6 \mathrm{H}) ;{ }^{13} \mathrm{C} \mathrm{NMR}(75 \mathrm{MHz}, \mathrm{dmso}) \delta(\mathrm{ppm}) 155.9$, $138.8,138.2,137.9,134.2,132.4,131.4(2 \mathrm{C}), 128.5$ (2C), 127.6, 126.8, 126.2, 124.7, 124.4, 122.0, $114.9,36.3$ (2C); HRMS (ESI) m/z calcd for $\mathrm{C}_{19} \mathrm{H}_{18} \mathrm{ClN}_{2} \mathrm{O}\left([\mathrm{M}+\mathrm{H}]^{+}\right): 325.1108$, found: 325.1102 .

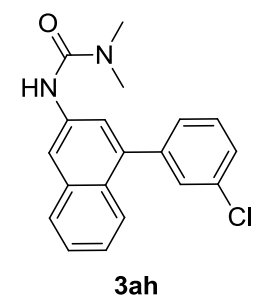

The reaction was performed under general procedure for 12 hours. Flash chromatography (petroleum ether: ethyl acetate=1:1) yielded the product 3ah $(34.4 \mathrm{mg}, 53 \%)$, White solid, mp.159 ${ }^{0} \mathrm{C} ;{ }^{1} \mathrm{H}$ NMR $\left(300 \mathrm{MHz}, \mathrm{cdcl}_{3}\right) \delta(\mathrm{ppm}) 7.96(\mathrm{~d}, J=2.1 \mathrm{~Hz}, 1 \mathrm{H}), 7.75-7.70(\mathrm{~m}, 2 \mathrm{H})$, $7.45-7.26(\mathrm{~m}, 7 \mathrm{H}), 6.75(\mathrm{br}, 1 \mathrm{H}), 2.98(\mathrm{~s}, 6 \mathrm{H}) ;{ }^{13} \mathrm{C} \mathrm{NMR}\left(75 \mathrm{MHz}, \mathrm{cdcl}_{3}\right) \delta(\mathrm{ppm}) 155.8$, $142.1,139.1,136.2,134.4,134.0,129.8,129.3,128.2,127.9,127.7,127.3,126.2,125.2,124.6,121.7,116.1,36.3$ (2C); HRMS (ESI) m/z calcd for $\mathrm{C}_{19} \mathrm{H}_{18} \mathrm{ClN}_{2} \mathrm{O}\left([\mathrm{M}+\mathrm{H}]^{+}\right): 325.1108$, found: 325.1102 .

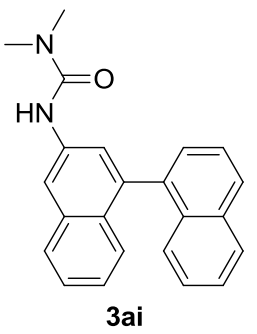

The reaction was performed under general procedure for 5 hours. Flash chromatography (petroleum ether: ethyl acetate $=1: 1$ ) yielded the product 3ai $(47.6 \mathrm{mg}, 70 \%)$, white solid, mp. $216{ }^{0} \mathrm{C} ;{ }^{1} \mathrm{H}$ NMR $\left(300 \mathrm{MHz}, \mathrm{cdcl}_{3}\right) \delta(\mathrm{ppm}) 8.21(\mathrm{~d}, J=1.8 \mathrm{~Hz}, 1 \mathrm{H}), 7.94-7.83(\mathrm{~m}, 3 \mathrm{H})$, $7.58-7.27(\mathrm{~m}, 8 \mathrm{H}), 7.18-7.13(\mathrm{~m}, 1 \mathrm{H}), 6.54(\mathrm{br}, 1 \mathrm{H}), 3.02(\mathrm{~s}, 6 \mathrm{H}) ;{ }^{13} \mathrm{C}$ NMR $(75 \mathrm{MHz}$, dmso) $\delta(\mathrm{ppm}) 155.7,137.9,137.7,137.5,133.9,133.1,132.0,128.2,128.1,127.9,127.5$ (2C), 126.2, 126.1, 125.9, 125.7, 125.5, 125.3, 124.0, 122.6, 114.6, 36.2 (2C); HRMS (ESI) m/z calcd for $\mathrm{C}_{23} \mathrm{H}_{21} \mathrm{~N}_{2} \mathrm{O}$ $\left([\mathrm{M}+\mathrm{H}]^{+}\right): 341.1654$, found:341.1648.

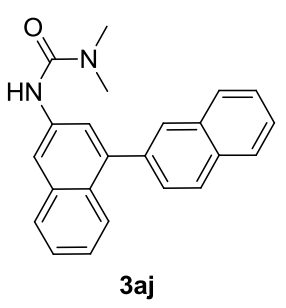

The reaction was performed under general procedure for 12 hours. Flash chromatography (petroleum ether: ethyl acetate=1:1) yielded the product 3aj (37.4 mg, $55 \%$ ), White solid, mp.93 ${ }^{0} \mathrm{C} ;{ }^{1} \mathrm{H}$ NMR (300 MHz, dmso) $\delta(\mathrm{ppm}) 8.58(\mathrm{br}, 1 \mathrm{H}), 8.15(\mathrm{~s}, 1 \mathrm{H}), 8.08-8.00(\mathrm{~m}$, 4H), $7.84(\mathrm{~d}, J=8.1 \mathrm{~Hz}, 1 \mathrm{H}), 7.74-7.72(\mathrm{~m}, 2 \mathrm{H}), 7.65-7.57(\mathrm{~m}, 3 \mathrm{H}), 7.46(\mathrm{t}, J=7.2 \mathrm{~Hz}$, 1H), $7.31(\mathrm{t}, J=7.2 \mathrm{~Hz}, 1 \mathrm{H}), 2.98(\mathrm{~s}, 6 \mathrm{H}) ;{ }^{13} \mathrm{C}$ NMR $(75 \mathrm{MHz}, \mathrm{dmso}) \delta(\mathrm{ppm})$ 155.8, 139.4, 138.0, 137.5, 134.2, 133.0, 132.1, 128.2, 128.0, 127.9, 127.7, 127.6 (2C), 127.0, 126.5, 126.2, 126.1, 125.0, 124.2, 122.3, 114.6, 36.3 (2C); HRMS (ESI) m/z calcd for $\mathrm{C}_{23} \mathrm{H}_{21} \mathrm{~N}_{2} \mathrm{O}\left([\mathrm{M}+\mathrm{H}]^{+}\right): 341.1654$, found: 341.1627 . 


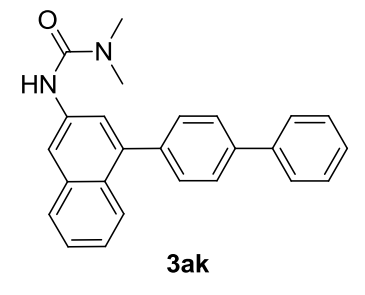

The reaction was performed under general procedure using $0.3 \mathrm{mmol} 4$-Biphenylboronic acid (1.5 eq., $59.4 \mathrm{mg}$ ) for 12 hours. Flash chromatography (petroleum ether: ethyl acetate $=1: 1)$ yielded the product 3ak $(57.1 \mathrm{mg}, 78 \%)$, White solid, $\mathrm{mp} .>250^{\circ} \mathrm{C}$, decomposed; ${ }^{1} \mathrm{H}$ NMR (300 MHz, dmso) $\delta$ (ppm) 8.54 (br, 1H), 8.12 (s, 1H), $7.84-7.75$ (m, 6H), $7.69(\mathrm{~d}, J=1.8 \mathrm{~Hz}, 1 \mathrm{H}), 7.58-7.31(\mathrm{~m}, 8 \mathrm{H}), 2.98(\mathrm{~s}, 6 \mathrm{H}),{ }^{13} \mathrm{C}$ NMR $(75 \mathrm{MHz}, \mathrm{dmso}) \delta(\mathrm{ppm})$ 155.7, $139.7,139.2,139.0,138.0,134.2,130.2,129.0,127.6,127.5,126.8,126.7,126.1,124.9,124.2,121.9,114.5,36.2$ (2C); HRMS (ESI) $\mathrm{m} / \mathrm{z}$ calcd for $\mathrm{C}_{25} \mathrm{H}_{23} \mathrm{~N}_{2} \mathrm{O}\left([\mathrm{M}+\mathrm{H}]^{+}\right): 366.1810$, found: 367.1805

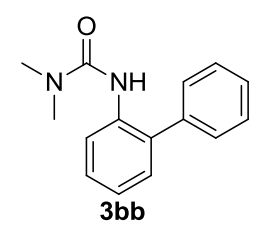

The reaction was performed under general procedure using $1 \mathrm{mmol} \mathbf{1 b}(164.1 \mathrm{mg}), 10 \mathrm{~mol} \%$ $\mathrm{Pd}(\mathrm{OAc})_{2}, 0.5$ eq. $\mathrm{TsOH} \cdot \mathrm{H}_{2} \mathrm{O}$ and $3 \mathrm{~mL}$ acetic acid for 48 hours. Flash chromatography (petroleum ether: ethyl acetate $=1: 1)$, yielded the product $\mathbf{3 b b}$ as white solid $(183.0 \mathrm{mg}, 76 \%$ );

${ }^{1} \mathrm{H}$ NMR $\left(300 \mathrm{MHz}, \mathrm{cdcl}_{3}\right) \delta(\mathrm{ppm}) 8.19(\mathrm{dd}, J=1.2$ and $8.2 \mathrm{~Hz}, 1 \mathrm{H}), 7.50-7.31(\mathrm{~m}, 6 \mathrm{H}), 7.19(\mathrm{dd}, J=1.5$ and $7.6 \mathrm{~Hz}, 1 \mathrm{H}), 7.07$ (dt, $J=1.2$ and $7.5 \mathrm{~Hz}, 1 \mathrm{H}), 6.50$ (brs, $1 \mathrm{H}), 2.80(\mathrm{~s}, 6 \mathrm{H}) ;{ }^{13} \mathrm{C} \mathrm{NMR}\left(75 \mathrm{MHz}, \mathrm{cdcl}_{3}\right) \delta(\mathrm{ppm})$ $155.6,138.8,136.4,131.5,129.7,129.4(2 \mathrm{C}), 129.2(2 \mathrm{C}), 128.5,128.0,122.7,120.5,36.3$. The NMR data are consistent with that reported in the literature. ${ }^{2}$

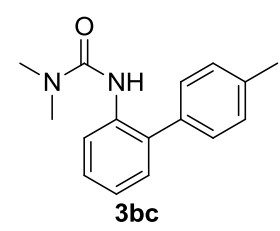

The reaction was performed under general procedure using $1 \mathrm{mmol} \mathbf{1 b}(164.1 \mathrm{mg}), 10 \mathrm{~mol} \%$ $\mathrm{Pd}(\mathrm{OAc})_{2}, 0.5$ eq. TsOH$\cdot \mathrm{H}_{2} \mathrm{O}$ and $3 \mathrm{~mL}$ acetic acid for 48 hours. Flash chromatography (petroleum ether: ethyl acetate $=1: 1$ ) yielded the product $3 \mathbf{b c}$ as white solid, (in HOAc : 62.3 mg, $25 \%$ ); (in PhMe : 146.1mg, $57 \%$ ). ${ }^{1} \mathrm{H}$ NMR (300 MHz, $\left.\mathrm{cdcl}_{3}\right) \delta$ (ppm) 8.18 (d, $J=8.4$ $\mathrm{Hz}, 1 \mathrm{H}), 7.35-7.28(\mathrm{~m}, 5 \mathrm{H}), 7.18(\mathrm{~d}, J=7.5 \mathrm{~Hz}, 1 \mathrm{H}), 7.06$ (t, $J=7.4 \mathrm{~Hz}, 1 \mathrm{H}), 6.56$ (brs, 1H), 2.81 (s, 6H), 2.41 (s, 3H). $\left.{ }^{13} \mathrm{C} \mathrm{NMR} \mathrm{(75} \mathrm{MHz,} \mathrm{cdcl}_{3}\right) \delta(\mathrm{ppm})$ 155.6, 137.7, 136.5, 135.8, 131.4, 129.9 (2C), 129.8, 129.3 (2C), 128.4, 122.6, 120.4, 36.3, 21.4. The NMR data are consistent with that reported in the literature. ${ }^{2}$

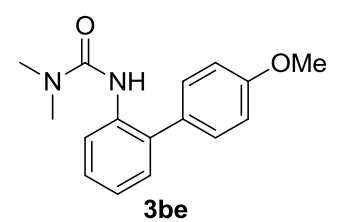

The reaction was performed under general procedure using $1 \mathrm{mmol} \mathbf{1 b}(164.1 \mathrm{mg}), 10 \mathrm{~mol}$ $\% \mathrm{Pd}(\mathrm{OAc})_{2}, 0.5$ eq. Ts $\mathrm{OH} \cdot \mathrm{H}_{2} \mathrm{O}$ and $3 \mathrm{~mL}$ acetic acid for 48 hours. Flash chromatography (petroleum ether : ethyl acetate $=1: 1$ ) yielded the product 3be as white solid, (in HOAc : $60.3 \mathrm{mg}, 22 \%$ ), (in PhMe : $168.6 \mathrm{mg}, 62 \%) .{ }^{1} \mathrm{H}$ NMR (300 MHz, cdcl 3 ) $\delta(\mathrm{ppm}) 8.17$ (d, $J=8.1 \mathrm{~Hz}, 1 \mathrm{H}), 7.35-7.29(\mathrm{~m}, 3 \mathrm{H}), 7.17(\mathrm{dd}, J=1.8$ and $7.5 \mathrm{~Hz}, 1 \mathrm{H}), 7.08-6.99(\mathrm{~m}, 3 \mathrm{H}), 6.53$ (brs, $1 \mathrm{H}), 3.86$ (s, 3H), $2.82(\mathrm{~s}, 6 \mathrm{H}) ;{ }^{13} \mathrm{C}$ NMR (75 MHz, $\left.\mathrm{cdcl}_{3}\right) . \delta(\mathrm{ppm}) 159.4,155.6,136.7,131.1,130.9,130.6$ (2C), 129.9, 
128.3, 122.6, 120.4, $114.6(2 \mathrm{C}), 55.5,36.4$. The NMR data are consistent with that reported in the literature. ${ }^{3}$

The reaction was performed under general procedure using $1 \mathrm{mmol} \mathbf{1 b}(164.1 \mathrm{mg}), 10 \mathrm{~mol}$<smiles>CN(C)C(=O)Nc1ccccc1-c1ccc(F)cc1</smiles>
$\% \mathrm{Pd}(\mathrm{OAc})_{2}, 0.5$ eq. Ts $\mathrm{OH} \cdot \mathrm{H}_{2} \mathrm{O}$ and $3 \mathrm{~mL}$ acetic acid for 24 hours. Flash chromatography (petroleum ether: ethyl acetate $=1: 1)$ yielded the product $\mathbf{3 b f}$ as white solid $(207.1 \mathrm{mg}, 80$ \%). ${ }^{1} \mathrm{H}$ NMR (300 MHz, $\left.\mathrm{cdcl}_{3}\right) \delta(\mathrm{ppm}) 8.12(\mathrm{~d}, J=8.1 \mathrm{~Hz}, 1 \mathrm{H}), 7.37-7.30(\mathrm{~m}, 3 \mathrm{H}), 7.19$ -7.04 (m, 3H), 6.38 (brs, 1H), 2.81 (s, 6H); $\left.{ }^{13} \mathrm{C} \mathrm{NMR} \mathrm{(75} \mathrm{MHz,} \mathrm{cdcl}_{3}\right) \delta(\mathrm{ppm}) 162.4(\mathrm{~d}$, $\left.J_{C F}=246.0 \mathrm{~Hz}\right), 155.5,136.4,134.7,131.1\left(\mathrm{~d}, J_{C F}=7.5 \mathrm{~Hz}\right), 130.7,129.8,128.7,122.9,121.0,116.1\left(\mathrm{~d}, J_{C F}=21.0\right.$ $\mathrm{Hz}$ ), 36.3. The NMR data are consistent with that reported in the literature. ${ }^{4}$

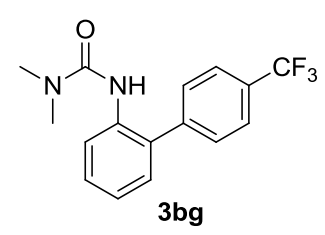

The reaction was performed under general procedure using $1 \mathrm{mmol} \mathbf{1 b}(164.1 \mathrm{mg}), 10$ mol $\% \mathrm{Pd}(\mathrm{OAc})_{2}, 0.5$ eq. $\mathrm{TsOH} \cdot \mathrm{H}_{2} \mathrm{O}$ and $3 \mathrm{~mL}$ acetic acid for 48 hours. Flash chromatography (petroleum ether : ethyl acetate $=1: 1$ ) yielded the product $3 \mathbf{b g}$ as white solid, mp : $185{ }^{\circ} \mathrm{C},(184.0 \mathrm{mg}, 60 \%) .{ }^{1} \mathrm{H}$ NMR $\left(300 \mathrm{MHz}, \mathrm{cdcl}_{3}\right) \delta(\mathrm{ppm}) 8.09$ (d, $J=8.4$ $\mathrm{Hz}, 1 \mathrm{H}), 7.74(\mathrm{~d}, J=8.1 \mathrm{~Hz}, 2 \mathrm{H}), 7.54(\mathrm{~d}, J=8.1 \mathrm{~Hz}, 2 \mathrm{H}), 7.41-7.35(\mathrm{~m}, 1 \mathrm{H}), 7.21-7.10(\mathrm{~m}, 2 \mathrm{H}), 6.28$ (brs, 1H), $2.83(\mathrm{~s}, 6 \mathrm{H}) ;{ }^{13} \mathrm{C}$ NMR (75 MHz, $\left.\mathrm{cdcl}_{3}\right) \delta(\mathrm{ppm})$ 155.6, 142.8, 136.2, 130.8 (2C), 129.9, 129.8, 129.2, 126.0 $\left(\mathrm{q}, J_{C F}=3.7 \mathrm{~Hz}, 2 \mathrm{C}\right), 123.4,122.0,36.4\left(\mathrm{CF}_{3}\right.$ and $C-\mathrm{CF}_{3}$ are partially overlapped with other peaks). HRMS (ESI) $\mathrm{m} / \mathrm{z}$ calcd for $\mathrm{C}_{16} \mathrm{H}_{16} \mathrm{~F}_{3} \mathrm{~N}_{2} \mathrm{O}\left([\mathrm{M}+\mathrm{H}]^{+}\right): 309.1215$, found : 309.1209 .

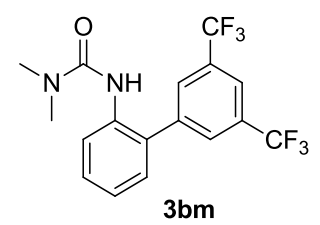

The reaction was performed under general procedure using $1 \mathrm{mmol} \mathbf{1 b}(164.1 \mathrm{mg}), 10$ mol $\% \mathrm{Pd}(\mathrm{OAc})_{2}, 0.5$ eq. $\mathrm{TsOH} \cdot \mathrm{H}_{2} \mathrm{O}$ and $3 \mathrm{~mL}$ acetic acid for 48 hours. Flash chromatography (petroleum ether : ethyl acetate $=1: 1$ ) yielded the product $\mathbf{3 b m}$ as white solid (241.2 mg, $64 \%) .{ }^{1} \mathrm{H}$ NMR (300 MHz, $\left.\mathrm{cdcl}_{3}\right) \delta(\mathrm{ppm}) 8.00-7.91(\mathrm{~m}, 4 \mathrm{H}), 7.45-$ $7.39(\mathrm{~m}, 1 \mathrm{H}), 7.25-7.17$ (m, 2H), 6.14 (brs, 1H), 2.85 (s, 6H); $\left.{ }^{13} \mathrm{C} \mathrm{NMR} \mathrm{(75} \mathrm{MHz,} \mathrm{cdcl}_{3}\right) \delta(\mathrm{ppm})$ 155.7, 141.5, 136.1, 132.6, 132.1, 130.6, 129.9, 129.7, 125.2, 124.5, 123.9, 121.4, 36.3. The NMR data are consistent with that reported in the literature. ${ }^{4}$

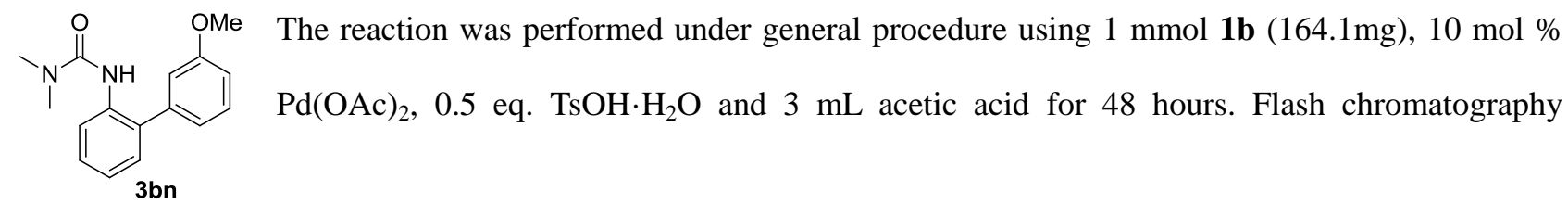


(petroleum ether : ethyl acetate $=1: 1$ ), yielded the product 3bn as white solid, mp: $98{ }^{\circ} \mathrm{C}$, (in HOAc : $29.7 \mathrm{mg}$, $11 \%$ ), (in PhMe : $140.2 \mathrm{mg}, 52 \%) .{ }^{1} \mathrm{H}$ NMR $\left(300 \mathrm{MHz}, \mathrm{cdcl}_{3}\right) \delta$ (ppm) 8.19 (d, $\left.J=8.1 \mathrm{~Hz}, 1 \mathrm{H}\right), 7.40-7.31$ (m, $2 \mathrm{H}), 7.20$ (d, $J=7.2 \mathrm{~Hz}, 1 \mathrm{H}), 7.06$ (t, $J=7.2 \mathrm{~Hz}, 1 \mathrm{H}), 6.98-6.92$ (m, 3H), 6.61 (brs, 1H), 3.83 (s, 3H), 2.81 (s, $6 \mathrm{H}) ;{ }^{13} \mathrm{C}$ NMR $\left(75 \mathrm{MHz}, \mathrm{cdcl}_{3}\right) \delta(\mathrm{ppm}) 160.3,155.7,140.2,136.5,131.3,130.2,129.6,128.7,122.6,121.5$, 120.4, 114.9, 113.7, 55.5, 36.3. HRMS (ESI) m/z calcd for $\mathrm{C}_{16} \mathrm{H}_{19} \mathrm{~N}_{2} \mathrm{O}_{2}\left([\mathrm{M}+\mathrm{H}]^{+}\right): 271.1447$; found : 271.1441 .

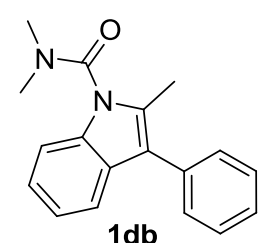

The reaction was performed under condition $\boldsymbol{a}$ and $\boldsymbol{b}$ using $1 \mathrm{mmol} \mathbf{1 d}(202.1 \mathrm{mg})$ for 16 hours. Flash chromatography (petroleum ether : ethyl acetate $=3: 1$ ) yielded the product $\mathbf{1 d b}$ as colorless oil. (condition $\boldsymbol{a}: 91.8 \mathrm{mg}, 33 \%$, condition $\boldsymbol{b}: 172.4 \mathrm{mg}, 62 \%) ;{ }^{1} \mathrm{H}$ NMR (300 MHz, $\left.\mathrm{cdcl}_{3}\right) \delta(\mathrm{ppm}) 7.62-7.59(\mathrm{~m}, 1 \mathrm{H}), 7.51-7.44(\mathrm{~m}, 4 \mathrm{H}), 7.36-7.31(\mathrm{~m}, 1 \mathrm{H}), 7.25-7.20(\mathrm{~m}$, 2H), $7.18-7.12(\mathrm{~m}, 1 \mathrm{H}), 3.09$ (s, 6H), 2.49 (s, 3H); $\left.{ }^{13} \mathrm{C} \mathrm{NMR} \mathrm{(75} \mathrm{MHz,} \mathrm{cdcl}_{3}\right) \delta(\mathrm{ppm})$ 154.5, 135.0, 134.6, 132.4, 129.9 (2C), 128.7 (2C), 128.3, 126.6, 122.9, 121.5, 119.4, 118.0, 111.0, 38.0 (2C), 11.9. HRMS (ESI) m/z for $\mathrm{C}_{18} \mathrm{H}_{19} \mathrm{~N}_{2} \mathrm{O}\left([\mathrm{M}+\mathrm{H}]^{+}\right): 279.1497$, found : 279.1492 .

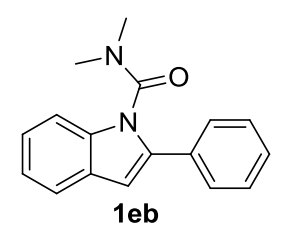

The reaction was performed under condition $\boldsymbol{a}$ and $\boldsymbol{b}$ using $1 \mathrm{mmol} \mathbf{1 e}(188.1 \mathrm{mg})$ for 48 hours. Flash chromatography (petroleum ether : ethyl acetate $=3: 1$ ) yielded the product $\mathbf{1 e b}$ as colorless oil; (condition $\boldsymbol{a}: 110.9 \mathrm{mg}, 42 \%$, condition $\boldsymbol{b}: 200.8 \mathrm{mg}, 72 \%)$. ${ }^{1} \mathrm{H}$ NMR (300 $\left.\mathrm{MHz}, \mathrm{cdcl}_{3}\right) \delta(\mathrm{ppm}) 7.62(\mathrm{~d}, J=7.5 \mathrm{~Hz}, 1 \mathrm{H}), 7.56-7.49(\mathrm{~m}, 3 \mathrm{H}), 7.45-7.33(\mathrm{~m}, 3 \mathrm{H}), 7.30$ $-7.24(\mathrm{~m}, 1 \mathrm{H}), 7.22-7.16(\mathrm{~m}, 1 \mathrm{H}), 6.72(\mathrm{~s}, 1 \mathrm{H}), 3.03$ (brs, 3H), 2.47 (brs, 3H); ${ }^{13} \mathrm{C}$ NMR $\left(75 \mathrm{MHz}, \mathrm{cdcl}_{3}\right) \delta$ (ppm) 154.2, 139.4, 136.9, 132.3, 128.7 (2C), 128.6, 128.1, 127.3 (2C), 123.4, 121.7, 120.8, 111.6, 104.4, 37.7, 36.6. The NMR data are consistent with that reported in the literature. ${ }^{5}$

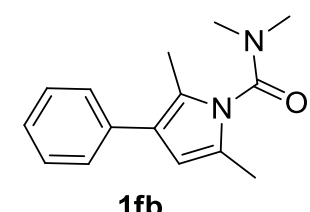

$1 \mathrm{fb}$

The reaction was performed under condition $\boldsymbol{a}$ and $\boldsymbol{b}$ using $1 \mathrm{mmol} \mathbf{1 f}(166.1 \mathrm{mg})$ for 16 hours. Flash chromatography (petroleum ether : ethyl acetate $=3: 1$ ) yielded the product 1fb as colorless oil. (condition $\boldsymbol{b}: 57.7 \mathrm{mg}, 24 \%) ;{ }^{1} \mathrm{H}$ NMR $\left(300 \mathrm{MHz}, \mathrm{cdcl}_{3}\right) \delta(\mathrm{ppm}) 7.40$ - $7.32(\mathrm{~m}, 4 \mathrm{H}), 7.23-7.17$ (m,1H), 6.05 (s, 1H), 3.17 (brs, 3H), 2.77 (brs, 3H), 2.30 (s, 3H),

2.22 (s, 3H); ${ }^{13} \mathrm{C}$ NMR (75 MHz, $\left.\mathrm{cdcl}_{3}\right) \delta(\mathrm{ppm}) 154.8,136.7,128.5$ (2C), 128.0 (2C), 126.4, 125.6, 122.7, 122.4, 108.2, 37.8, 36.6, 12.1, 11.4. HRMS (ESI) $\mathrm{m} / \mathrm{z}$ calcd for $\mathrm{C}_{15} \mathrm{H}_{19} \mathrm{~N}_{2} \mathrm{O}\left([\mathrm{M}+\mathrm{H}]^{+}\right)$: 243.1497, found : 243.1492 . 


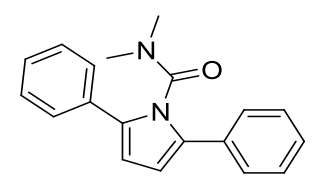

$19 b$

The reaction was performed under condition $\boldsymbol{a}$ and $\boldsymbol{b}$ using $1 \mathrm{mmol} \mathbf{1 g}(138.1 \mathrm{mg})$ for 48 hours. Flash chromatography (petroleum ether : ethyl acetate $=3: 1$ ) yielded the product 1gb as colorless oil; (condition $\boldsymbol{a}: 34.8 \mathrm{mg}, 16 \%$, condition $\boldsymbol{b}: 17.4 \mathrm{mg}, 6 \%$ ). ${ }^{1} \mathrm{H} \mathrm{NMR}$ $\left(300 \mathrm{MHz}, \mathrm{cdcl}_{3}\right) \delta(\mathrm{ppm}) 7.54-7.51(\mathrm{~m}, 4 \mathrm{H}), 7.40-7.35(\mathrm{~m}, 4 \mathrm{H}), 7.31-7.28(\mathrm{~m}, 2 \mathrm{H})$, $6.42(\mathrm{~s}, 2 \mathrm{H}), 2.86(\mathrm{~s}, 3 \mathrm{H}), 2.30(\mathrm{~s}, 3 \mathrm{H}) ;{ }^{13} \mathrm{C}$ NMR (75 MHz, $\left.\mathrm{cdcl}_{3}\right) \delta(\mathrm{ppm}) 154.7,134.9(2 \mathrm{C}), 132.5(2 \mathrm{C}), 128.7$ (4C), 127.3 (6C), 110.1 (2C), 37.2, 36.8. HRMS (ESI) m/z calcd for $\mathrm{C}_{19} \mathrm{H}_{19} \mathrm{~N}_{2} \mathrm{O}\left([\mathrm{M}+\mathrm{H}]^{+}\right)$: 291.1497, found : 291.1492.

\section{(E) Crystallographic data for 3ah (CCDC 1420217).}

Single crystal of 3ah was obtained by recrystallization in $\mathrm{CH}_{2} \mathrm{Cl}_{2}$ and petroleum $(4: 1)$ at room temperature. X-ray structural analysis was performed on a Bruker SMART CCD diffractometer at 293(2) K.

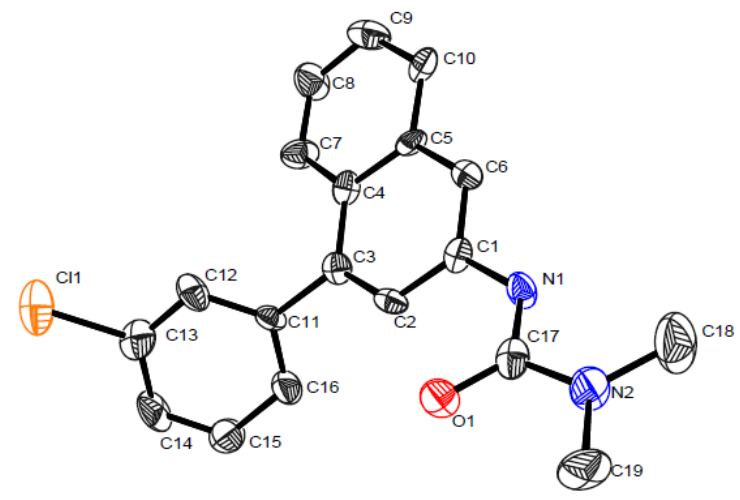

Figure 1. ORTEP plot of compound 3ah. All $\mathrm{H}$ atoms have been omitted for clarity. Anisotropic displacement ellipsoids are shown at the $30 \%$ probability level.

Table 1. Crystal data and structure refinement for3ah.

Identification code

Empirical formula

Formula weight

Temperature

Wavelength

Crystal system, space group

Unit cell dimensions shelx

\section{C38 H34 Cl2 N4 O2.85}

663.24

293(2) K

$0.71073 \mathrm{~A}$

Monoclinic, P 21/c

$$
\begin{gathered}
a=13.0804(14) \text { A } \quad \text { alpha }=90 \text { deg. } \\
b=18.104(2) \text { A } \quad \text { beta }=102.512(3) \mathrm{deg} . \\
c=15.8995(17) \text { A } \quad \text { gamma }=90 \mathrm{deg} .
\end{gathered}
$$


Volume

Z, Calculated density

Absorption coefficient

$\mathrm{F}(000)$

Crystal size

Theta range for data collection

Limiting indices

Reflections collected / unique

Completeness to theta $=25.000$

Refinement method

Data / restraints / parameters

Goodness-of-fit on $\mathrm{F}^{\wedge} 2$

Final R indices [I $>2 \operatorname{sigma}(\mathrm{I})]$

$\mathrm{R}$ indices (all data)

Extinction coefficient

Largest diff. peak and hole
3675.7(7) $\mathrm{A}^{\wedge} 3$

4, $1.199 \mathrm{Mg} / \mathrm{m}^{\wedge} 3$

$0.216 \mathrm{~mm}^{\wedge}-1$

1387

$0.500 \times 0.300 \times 0.020 \mathrm{~mm}$

1.595 to $25.000 \mathrm{deg}$.

$-15<=\mathrm{h}<=15,-20<=\mathrm{k}<=21,-18<=\mathrm{l}<=18$

$23472 / 6485[\mathrm{R}($ int $)=0.1998]$

$100.0 \%$

Full-matrix least-squares on $\mathrm{F}^{\wedge} 2$

$6485 / 6 / 427$

0.918

$$
\begin{aligned}
& \mathrm{R} 1=0.0910, \mathrm{wR} 2=0.2799 \\
& \mathrm{R} 1=0.2165, \mathrm{wR} 2=0.3290 \\
& \mathrm{n} / \mathrm{a} \\
& 1.010 \text { and }-0.313 \mathrm{e} . \mathrm{A}^{\wedge}-3
\end{aligned}
$$

Table 2. Atomic coordinates $\left(\times 10^{\wedge} 4\right)$ and equivalent isotropic displacement parameters $\left(\mathrm{A}^{\wedge} 2 \times 10^{\wedge} 3\right)$ for $3 \mathrm{ah}$. $\mathrm{U}(\mathrm{eq})$ is defined as one third of the trace of the orthogonalized Uij tensor.

\begin{tabular}{ccccc}
\hline & $\mathrm{x}$ & $\mathrm{y}$ & $\mathrm{z}$ & $\mathrm{U}(\mathrm{eq})$ \\
$\mathrm{C}(1)$ & $5754(6)$ & $7797(4)$ & $8795(4)$ & $41(2)$ \\
$\mathrm{C}(2)$ & $6526(6)$ & $8272(4)$ & $8600(4)$ & $42(2)$ \\
$\mathrm{C}(3)$ & $7283(6)$ & $8579(4)$ & $9236(4)$ & $42(2)$ \\
$\mathrm{C}(4)$ & $7315(5)$ & $8406(4)$ & $10121(4)$ & $40(2)$ \\
$\mathrm{C}(5)$ & $6529(6)$ & $7930(4)$ & $10298(4)$ & $41(2)$ \\
$\mathrm{C}(6)$ & $5769(6)$ & $7635(4)$ & $9634(4)$ & $43(2)$ \\
$\mathrm{C}(7)$ & $8093(6)$ & $8667(4)$ & $10817(5)$ & $54(2)$ \\
$\mathrm{C}(8)$ & $8057(7)$ & $8496(5)$ & $11641(5)$ & $65(2)$
\end{tabular}




\begin{tabular}{|c|c|c|c|c|}
\hline $\mathrm{C}(9)$ & $7288(8)$ & $8045(5)$ & $11818(5)$ & $70(3)$ \\
\hline $\mathrm{C}(10)$ & $6540(7)$ & $7755(4)$ & $11170(5)$ & $56(2)$ \\
\hline $\mathrm{C}(11)$ & $8054(5)$ & 9105(4) & 8994(4) & $42(2)$ \\
\hline $\mathrm{C}(12)$ & $8189(6)$ & 9802(4) & $9326(5)$ & $52(2)$ \\
\hline $\mathrm{C}(13)$ & $8870(7)$ & $10280(5)$ & $9069(6)$ & $63(2)$ \\
\hline$C(14)$ & $9420(7)$ & $10070(5)$ & $8449(6)$ & $77(3)$ \\
\hline$C(15)$ & 9297(7) & $9378(5)$ & $8115(6)$ & $74(3)$ \\
\hline$C(16)$ & $8613(6)$ & $8893(5)$ & $8385(5)$ & $57(2)$ \\
\hline $\mathrm{C}(17)$ & $4580(6)$ & $7746(5)$ & $7343(5)$ & $48(2)$ \\
\hline $\mathrm{C}(18)$ & $3440(10)$ & $6625(6)$ & 7091(7) & $118(4)$ \\
\hline$C(19)$ & $3371(8)$ & $7562(5)$ & $5960(5)$ & $83(3)$ \\
\hline$C(20)$ & $3656(6)$ & 10153(4) & 6264(4) & $42(2)$ \\
\hline$C(21)$ & $3045(6)$ & 9573(4) & $6516(5)$ & $50(2)$ \\
\hline$C(22)$ & 2003(6) & $9668(4)$ & $6509(5)$ & $46(2)$ \\
\hline $\mathrm{C}(23)$ & $1512(6)$ & $10356(4)$ & 6273(4) & $40(2)$ \\
\hline$C(24)$ & $2128(6)$ & $10927(4)$ & $6035(4)$ & $43(2)$ \\
\hline$C(25)$ & $3197(6)$ & $10815(4)$ & $6029(5)$ & $48(2)$ \\
\hline$C(26)$ & $461(6)$ & $10508(4)$ & 6286(4) & $48(2)$ \\
\hline $\mathrm{C}(27)$ & $47(6)$ & 11181(5) & $6090(5)$ & $54(2)$ \\
\hline$C(28)$ & $628(6)$ & $11760(5)$ & $5851(5)$ & $54(2)$ \\
\hline $\mathrm{C}(29)$ & $1660(6)$ & 11633(4) & 5815(4) & $48(2)$ \\
\hline $\mathrm{C}(30)$ & 1462(6) & 9008(4) & $6777(5)$ & $50(2)$ \\
\hline $\mathrm{C}(31)$ & 1882(6) & $8659(4)$ & $7540(5)$ & $58(2)$ \\
\hline $\mathrm{C}(32)$ & $1415(7)$ & $8037(5)$ & $7787(5)$ & $65(2)$ \\
\hline $\mathrm{C}(33)$ & $526(7)$ & $7736(5)$ & 7271(6) & $71(3)$ \\
\hline $\mathrm{C}(34)$ & $105(7)$ & $8080(5)$ & $6516(6)$ & $70(3)$ \\
\hline $\mathrm{C}(35)$ & $541(6)$ & $8718(4)$ & $6261(5)$ & $57(2)$ \\
\hline $\mathrm{C}(36)$ & $5465(6)$ & $10407(5)$ & $6086(5)$ & $47(2)$ \\
\hline$C(37)$ & $6585(7)$ & 9276(5) & 6211(6) & $78(3)$ \\
\hline $\mathrm{C}(38)$ & $7282(6)$ & $10492(4)$ & $5943(6)$ & $69(3)$ \\
\hline
\end{tabular}




$\begin{array}{lrrrr}\mathrm{N}(1) & 4988(5) & 7457(3) & 8148(4) & 51(2) \\ \mathrm{N}(2) & 3838(6) & 7309(4) & 6831(4) & 72(2) \\ \mathrm{N}(3) & 4708(5) & 9958(3) & 6281(4) & 54(2) \\ \mathrm{N}(4) & 6400(5) & 10058(4) & 6080(4) & 55(2) \\ \mathrm{O}(1) & 4848(4) & 8337(3) & 7090(3) & 56(1) \\ \mathrm{O}(2) & 5338(4) & 11063(3) & 5922(3) & 57(2) \\ \mathrm{Cl}(1) & 9063(2) & 11162(1) & 9516(2) & 94(1) \\ \mathrm{Cl}(2) & 1981(2) & 7614(2) & 8767(2) & 109(1) \\ \mathrm{O}(3) & 3952(16) & 9341(11) & 9523(12) & 159(7) \\ \mathrm{O}(4) & 4560(30) & 10125(16) & 8800(20) & 204(13)\end{array}$

Table 3. Bond lengths [A] and angles [deg] for 3ah.

\begin{tabular}{ll}
\hline $\mathrm{C}(1)-\mathrm{C}(6)$ & $1.362(9)$ \\
$\mathrm{C}(1)-\mathrm{C}(2)$ & $1.410(9)$ \\
$\mathrm{C}(1)-\mathrm{N}(1)$ & $1.414(9)$ \\
$\mathrm{C}(2)-\mathrm{C}(3)$ & $1.372(9)$ \\
$\mathrm{C}(2)-\mathrm{H}(2)$ & 0.9300 \\
$\mathrm{C}(3)-\mathrm{C}(4)$ & $1.434(9)$ \\
$\mathrm{C}(3)-\mathrm{C}(11)$ & $1.496(9)$ \\
$\mathrm{C}(4)-\mathrm{C}(7)$ & $1.412(10)$ \\
$\mathrm{C}(4)-\mathrm{C}(5)$ & $1.416(9)$ \\
$\mathrm{C}(5)-\mathrm{C}(6)$ & $1.389(9)$ \\
$\mathrm{C}(5)-\mathrm{C}(10)$ & $1.420(9)$ \\
$\mathrm{C}(6)-\mathrm{H}(6)$ & 0.9300 \\
$\mathrm{C}(7)-\mathrm{C}(8)$ & $1.356(10)$ \\
$\mathrm{C}(7)-\mathrm{H}(7)$ & 0.9300 \\
$\mathrm{C}(8)-\mathrm{C}(9)$ & $1.372(11)$ \\
$\mathrm{C}(8)-\mathrm{H}(8)$ & 0.9300 \\
$\mathrm{C}(9)-\mathrm{C}(10)$ & $1.363(11)$ \\
& $\mathrm{S} 13$ \\
\hline
\end{tabular}




\begin{tabular}{|c|c|}
\hline $\mathrm{C}(9)-\mathrm{H}(9)$ & 0.9300 \\
\hline $\mathrm{C}(10)-\mathrm{H}(10)$ & 0.9300 \\
\hline $\mathrm{C}(11)-\mathrm{C}(12)$ & $1.365(9)$ \\
\hline$C(11)-C(16)$ & $1.387(10)$ \\
\hline$C(12)-C(13)$ & $1.366(10)$ \\
\hline $\mathrm{C}(12)-\mathrm{H}(12)$ & 0.9300 \\
\hline$C(13)-C(14)$ & $1.393(12)$ \\
\hline $\mathrm{C}(13)-\mathrm{Cl}(1)$ & $1.744(9)$ \\
\hline$C(14)-C(15)$ & $1.357(11)$ \\
\hline $\mathrm{C}(14)-\mathrm{H}(14)$ & 0.9300 \\
\hline$C(15)-C(16)$ & $1.386(11)$ \\
\hline $\mathrm{C}(15)-\mathrm{H}(15)$ & 0.9300 \\
\hline $\mathrm{C}(16)-\mathrm{H}(16)$ & 0.9300 \\
\hline $\mathrm{C}(17)-\mathrm{O}(1)$ & $1.220(8)$ \\
\hline $\mathrm{C}(17)-\mathrm{N}(2)$ & $1.374(10)$ \\
\hline $\mathrm{C}(17)-\mathrm{N}(1)$ & $1.379(9)$ \\
\hline $\mathrm{C}(18)-\mathrm{N}(2)$ & $1.437(10)$ \\
\hline $\mathrm{C}(18)-\mathrm{H}(18 \mathrm{~A})$ & 0.9600 \\
\hline $\mathrm{C}(18)-\mathrm{H}(18 \mathrm{~B})$ & 0.9600 \\
\hline $\mathrm{C}(18)-\mathrm{H}(18 \mathrm{C})$ & 0.9600 \\
\hline $\mathrm{C}(19)-\mathrm{N}(2)$ & $1.461(10)$ \\
\hline $\mathrm{C}(19)-\mathrm{H}(19 \mathrm{~A})$ & 0.9600 \\
\hline $\mathrm{C}(19)-\mathrm{H}(19 \mathrm{~B})$ & 0.9600 \\
\hline C(19)-H(19C) & 0.9600 \\
\hline$C(20)-C(25)$ & $1.356(9)$ \\
\hline $\mathrm{C}(20)-\mathrm{N}(3)$ & $1.415(9)$ \\
\hline$C(20)-C(21)$ & $1.430(9)$ \\
\hline$C(21)-C(22)$ & $1.371(10)$ \\
\hline $\mathrm{C}(21)-\mathrm{H}(21)$ & 0.9300 \\
\hline$C(22)-C(23)$ & $1.415(10)$ \\
\hline
\end{tabular}




\begin{tabular}{|c|c|}
\hline$C(22)-C(30)$ & $1.496(10)$ \\
\hline$C(23)-C(26)$ & $1.407(10)$ \\
\hline$C(23)-C(24)$ & $1.412(9)$ \\
\hline$C(24)-C(25)$ & $1.415(9)$ \\
\hline$C(24)-C(29)$ & $1.428(10)$ \\
\hline $\mathrm{C}(25)-\mathrm{H}(25)$ & 0.9300 \\
\hline$C(26)-C(27)$ & $1.342(9)$ \\
\hline $\mathrm{C}(26)-\mathrm{H}(26)$ & 0.9300 \\
\hline $\mathrm{C}(27)-\mathrm{C}(28)$ & $1.396(10)$ \\
\hline $\mathrm{C}(27)-\mathrm{H}(27)$ & 0.9300 \\
\hline $\mathrm{C}(28)-\mathrm{C}(29)$ & $1.382(10)$ \\
\hline $\mathrm{C}(28)-\mathrm{H}(28)$ & 0.9300 \\
\hline $\mathrm{C}(29)-\mathrm{H}(29)$ & 0.9300 \\
\hline $\mathrm{C}(30)-\mathrm{C}(31)$ & $1.374(10)$ \\
\hline$C(30)-C(35)$ & $1.405(10)$ \\
\hline $\mathrm{C}(31)-\mathrm{C}(32)$ & $1.378(10)$ \\
\hline $\mathrm{C}(31)-\mathrm{H}(31)$ & 0.9300 \\
\hline $\mathrm{C}(32)-\mathrm{C}(33)$ & $1.382(11)$ \\
\hline $\mathrm{C}(32)-\mathrm{Cl}(2)$ & $1.750(8)$ \\
\hline $\mathrm{C}(33)-\mathrm{C}(34)$ & $1.358(11)$ \\
\hline $\mathrm{C}(33)-\mathrm{H}(33)$ & 0.9300 \\
\hline$C(34)-C(35)$ & $1.387(10)$ \\
\hline $\mathrm{C}(34)-\mathrm{H}(34)$ & 0.9300 \\
\hline $\mathrm{C}(35)-\mathrm{H}(35)$ & 0.9300 \\
\hline $\mathrm{C}(36)-\mathrm{O}(2)$ & $1.218(8)$ \\
\hline $\mathrm{C}(36)-\mathrm{N}(3)$ & $1.368(9)$ \\
\hline $\mathrm{C}(36)-\mathrm{N}(4)$ & $1.379(9)$ \\
\hline $\mathrm{C}(37)-\mathrm{N}(4)$ & $1.444(9)$ \\
\hline $\mathrm{C}(37)-\mathrm{H}(37 \mathrm{~A})$ & 0.9600 \\
\hline $\mathrm{C}(37)-\mathrm{H}(37 \mathrm{~B})$ & 0.9600 \\
\hline
\end{tabular}




\begin{tabular}{|c|c|}
\hline $\mathrm{C}(37)-\mathrm{H}(37 \mathrm{C})$ & 0.9600 \\
\hline $\mathrm{C}(38)-\mathrm{N}(4)$ & $1.451(9)$ \\
\hline $\mathrm{C}(38)-\mathrm{H}(38 \mathrm{~A})$ & 0.9600 \\
\hline $\mathrm{C}(38)-\mathrm{H}(38 \mathrm{~B})$ & 0.9600 \\
\hline $\mathrm{C}(38)-\mathrm{H}(38 \mathrm{C})$ & 0.9600 \\
\hline $\mathrm{N}(1)-\mathrm{H}(1)$ & 0.8600 \\
\hline $\mathrm{N}(3)-\mathrm{H}(3)$ & 0.8600 \\
\hline $\mathrm{C}(6)-\mathrm{C}(1)-\mathrm{C}(2)$ & 119.2(7) \\
\hline $\mathrm{C}(6)-\mathrm{C}(1)-\mathrm{N}(1)$ & $118.5(6)$ \\
\hline $\mathrm{C}(2)-\mathrm{C}(1)-\mathrm{N}(1)$ & $122.3(6)$ \\
\hline $\mathrm{C}(3)-\mathrm{C}(2)-\mathrm{C}(1)$ & $121.5(6)$ \\
\hline $\mathrm{C}(3)-\mathrm{C}(2)-\mathrm{H}(2)$ & 119.3 \\
\hline $\mathrm{C}(1)-\mathrm{C}(2)-\mathrm{H}(2)$ & 119.3 \\
\hline $\mathrm{C}(2)-\mathrm{C}(3)-\mathrm{C}(4)$ & $119.9(6)$ \\
\hline$C(2)-C(3)-C(11)$ & 119.2(6) \\
\hline $\mathrm{C}(4)-\mathrm{C}(3)-\mathrm{C}(11)$ & $121.0(6)$ \\
\hline $\mathrm{C}(7)-\mathrm{C}(4)-\mathrm{C}(5)$ & $118.6(7)$ \\
\hline $\mathrm{C}(7)-\mathrm{C}(4)-\mathrm{C}(3)$ & $124.0(7)$ \\
\hline $\mathrm{C}(5)-\mathrm{C}(4)-\mathrm{C}(3)$ & $117.4(6)$ \\
\hline$C(6)-C(5)-C(4)$ & $120.9(6)$ \\
\hline$C(6)-C(5)-C(10)$ & $120.7(7)$ \\
\hline$C(4)-C(5)-C(10)$ & $118.4(7)$ \\
\hline $\mathrm{C}(1)-\mathrm{C}(6)-\mathrm{C}(5)$ & $121.2(7)$ \\
\hline $\mathrm{C}(1)-\mathrm{C}(6)-\mathrm{H}(6)$ & 119.4 \\
\hline $\mathrm{C}(5)-\mathrm{C}(6)-\mathrm{H}(6)$ & 119.4 \\
\hline$C(8)-C(7)-C(4)$ & $120.7(7)$ \\
\hline $\mathrm{C}(8)-\mathrm{C}(7)-\mathrm{H}(7)$ & 119.6 \\
\hline $\mathrm{C}(4)-\mathrm{C}(7)-\mathrm{H}(7)$ & 119.6 \\
\hline $\mathrm{C}(7)-\mathrm{C}(8)-\mathrm{C}(9)$ & $120.9(8)$ \\
\hline $\mathrm{C}(7)-\mathrm{C}(8)-\mathrm{H}(8)$ & 119.5 \\
\hline
\end{tabular}




\begin{tabular}{|c|c|}
\hline $\mathrm{C}(9)-\mathrm{C}(8)-\mathrm{H}(8)$ & 119.5 \\
\hline $\mathrm{C}(10)-\mathrm{C}(9)-\mathrm{C}(8)$ & $120.9(8)$ \\
\hline $\mathrm{C}(10)-\mathrm{C}(9)-\mathrm{H}(9)$ & 119.6 \\
\hline $\mathrm{C}(8)-\mathrm{C}(9)-\mathrm{H}(9)$ & 119.6 \\
\hline $\mathrm{C}(9)-\mathrm{C}(10)-\mathrm{C}(5)$ & $120.4(7)$ \\
\hline $\mathrm{C}(9)-\mathrm{C}(10)-\mathrm{H}(10)$ & 119.8 \\
\hline $\mathrm{C}(5)-\mathrm{C}(10)-\mathrm{H}(10)$ & 119.8 \\
\hline$C(12)-C(11)-C(16)$ & $118.8(7)$ \\
\hline $\mathrm{C}(12)-\mathrm{C}(11)-\mathrm{C}(3)$ & $121.7(7)$ \\
\hline$C(16)-C(11)-C(3)$ & 119.3(7) \\
\hline$C(11)-C(12)-C(13)$ & $120.5(8)$ \\
\hline $\mathrm{C}(11)-\mathrm{C}(12)-\mathrm{H}(12)$ & 119.7 \\
\hline $\mathrm{C}(13)-\mathrm{C}(12)-\mathrm{H}(12)$ & 119.7 \\
\hline $\mathrm{C}(12)-\mathrm{C}(13)-\mathrm{C}(14)$ & $120.6(8)$ \\
\hline $\mathrm{C}(12)-\mathrm{C}(13)-\mathrm{Cl}(1)$ & $120.3(7)$ \\
\hline $\mathrm{C}(14)-\mathrm{C}(13)-\mathrm{Cl}(1)$ & 119.1(7) \\
\hline$C(15)-C(14)-C(13)$ & $119.5(8)$ \\
\hline $\mathrm{C}(15)-\mathrm{C}(14)-\mathrm{H}(14)$ & 120.2 \\
\hline $\mathrm{C}(13)-\mathrm{C}(14)-\mathrm{H}(14)$ & 120.2 \\
\hline$C(14)-C(15)-C(16)$ & $119.6(8)$ \\
\hline $\mathrm{C}(14)-\mathrm{C}(15)-\mathrm{H}(15)$ & 120.2 \\
\hline $\mathrm{C}(16)-\mathrm{C}(15)-\mathrm{H}(15)$ & 120.2 \\
\hline$C(15)-C(16)-C(11)$ & $120.9(8)$ \\
\hline $\mathrm{C}(15)-\mathrm{C}(16)-\mathrm{H}(16)$ & 119.5 \\
\hline $\mathrm{C}(11)-\mathrm{C}(16)-\mathrm{H}(16)$ & 119.5 \\
\hline $\mathrm{O}(1)-\mathrm{C}(17)-\mathrm{N}(2)$ & $121.8(7)$ \\
\hline $\mathrm{O}(1)-\mathrm{C}(17)-\mathrm{N}(1)$ & $123.9(7)$ \\
\hline $\mathrm{N}(2)-\mathrm{C}(17)-\mathrm{N}(1)$ & $114.3(7)$ \\
\hline $\mathrm{N}(2)-\mathrm{C}(18)-\mathrm{H}(18 \mathrm{~A})$ & 109.5 \\
\hline $\mathrm{N}(2)-\mathrm{C}(18)-\mathrm{H}(18 \mathrm{~B})$ & 109.5 \\
\hline
\end{tabular}




\begin{tabular}{|c|c|}
\hline $\mathrm{H}(18 \mathrm{~A})-\mathrm{C}(18)-\mathrm{H}(18 \mathrm{~B})$ & 109.5 \\
\hline $\mathrm{N}(2)-\mathrm{C}(18)-\mathrm{H}(18 \mathrm{C})$ & 109.5 \\
\hline $\mathrm{H}(18 \mathrm{~A})-\mathrm{C}(18)-\mathrm{H}(18 \mathrm{C})$ & 109.5 \\
\hline $\mathrm{H}(18 \mathrm{~B})-\mathrm{C}(18)-\mathrm{H}(18 \mathrm{C})$ & 109.5 \\
\hline $\mathrm{N}(2)-\mathrm{C}(19)-\mathrm{H}(19 \mathrm{~A})$ & 109.5 \\
\hline $\mathrm{N}(2)-\mathrm{C}(19)-\mathrm{H}(19 \mathrm{~B})$ & 109.5 \\
\hline $\mathrm{H}(19 \mathrm{~A})-\mathrm{C}(19)-\mathrm{H}(19 \mathrm{~B})$ & 109.5 \\
\hline $\mathrm{N}(2)-\mathrm{C}(19)-\mathrm{H}(19 \mathrm{C})$ & 109.5 \\
\hline $\mathrm{H}(19 \mathrm{~A})-\mathrm{C}(19)-\mathrm{H}(19 \mathrm{C})$ & 109.5 \\
\hline $\mathrm{H}(19 \mathrm{~B})-\mathrm{C}(19)-\mathrm{H}(19 \mathrm{C})$ & 109.5 \\
\hline $\mathrm{C}(25)-\mathrm{C}(20)-\mathrm{N}(3)$ & $126.7(7)$ \\
\hline$C(25)-C(20)-C(21)$ & $119.0(7)$ \\
\hline $\mathrm{N}(3)-\mathrm{C}(20)-\mathrm{C}(21)$ & $114.3(7)$ \\
\hline$C(22)-C(21)-C(20)$ & $121.4(7)$ \\
\hline $\mathrm{C}(22)-\mathrm{C}(21)-\mathrm{H}(21)$ & 119.3 \\
\hline $\mathrm{C}(20)-\mathrm{C}(21)-\mathrm{H}(21)$ & 119.3 \\
\hline $\mathrm{C}(21)-\mathrm{C}(22)-\mathrm{C}(23)$ & $120.4(7)$ \\
\hline $\mathrm{C}(21)-\mathrm{C}(22)-\mathrm{C}(30)$ & $115.3(7)$ \\
\hline$C(23)-C(22)-C(30)$ & $124.2(7)$ \\
\hline$C(26)-C(23)-C(24)$ & $118.5(7)$ \\
\hline$C(26)-C(23)-C(22)$ & $123.9(7)$ \\
\hline$C(24)-C(23)-C(22)$ & $117.5(7)$ \\
\hline$C(23)-C(24)-C(25)$ & $121.3(7)$ \\
\hline$C(23)-C(24)-C(29)$ & $118.8(7)$ \\
\hline$C(25)-C(24)-C(29)$ & $119.8(7)$ \\
\hline$C(20)-C(25)-C(24)$ & $120.2(7)$ \\
\hline $\mathrm{C}(20)-\mathrm{C}(25)-\mathrm{H}(25)$ & 119.9 \\
\hline $\mathrm{C}(24)-\mathrm{C}(25)-\mathrm{H}(25)$ & 119.9 \\
\hline$C(27)-C(26)-C(23)$ & $121.4(7)$ \\
\hline $\mathrm{C}(27)-\mathrm{C}(26)-\mathrm{H}(26)$ & 119.3 \\
\hline
\end{tabular}




\begin{tabular}{|c|c|}
\hline $\mathrm{C}(23)-\mathrm{C}(26)-\mathrm{H}(26)$ & 119.3 \\
\hline $\mathrm{C}(26)-\mathrm{C}(27)-\mathrm{C}(28)$ & $121.9(8)$ \\
\hline $\mathrm{C}(26)-\mathrm{C}(27)-\mathrm{H}(27)$ & 119.0 \\
\hline $\mathrm{C}(28)-\mathrm{C}(27)-\mathrm{H}(27)$ & 119.0 \\
\hline $\mathrm{C}(29)-\mathrm{C}(28)-\mathrm{C}(27)$ & $118.7(8)$ \\
\hline $\mathrm{C}(29)-\mathrm{C}(28)-\mathrm{H}(28)$ & 120.6 \\
\hline $\mathrm{C}(27)-\mathrm{C}(28)-\mathrm{H}(28)$ & 120.6 \\
\hline $\mathrm{C}(28)-\mathrm{C}(29)-\mathrm{C}(24)$ & $120.6(7)$ \\
\hline $\mathrm{C}(28)-\mathrm{C}(29)-\mathrm{H}(29)$ & 119.7 \\
\hline $\mathrm{C}(24)-\mathrm{C}(29)-\mathrm{H}(29)$ & 119.7 \\
\hline $\mathrm{C}(31)-\mathrm{C}(30)-\mathrm{C}(35)$ & $118.2(7)$ \\
\hline $\mathrm{C}(31)-\mathrm{C}(30)-\mathrm{C}(22)$ & $119.7(7)$ \\
\hline $\mathrm{C}(35)-\mathrm{C}(30)-\mathrm{C}(22)$ & $122.1(7)$ \\
\hline $\mathrm{C}(30)-\mathrm{C}(31)-\mathrm{C}(32)$ & $120.7(8)$ \\
\hline $\mathrm{C}(30)-\mathrm{C}(31)-\mathrm{H}(31)$ & 119.6 \\
\hline $\mathrm{C}(32)-\mathrm{C}(31)-\mathrm{H}(31)$ & 119.6 \\
\hline $\mathrm{C}(31)-\mathrm{C}(32)-\mathrm{C}(33)$ & $121.5(8)$ \\
\hline $\mathrm{C}(31)-\mathrm{C}(32)-\mathrm{Cl}(2)$ & $118.9(7)$ \\
\hline $\mathrm{C}(33)-\mathrm{C}(32)-\mathrm{Cl}(2)$ & 119.7(7) \\
\hline$C(34)-C(33)-C(32)$ & $118.1(8)$ \\
\hline $\mathrm{C}(34)-\mathrm{C}(33)-\mathrm{H}(33)$ & 120.9 \\
\hline $\mathrm{C}(32)-\mathrm{C}(33)-\mathrm{H}(33)$ & 120.9 \\
\hline $\mathrm{C}(33)-\mathrm{C}(34)-\mathrm{C}(35)$ & $121.8(8)$ \\
\hline $\mathrm{C}(33)-\mathrm{C}(34)-\mathrm{H}(34)$ & 119.1 \\
\hline $\mathrm{C}(35)-\mathrm{C}(34)-\mathrm{H}(34)$ & 119.1 \\
\hline $\mathrm{C}(34)-\mathrm{C}(35)-\mathrm{C}(30)$ & 119.7(8) \\
\hline $\mathrm{C}(34)-\mathrm{C}(35)-\mathrm{H}(35)$ & 120.1 \\
\hline $\mathrm{C}(30)-\mathrm{C}(35)-\mathrm{H}(35)$ & 120.1 \\
\hline $\mathrm{O}(2)-\mathrm{C}(36)-\mathrm{N}(3)$ & $123.8(7)$ \\
\hline $\mathrm{O}(2)-\mathrm{C}(36)-\mathrm{N}(4)$ & $121.7(7)$ \\
\hline
\end{tabular}




\begin{tabular}{|c|c|}
\hline $\mathrm{N}(3)-\mathrm{C}(36)-\mathrm{N}(4)$ & $114.5(7)$ \\
\hline $\mathrm{N}(4)-\mathrm{C}(37)-\mathrm{H}(37 \mathrm{~A})$ & 109.5 \\
\hline $\mathrm{N}(4)-\mathrm{C}(37)-\mathrm{H}(37 \mathrm{~B})$ & 109.5 \\
\hline $\mathrm{H}(37 \mathrm{~A})-\mathrm{C}(37)-\mathrm{H}(37 \mathrm{~B})$ & 109.5 \\
\hline $\mathrm{N}(4)-\mathrm{C}(37)-\mathrm{H}(37 \mathrm{C})$ & 109.5 \\
\hline $\mathrm{H}(37 \mathrm{~A})-\mathrm{C}(37)-\mathrm{H}(37 \mathrm{C})$ & 109.5 \\
\hline $\mathrm{H}(37 \mathrm{~B})-\mathrm{C}(37)-\mathrm{H}(37 \mathrm{C})$ & 109.5 \\
\hline $\mathrm{N}(4)-\mathrm{C}(38)-\mathrm{H}(38 \mathrm{~A})$ & 109.5 \\
\hline $\mathrm{N}(4)-\mathrm{C}(38)-\mathrm{H}(38 \mathrm{~B})$ & 109.5 \\
\hline $\mathrm{H}(38 \mathrm{~A})-\mathrm{C}(38)-\mathrm{H}(38 \mathrm{~B})$ & 109.5 \\
\hline $\mathrm{N}(4)-\mathrm{C}(38)-\mathrm{H}(38 \mathrm{C})$ & 109.5 \\
\hline $\mathrm{H}(38 \mathrm{~A})-\mathrm{C}(38)-\mathrm{H}(38 \mathrm{C})$ & 109.5 \\
\hline $\mathrm{H}(38 \mathrm{~B})-\mathrm{C}(38)-\mathrm{H}(38 \mathrm{C})$ & 109.5 \\
\hline $\mathrm{C}(17)-\mathrm{N}(1)-\mathrm{C}(1)$ & $125.5(6)$ \\
\hline $\mathrm{C}(17)-\mathrm{N}(1)-\mathrm{H}(1)$ & 117.3 \\
\hline $\mathrm{C}(1)-\mathrm{N}(1)-\mathrm{H}(1)$ & 117.3 \\
\hline $\mathrm{C}(17)-\mathrm{N}(2)-\mathrm{C}(18)$ & $125.4(7)$ \\
\hline $\mathrm{C}(17)-\mathrm{N}(2)-\mathrm{C}(19)$ & $118.5(7)$ \\
\hline $\mathrm{C}(18)-\mathrm{N}(2)-\mathrm{C}(19)$ & $116.1(8)$ \\
\hline $\mathrm{C}(36)-\mathrm{N}(3)-\mathrm{C}(20)$ & $126.5(6)$ \\
\hline $\mathrm{C}(36)-\mathrm{N}(3)-\mathrm{H}(3)$ & 116.7 \\
\hline $\mathrm{C}(20)-\mathrm{N}(3)-\mathrm{H}(3)$ & 116.7 \\
\hline $\mathrm{C}(36)-\mathrm{N}(4)-\mathrm{C}(37)$ & $124.8(6)$ \\
\hline $\mathrm{C}(36)-\mathrm{N}(4)-\mathrm{C}(38)$ & $119.2(7)$ \\
\hline $\mathrm{C}(37)-\mathrm{N}(4)-\mathrm{C}(38)$ & $116.0(6)$ \\
\hline
\end{tabular}

Symmetry transformations used to generate equivalent atoms:

Table 4. Anisotropic displacement parameters $\left(\mathrm{A}^{\wedge} 2 \times 10^{\wedge} 3\right)$ for $3 \mathrm{ah}$. The anisotropic displacement factor exponent takes the form: $-2 \mathrm{pi}^{\wedge} 2\left[\mathrm{~h}^{\wedge} 2 \mathrm{a}^{* \wedge} 2 \mathrm{U} 11+\ldots+2 \mathrm{~h} \mathrm{k} \mathrm{a}^{*} \mathrm{~b}^{*} \mathrm{U} 12\right]$ 


\begin{tabular}{|c|c|c|c|c|c|c|}
\hline & U11 & $\mathrm{U} 22$ & U33 & U23 & U13 & U12 \\
\hline $\mathrm{C}(1)$ & $39(4)$ & $44(5)$ & $37(4)$ & $-1(3)$ & $1(4)$ & $-7(4)$ \\
\hline $\mathrm{C}(2)$ & $45(5)$ & $46(5)$ & $34(4)$ & $5(4)$ & $4(4)$ & $-5(4)$ \\
\hline $\mathrm{C}(3)$ & $39(4)$ & $44(5)$ & $44(4)$ & $1(4)$ & $8(4)$ & $-6(4)$ \\
\hline$C(4)$ & $37(4)$ & $44(5)$ & $37(4)$ & $-3(3)$ & $2(3)$ & $-3(4)$ \\
\hline$C(5)$ & $43(5)$ & $42(5)$ & $38(4)$ & $-2(3)$ & $5(4)$ & $-4(4)$ \\
\hline$C(6)$ & $44(5)$ & $43(5)$ & $42(4)$ & $0(4)$ & $11(4)$ & $-8(4)$ \\
\hline $\mathrm{C}(7)$ & $39(5)$ & $68(6)$ & $49(5)$ & $-1(4)$ & $-2(4)$ & $-10(4)$ \\
\hline $\mathrm{C}(8)$ & $64(6)$ & $76(6)$ & $47(5)$ & $1(5)$ & $-9(5)$ & $-21(5)$ \\
\hline$C(9)$ & $91(8)$ & $84(7)$ & $34(5)$ & $-2(5)$ & $9(5)$ & $-7(6)$ \\
\hline$C(10)$ & $67(6)$ & $64(6)$ & $37(5)$ & $-1(4)$ & $13(4)$ & $-15(4)$ \\
\hline$C(11)$ & $35(4)$ & $52(5)$ & $38(4)$ & $2(4)$ & $4(4)$ & $-8(4)$ \\
\hline$C(12)$ & $40(5)$ & $49(5)$ & $67(5)$ & $5(4)$ & $14(4)$ & $-8(4)$ \\
\hline$C(13)$ & $46(5)$ & $63(6)$ & $71(6)$ & $5(5)$ & $-2(5)$ & $-1(5)$ \\
\hline$C(14)$ & $70(7)$ & $85(8)$ & $71(6)$ & $18(6)$ & $3(6)$ & $-29(6)$ \\
\hline$C(15)$ & $81(7)$ & $86(7)$ & $63(6)$ & $-9(5)$ & $32(5)$ & $-27(6)$ \\
\hline$C(16)$ & $58(6)$ & $67(6)$ & $46(5)$ & $-3(4)$ & $8(4)$ & $-12(5)$ \\
\hline$C(17)$ & $41(5)$ & $58(6)$ & $42(5)$ & $-4(4)$ & $2(4)$ & $9(4)$ \\
\hline $\mathrm{C}(18)$ & 133(8) & $112(7)$ & $93(6)$ & $5(6)$ & $-12(6)$ & $-52(6)$ \\
\hline C(19) & $84(7)$ & 109(8) & $41(5)$ & $1(5)$ & $-22(5)$ & $-1(6)$ \\
\hline$C(20)$ & $38(4)$ & $46(5)$ & $44(4)$ & $4(4)$ & $11(4)$ & $-3(4)$ \\
\hline$C(21)$ & $53(5)$ & $43(5)$ & $52(5)$ & $7(4)$ & $6(4)$ & $-2(4)$ \\
\hline$C(22)$ & $46(5)$ & $49(5)$ & $42(4)$ & $-1(4)$ & $9(4)$ & $-11(4)$ \\
\hline$C(23)$ & $43(5)$ & $46(5)$ & $30(4)$ & $-7(3)$ & $3(4)$ & $-5(4)$ \\
\hline$C(24)$ & $43(5)$ & $41(5)$ & $44(4)$ & $-4(4)$ & $9(4)$ & $-2(4)$ \\
\hline$C(25)$ & $45(5)$ & $44(5)$ & $53(5)$ & $9(4)$ & $6(4)$ & $-5(4)$ \\
\hline$C(26)$ & $47(5)$ & $57(6)$ & $40(4)$ & $-1(4)$ & $6(4)$ & $-9(4)$ \\
\hline$C(27)$ & $40(5)$ & $68(6)$ & $51(5)$ & $-7(4)$ & $5(4)$ & $3(5)$ \\
\hline
\end{tabular}




\begin{tabular}{|c|c|c|c|c|c|c|}
\hline $\mathrm{C}(28)$ & $43(5)$ & $60(6)$ & $53(5)$ & $-5(4)$ & $-2(4)$ & 6(4) \\
\hline $\mathrm{C}(29)$ & 49(5) & $50(5)$ & $40(4)$ & $-3(4)$ & $-2(4)$ & $-7(4)$ \\
\hline $\mathrm{C}(30)$ & $58(5)$ & $50(5)$ & $45(5)$ & $4(4)$ & $17(4)$ & $-8(4)$ \\
\hline $\mathrm{C}(31)$ & $53(5)$ & $58(6)$ & $59(5)$ & $15(4)$ & 2(4) & $-7(4)$ \\
\hline $\mathrm{C}(32)$ & 77(7) & $64(6)$ & $49(5)$ & 19(5) & $4(5)$ & $-11(5)$ \\
\hline $\mathrm{C}(33)$ & $80(7)$ & $67(6)$ & $64(6)$ & $5(5)$ & $12(5)$ & $-24(5)$ \\
\hline $\mathrm{C}(34)$ & $65(6)$ & $75(7)$ & $63(6)$ & $-3(5)$ & 1(5) & $-29(5)$ \\
\hline $\mathrm{C}(35)$ & $58(6)$ & $62(6)$ & $46(5)$ & $-1(4)$ & $-2(4)$ & $-17(5)$ \\
\hline $\mathrm{C}(36)$ & $40(5)$ & $54(6)$ & $44(5)$ & 2(4) & 2(4) & $-5(4)$ \\
\hline C(37) & $65(6)$ & $65(6)$ & 111(8) & $17(6)$ & $34(6)$ & $15(5)$ \\
\hline $\mathrm{C}(38)$ & $41(5)$ & $68(6)$ & $100(7)$ & $2(5)$ & $23(5)$ & $-9(4)$ \\
\hline $\mathrm{N}(1)$ & $49(4)$ & $53(4)$ & $43(4)$ & $3(3)$ & $-2(3)$ & $-18(3)$ \\
\hline $\mathrm{N}(2)$ & $72(5)$ & $85(6)$ & $46(4)$ & $-1(4)$ & $-14(4)$ & $-24(4)$ \\
\hline $\mathrm{N}(3)$ & $40(4)$ & 49(4) & $75(5)$ & $10(3)$ & $14(4)$ & $4(3)$ \\
\hline $\mathrm{N}(4)$ & $44(4)$ & $51(4)$ & $73(5)$ & $10(4)$ & $17(4)$ & $2(3)$ \\
\hline $\mathrm{O}(1)$ & $53(4)$ & $59(4)$ & $49(3)$ & $7(3)$ & $-5(3)$ & $-3(3)$ \\
\hline $\mathrm{O}(2)$ & $44(3)$ & $46(3)$ & $80(4)$ & $4(3)$ & $9(3)$ & $-3(3)$ \\
\hline $\mathrm{Cl}(1)$ & $70(2)$ & $64(2)$ & $138(2)$ & $-8(2)$ & $-2(2)$ & $-13(1)$ \\
\hline $\mathrm{Cl}(2)$ & $114(2)$ & $105(2)$ & $90(2)$ & 49(2) & $-16(2)$ & $-31(2)$ \\
\hline
\end{tabular}

Table 5. Hydrogen coordinates ( $\left.\times 10^{\wedge} 4\right)$ and isotropic displacement parameters $\left(\mathrm{A}^{\wedge} 2 \times 10^{\wedge} 3\right)$ for 3 ah.

\begin{tabular}{lcccc}
\hline & $\mathrm{x}$ & $\mathrm{y}$ & $\mathrm{z}$ & $\mathrm{U}(\mathrm{eq})$ \\
\hline $\mathrm{H}(2)$ & 6520 & 8379 & 8027 & 51 \\
$\mathrm{H}(6)$ & 5262 & 7322 & 9765 & 51 \\
$\mathrm{H}(7)$ & 8636 & 8958 & 10710 & 64 \\
$\mathrm{H}(8)$ & 8561 & 8688 & 12092 & 78 \\
$\mathrm{H}(9)$ & 7276 & 7936 & 12387 & 84 \\
$\mathrm{H}(10)$ & 6034 & 7440 & 11300 & 67 \\
& & & &
\end{tabular}




\begin{tabular}{|c|c|c|c|c|}
\hline $\mathrm{H}(12)$ & 7815 & 9953 & 9730 & 62 \\
\hline $\mathrm{H}(14)$ & 9868 & 10403 & 8265 & 92 \\
\hline $\mathrm{H}(15)$ & 9669 & 9230 & 7708 & 89 \\
\hline $\mathrm{H}(16)$ & 8527 & 8419 & 8155 & 69 \\
\hline $\mathrm{H}(18 \mathrm{~A})$ & 2891 & 6446 & 6635 & 177 \\
\hline $\mathrm{H}(18 \mathrm{~B})$ & 3168 & 6706 & 7598 & 177 \\
\hline $\mathrm{H}(18 \mathrm{C})$ & 3995 & 6268 & 7213 & 177 \\
\hline $\mathrm{H}(19 \mathrm{~A})$ & 3346 & 7160 & 5563 & 125 \\
\hline $\mathrm{H}(19 \mathrm{~B})$ & 3786 & 7955 & 5804 & 125 \\
\hline $\mathrm{H}(19 \mathrm{C})$ & 2673 & 7737 & 5941 & 125 \\
\hline $\mathrm{H}(21)$ & 3361 & 9121 & 6689 & 60 \\
\hline $\mathrm{H}(25)$ & 3586 & 11197 & 5863 & 57 \\
\hline $\mathrm{H}(26)$ & 44 & 10135 & 6434 & 58 \\
\hline $\mathrm{H}(27)$ & -648 & 11264 & 6114 & 64 \\
\hline $\mathrm{H}(28)$ & 328 & 12223 & 5718 & 65 \\
\hline $\mathrm{H}(29)$ & 2054 & 12011 & 5647 & 58 \\
\hline $\mathrm{H}(31)$ & 2489 & 8844 & 7895 & 70 \\
\hline $\mathrm{H}(33)$ & 224 & 7310 & 7436 & 85 \\
\hline H(34) & -492 & 7882 & 6161 & 84 \\
\hline $\mathrm{H}(35)$ & 224 & 8952 & 5750 & 69 \\
\hline $\mathrm{H}(37 \mathrm{~A})$ & 6010 & 9005 & 5872 & 117 \\
\hline $\mathrm{H}(37 \mathrm{~B})$ & 6649 & 9160 & 6809 & 117 \\
\hline $\mathrm{H}(37 \mathrm{C})$ & 7221 & 9143 & 6039 & 117 \\
\hline $\mathrm{H}(38 \mathrm{~A})$ & 7647 & 10224 & 5578 & 103 \\
\hline $\mathrm{H}(38 \mathrm{~B})$ & 7748 & 10588 & 6487 & 103 \\
\hline $\mathrm{H}(38 \mathrm{C})$ & 7033 & 10952 & 5674 & 103 \\
\hline $\mathrm{H}(1)$ & 4757 & 7033 & 8265 & 61 \\
\hline $\mathrm{H}(3)$ & 4890 & 9512 & 6430 & 65 \\
\hline
\end{tabular}




\section{References}

[1] A. Cappelli, M. Anzini, S. Vomero, J. Med. Chem. 1999, 42, 1556-1575.

[2] T. Nishikata, A. Abela, S. Huang, B. Lipshutz, J. Am. Chem. Soc.2010, 132 , 4978-4979.

[3]T. Nishikata, A. Abela, B. Lipshutz, Angew. Chem. Int. Ed. 2010, 49, 781 -784.

[4] D. Xue, J. Li, Y. Liu, W. Han, Z. Zhang, C. Wang, J. Xiao, Synlett 2012, 23, 1941-1946.

[5] M. Manna, A. Hossian, R. Jana, Org. Lett., 2015, 17, 672-675. 
(F) Copies of ${ }^{1} \mathrm{H}$ and ${ }^{13} \mathrm{C}$ spectra

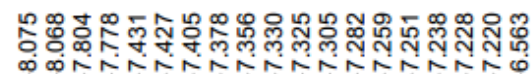

里NNNNNNNNNNNNND

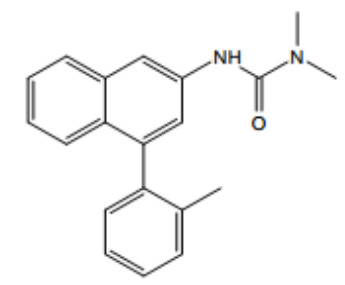

$3 a a$

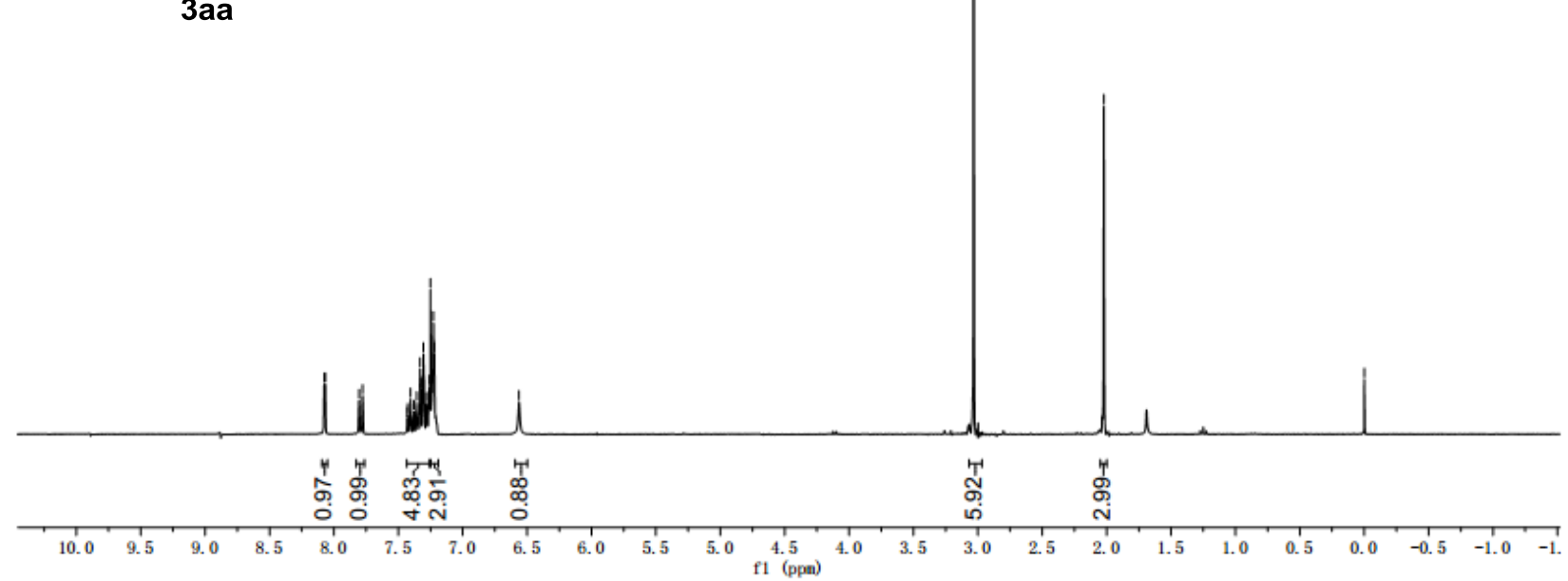

웜ํำ

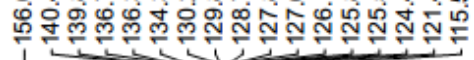

ซฺฺุ ำ

Fin

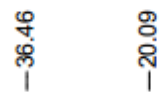

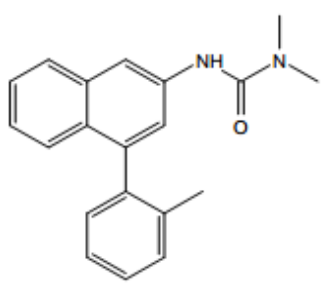

$3 a a$

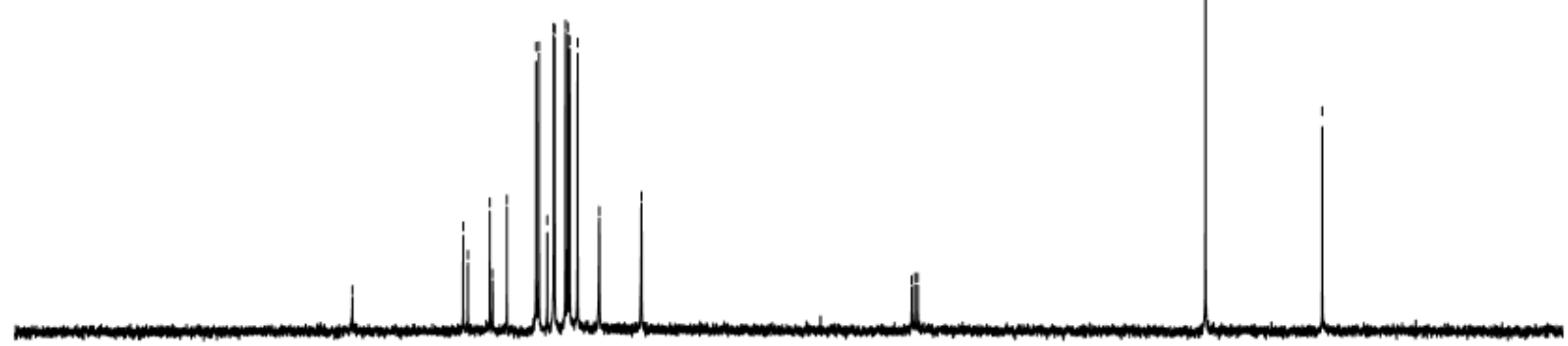




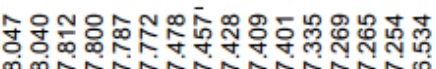

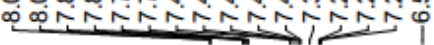<smiles>CCC(=O)NC1CC2CCCCC2C(c2ccccc2)C1</smiles>

$3 a b$
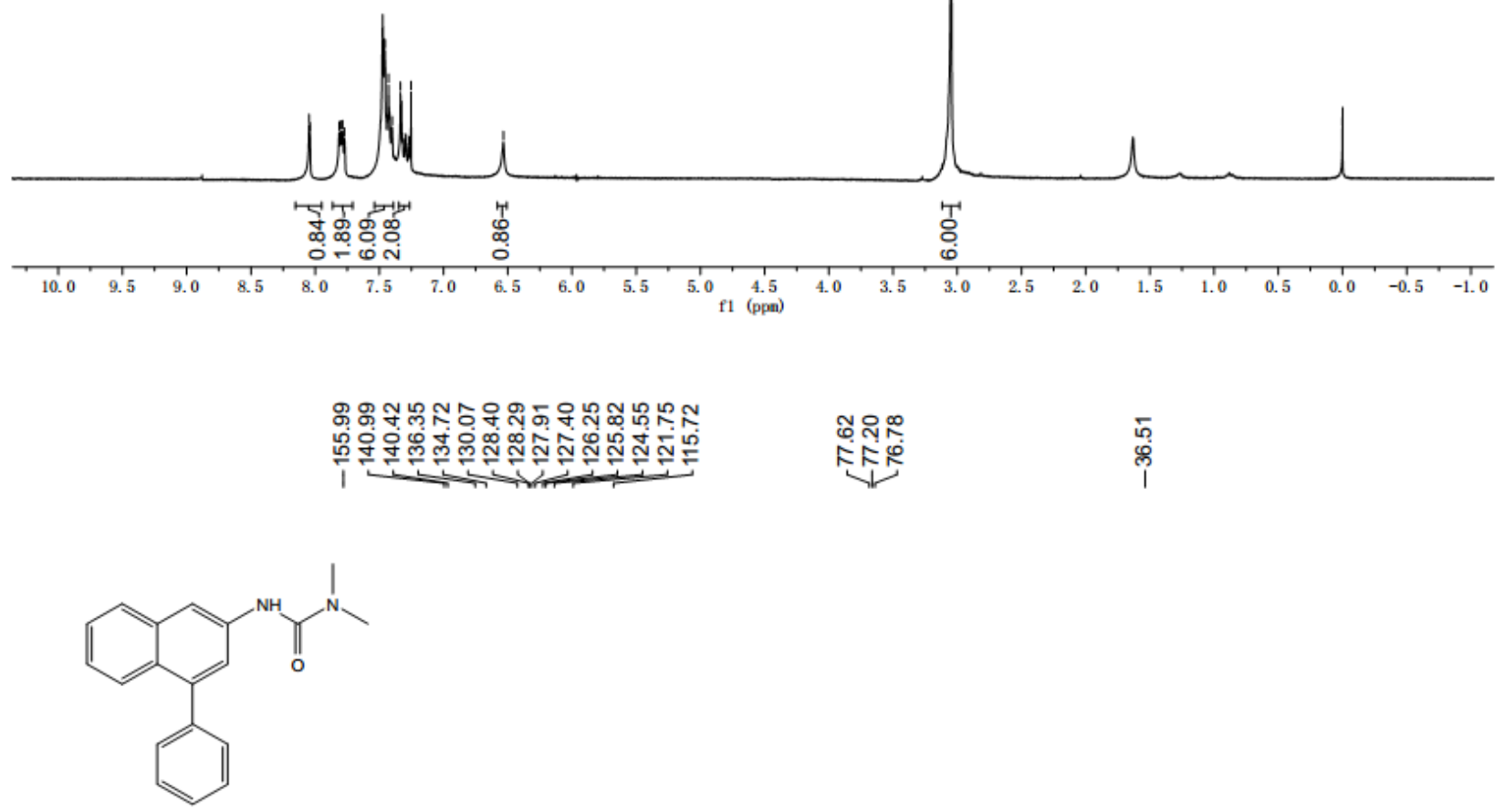

$3 a b$

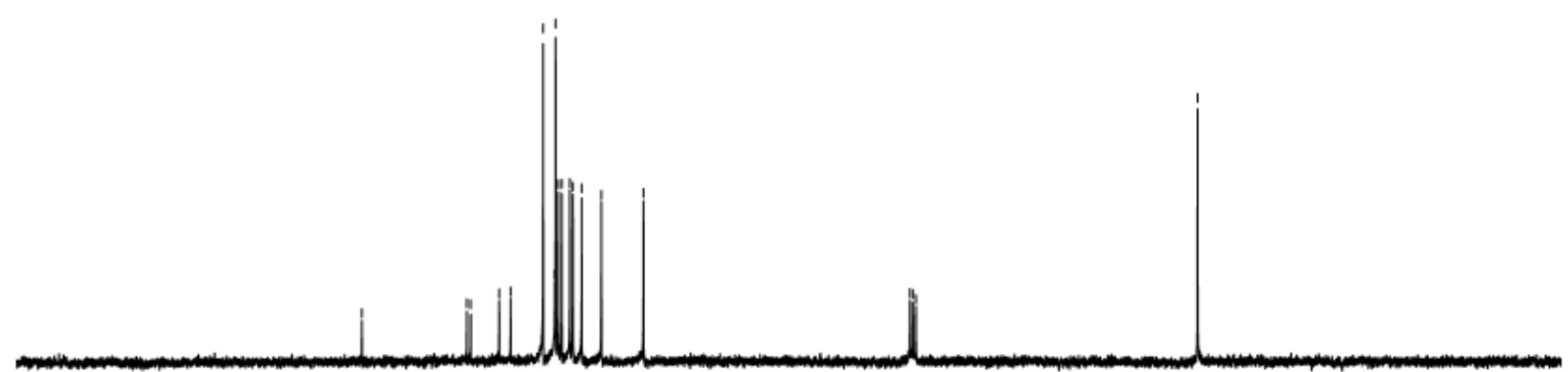




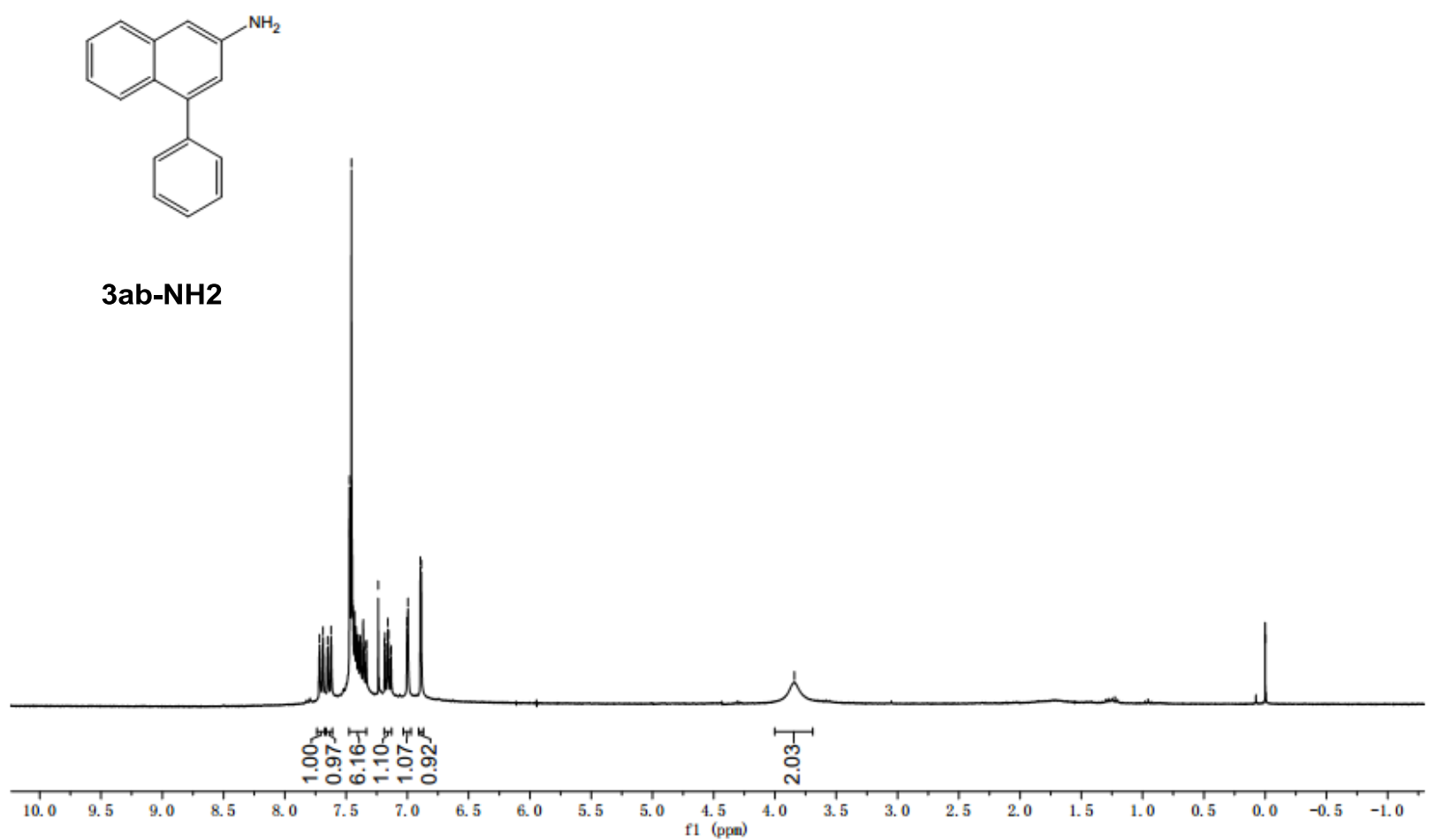

NRू<smiles>Nc1cc(-c2ccccc2)c2ccccc2c1</smiles>

3ab-NH2

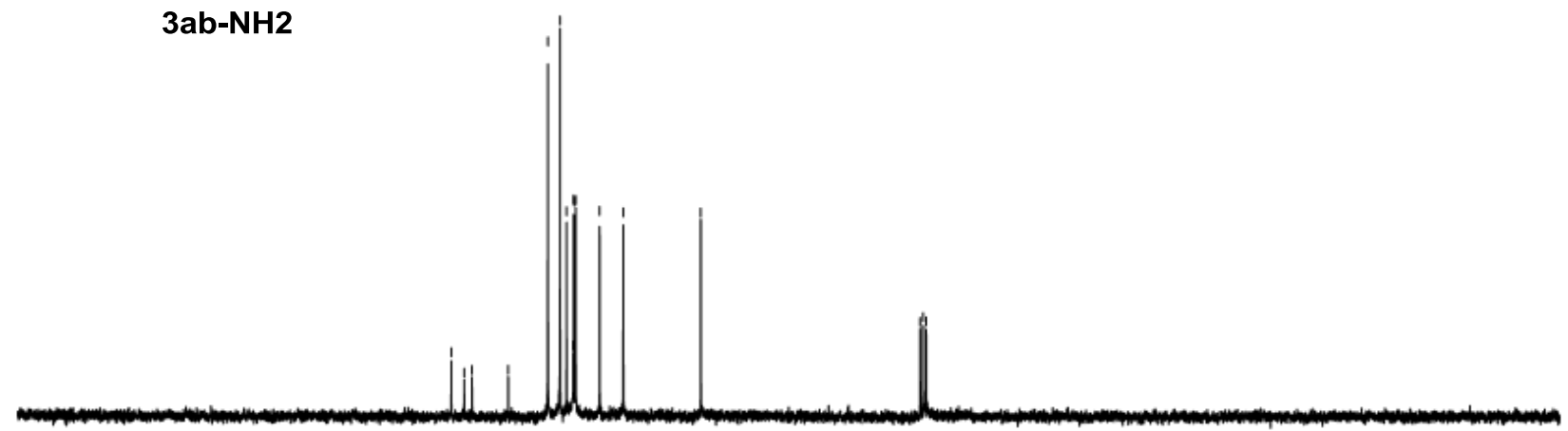




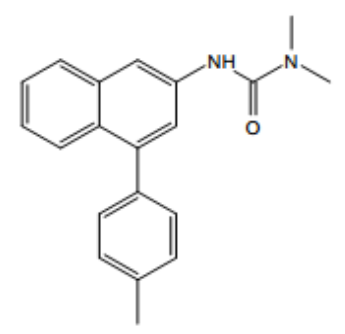

3ac

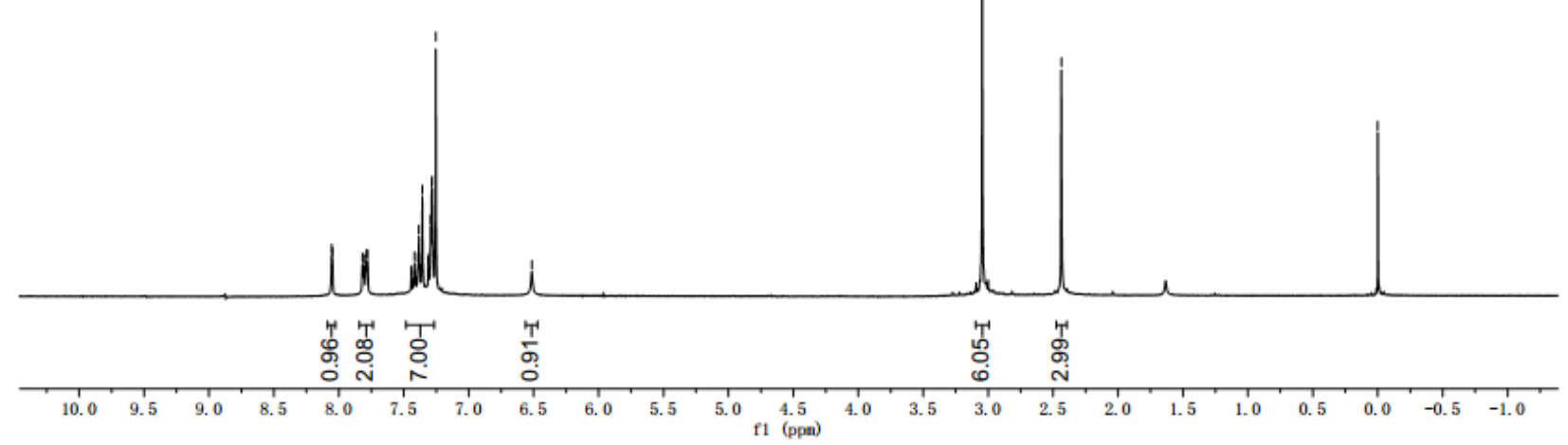<smiles>Cc1ccc(-c2cc(NC(=O)N(C)C)cc3ccccc23)cc1</smiles>

3ac

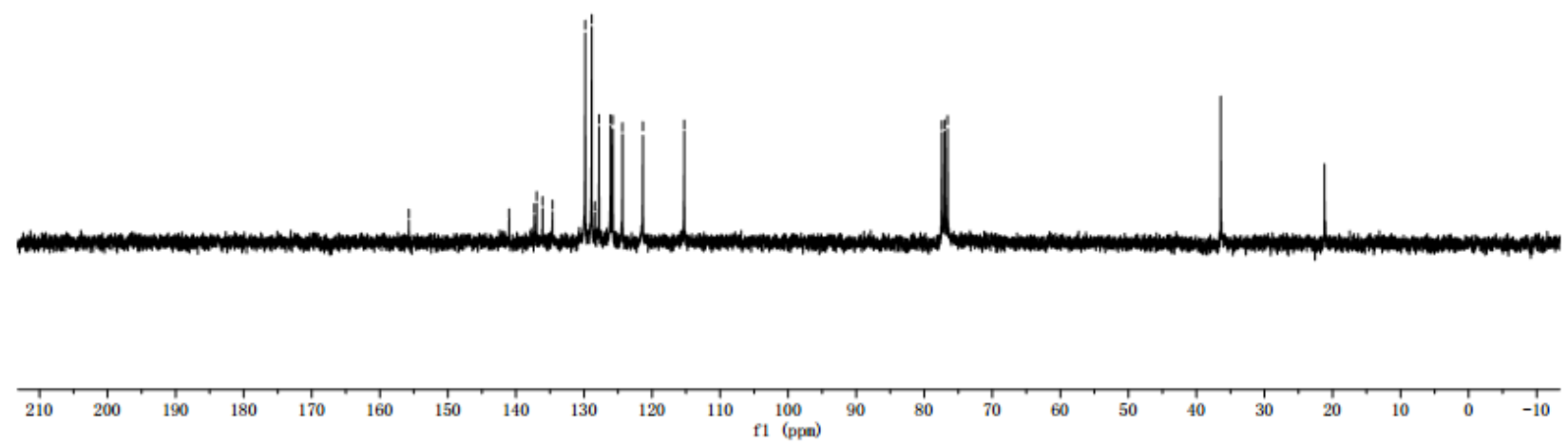



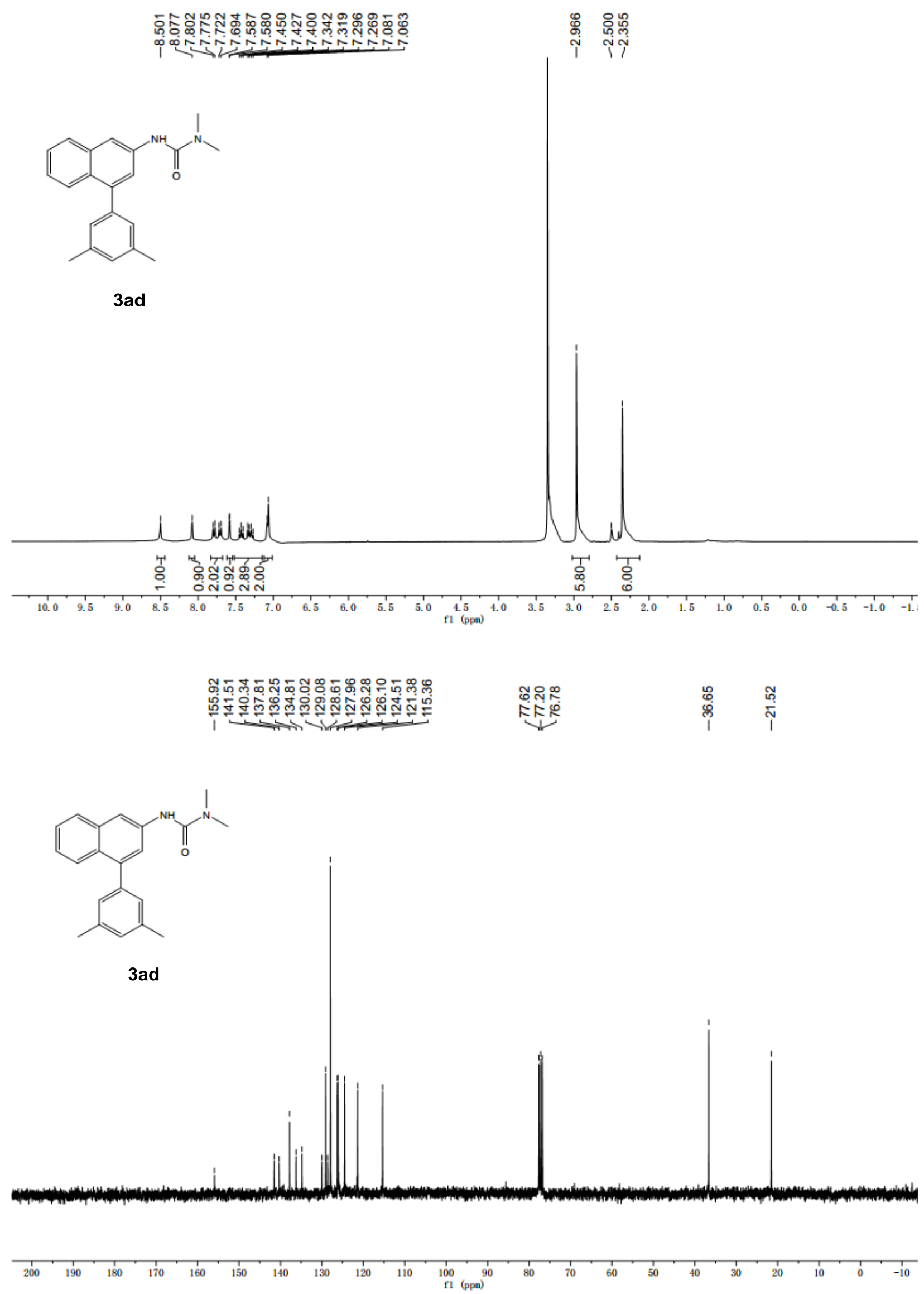
<smiles>COc1ccc(-c2cc(NC(=O)N(C)C)cc3ccccc23)cc1</smiles>

$3 a e$

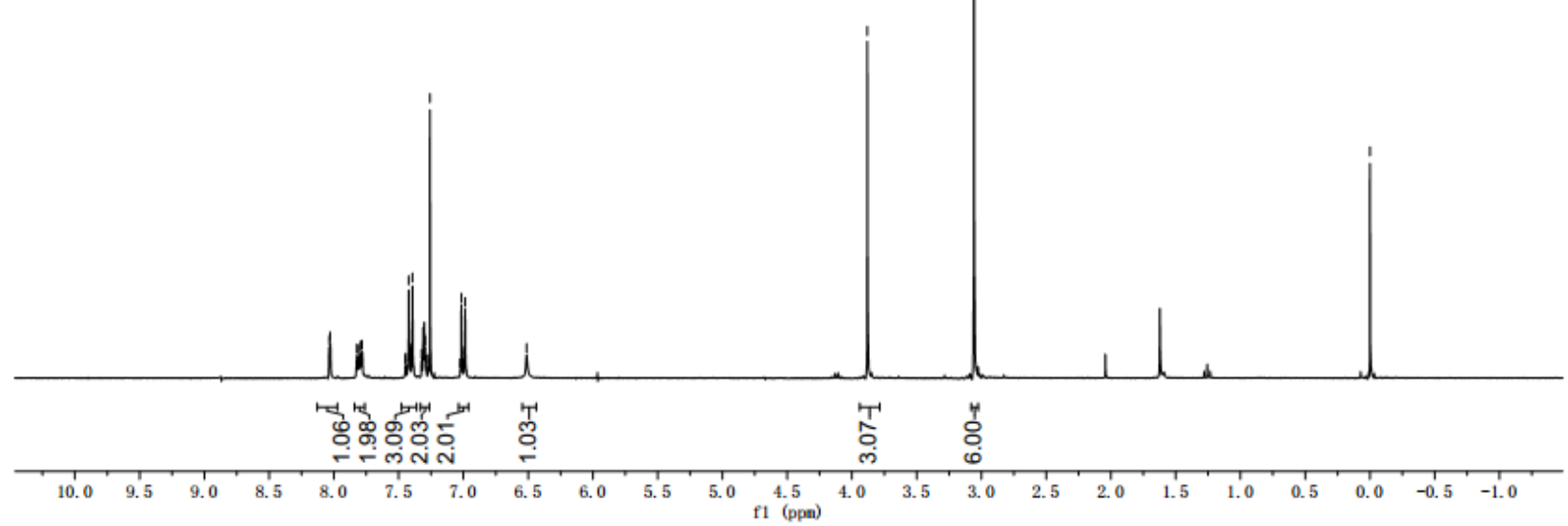

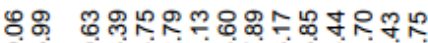

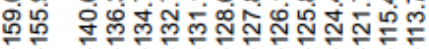

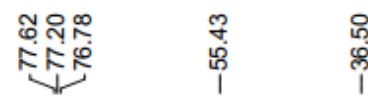

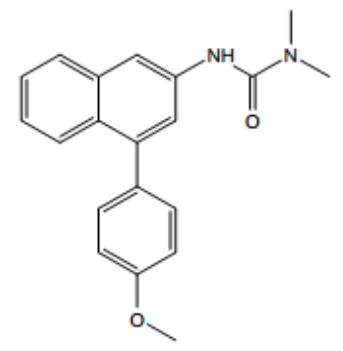

$3 a e$

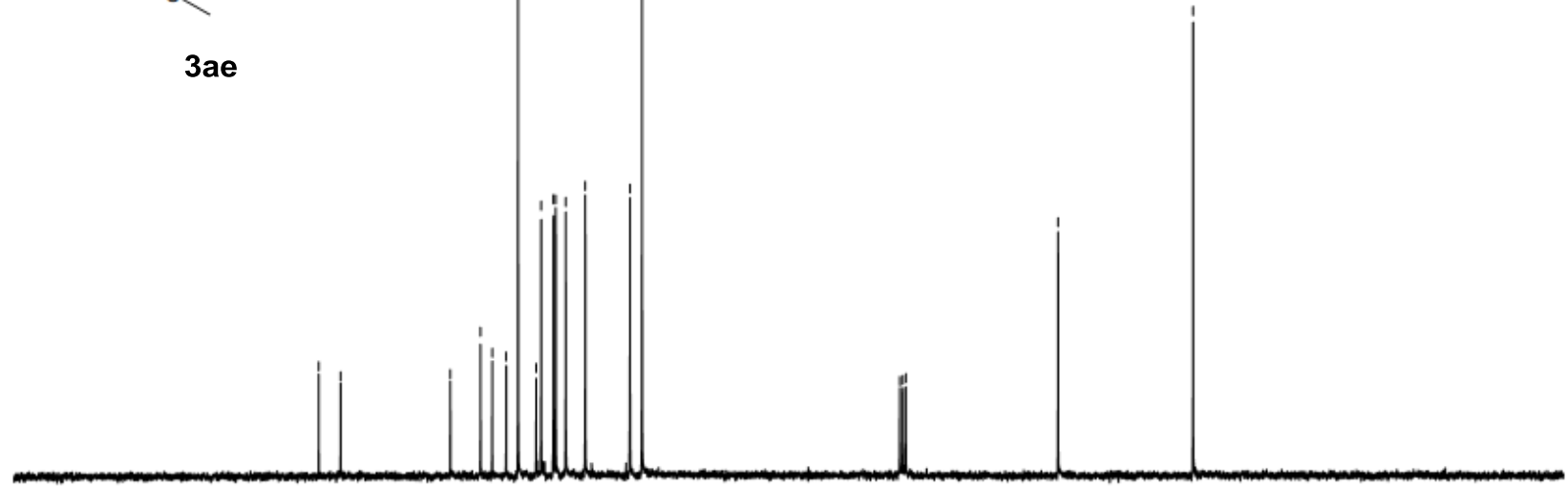

200
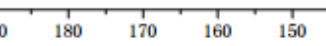

${ }_{130}^{1} \cdot 120$

$100 \quad 90$

8070

60

30

${ }_{20}^{1} 10 \quad 0 \quad-10$ 


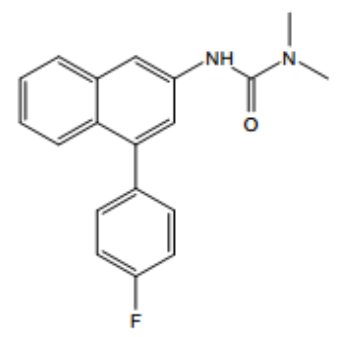

3af

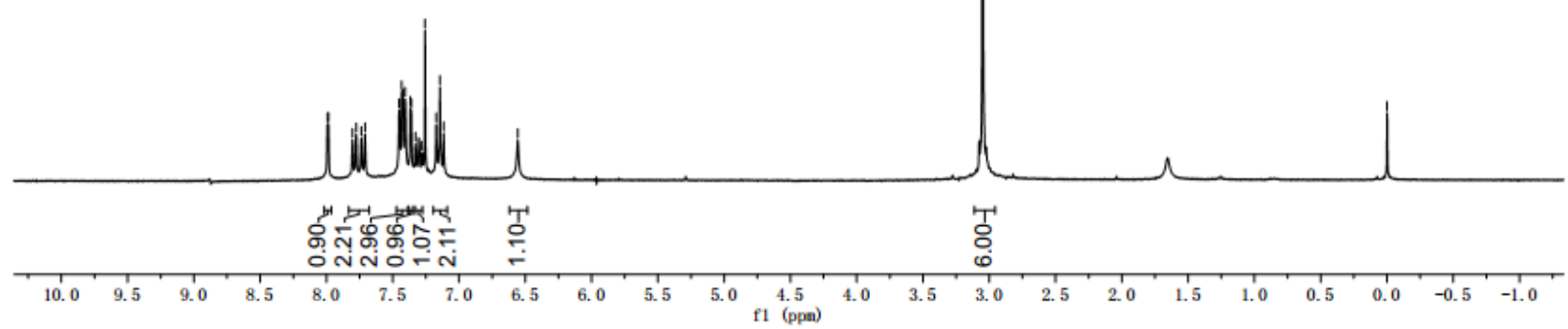

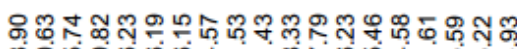

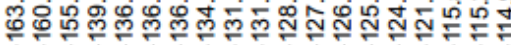

눈단

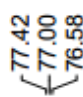

莳
i<smiles>CN(C)C(=O)Nc1cc(-c2ccc(F)cc2)c2ccccc2c1</smiles>

3af

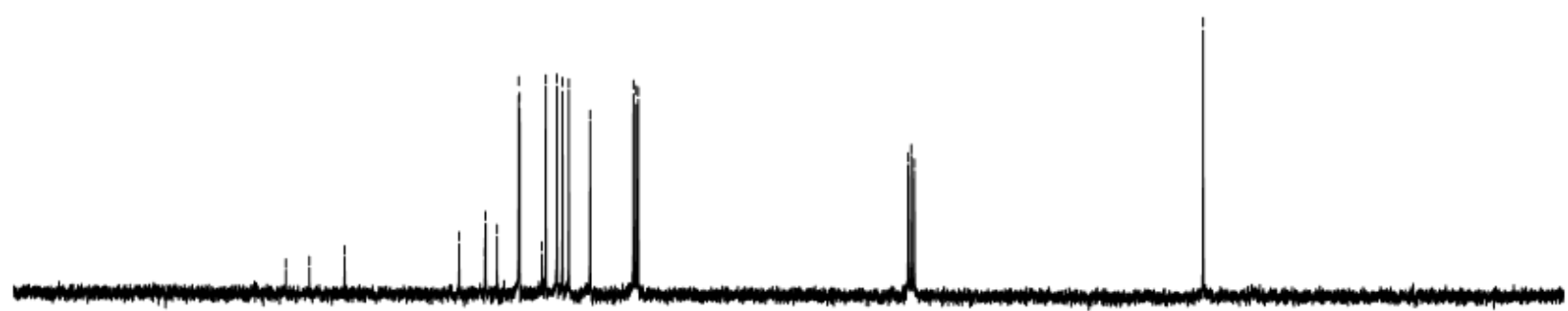

$\stackrel{7}{200}$

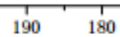

${ }_{170}^{1} \cdot \frac{1}{160}$

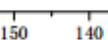

130

$100 \quad 90$

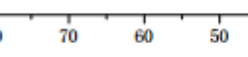

40 


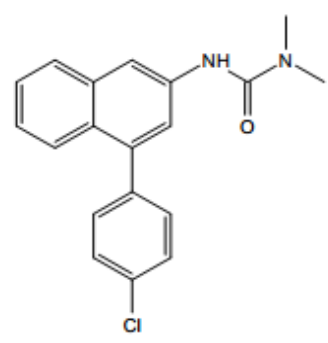

3 ag

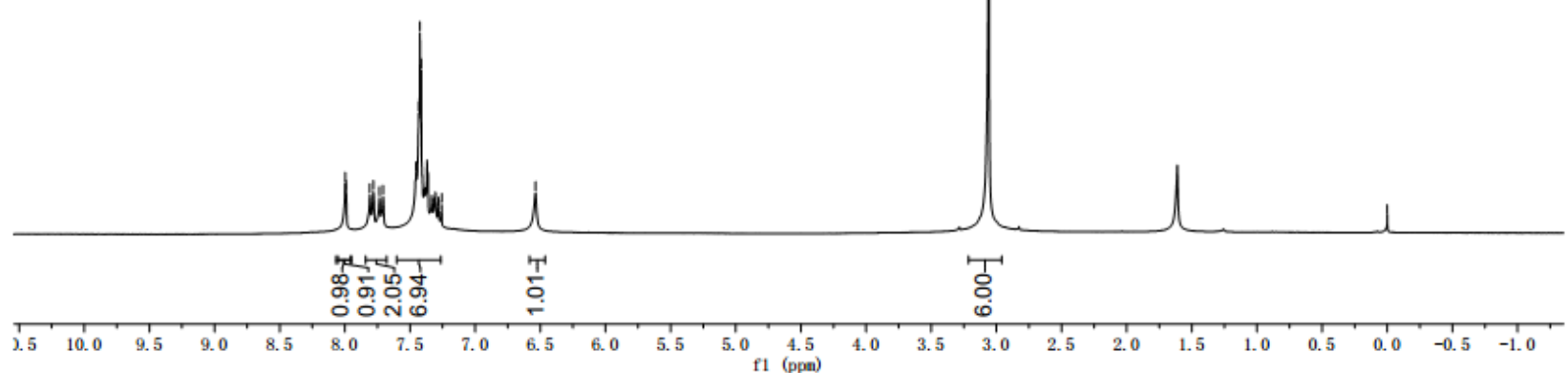

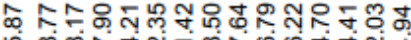

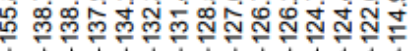

I ب

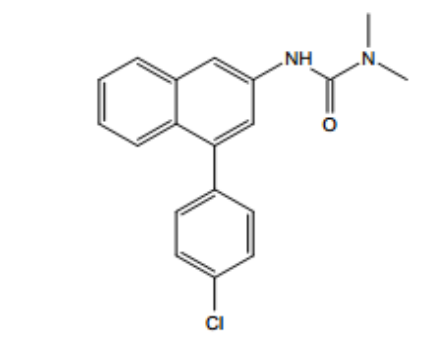

$3 a g$
ษ్

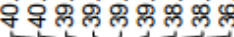

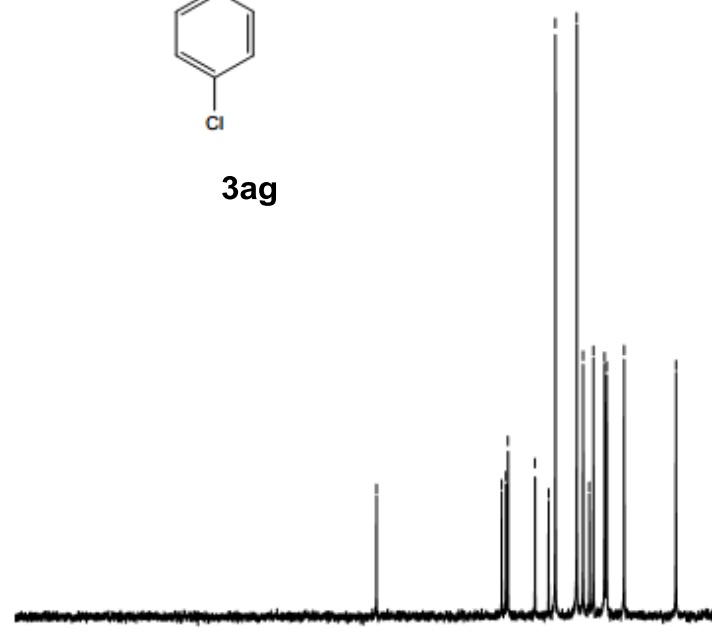

200

${ }_{190}^{1} \quad{ }^{1} 180$

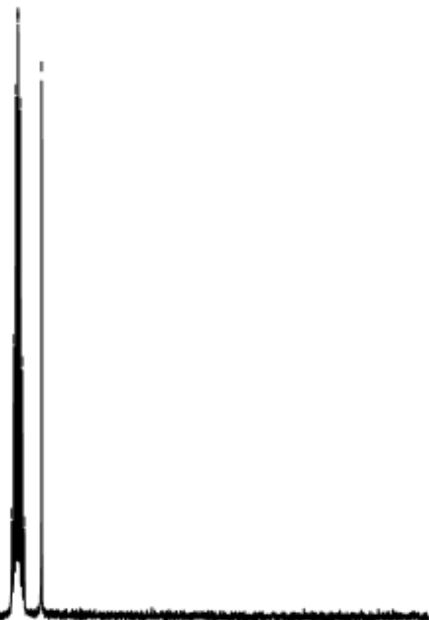




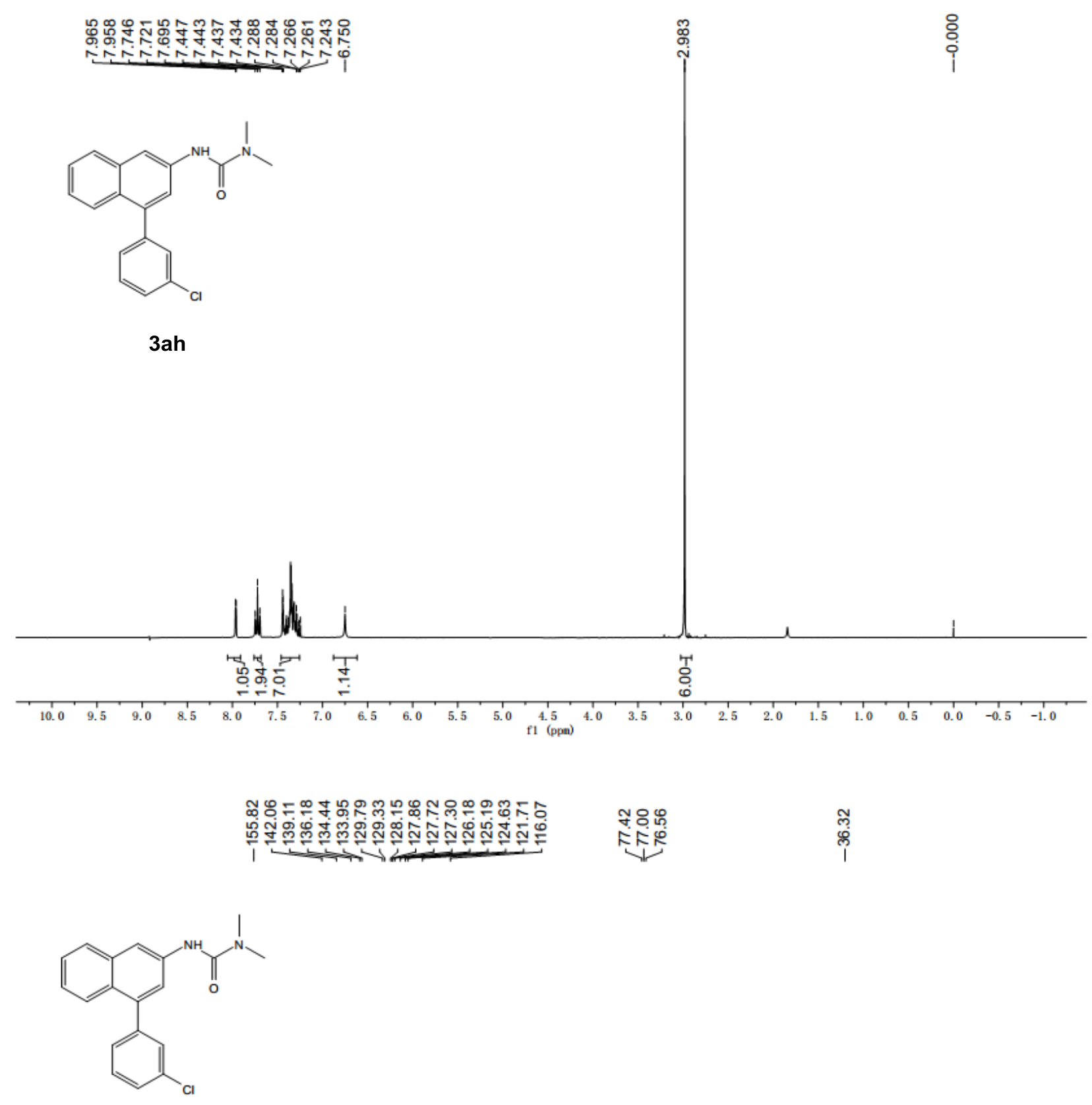

3ah
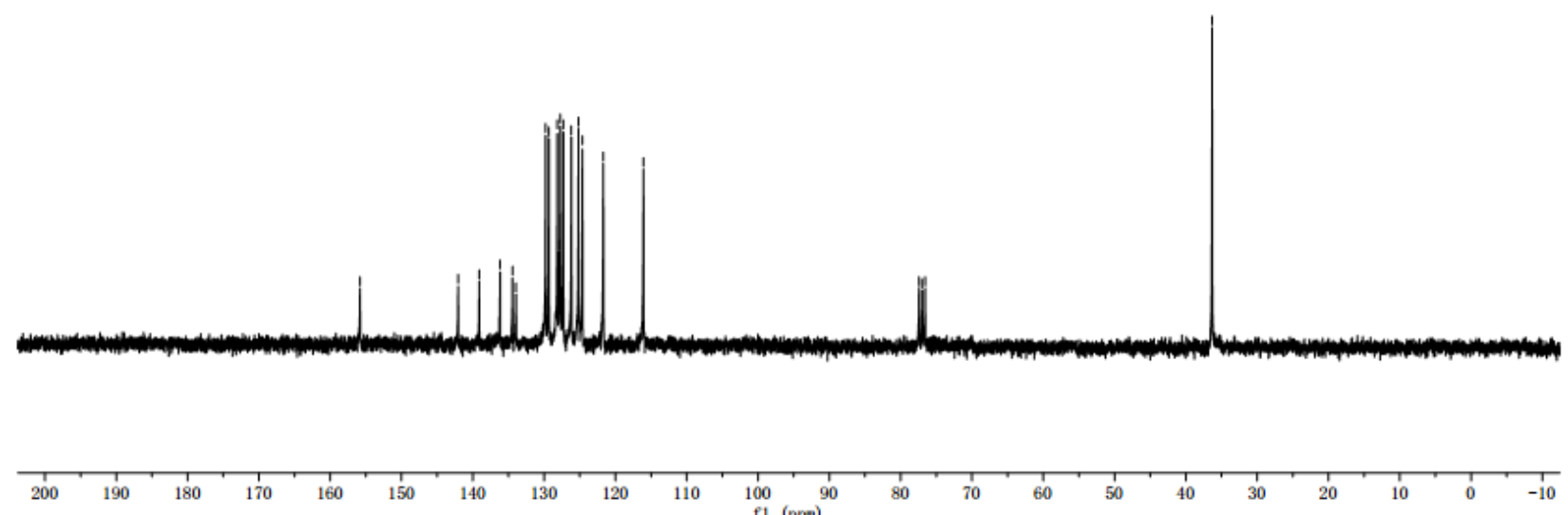


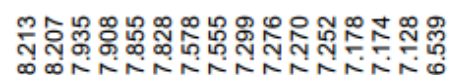

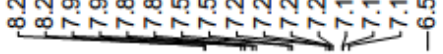

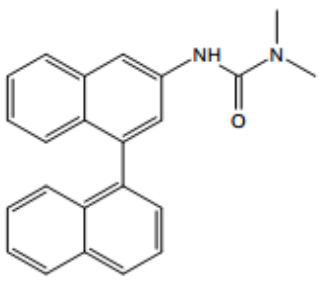

3ai

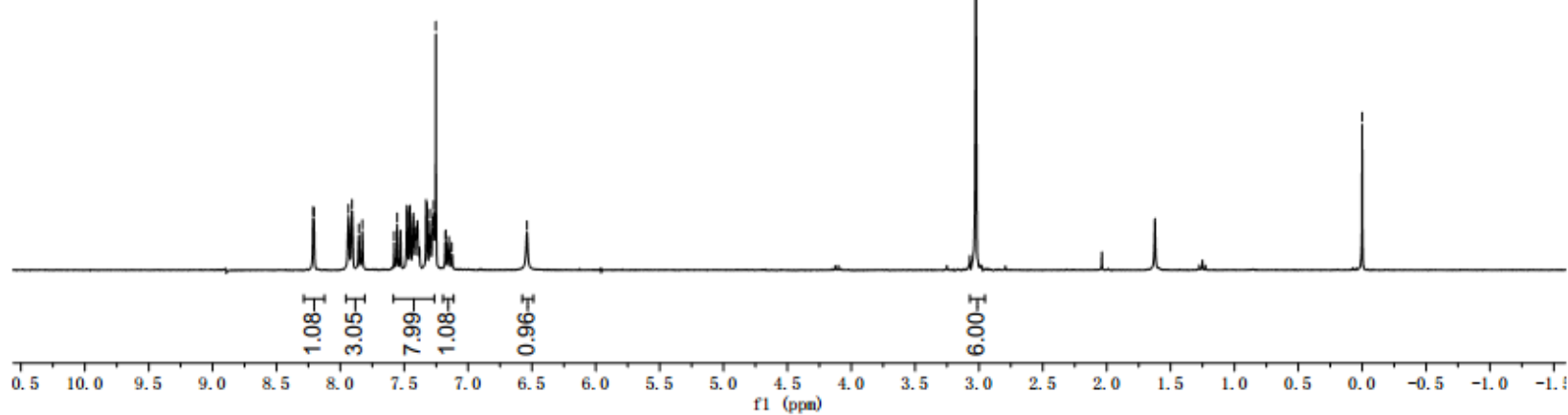

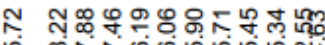

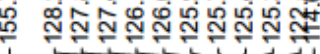

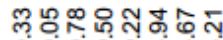

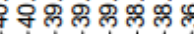

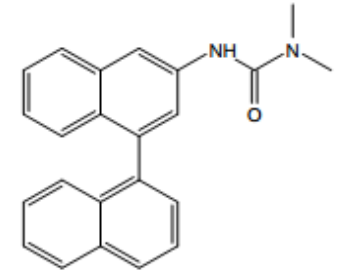

3ai

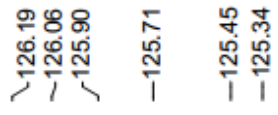

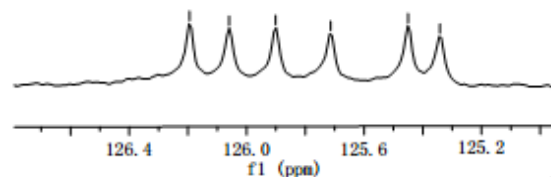

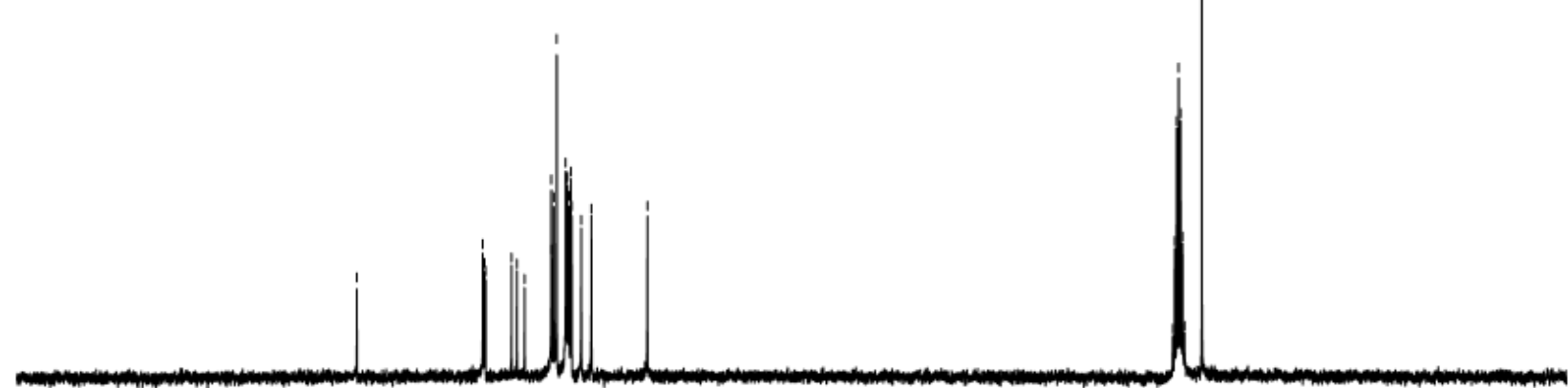

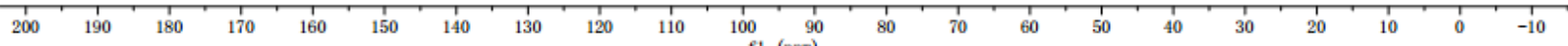




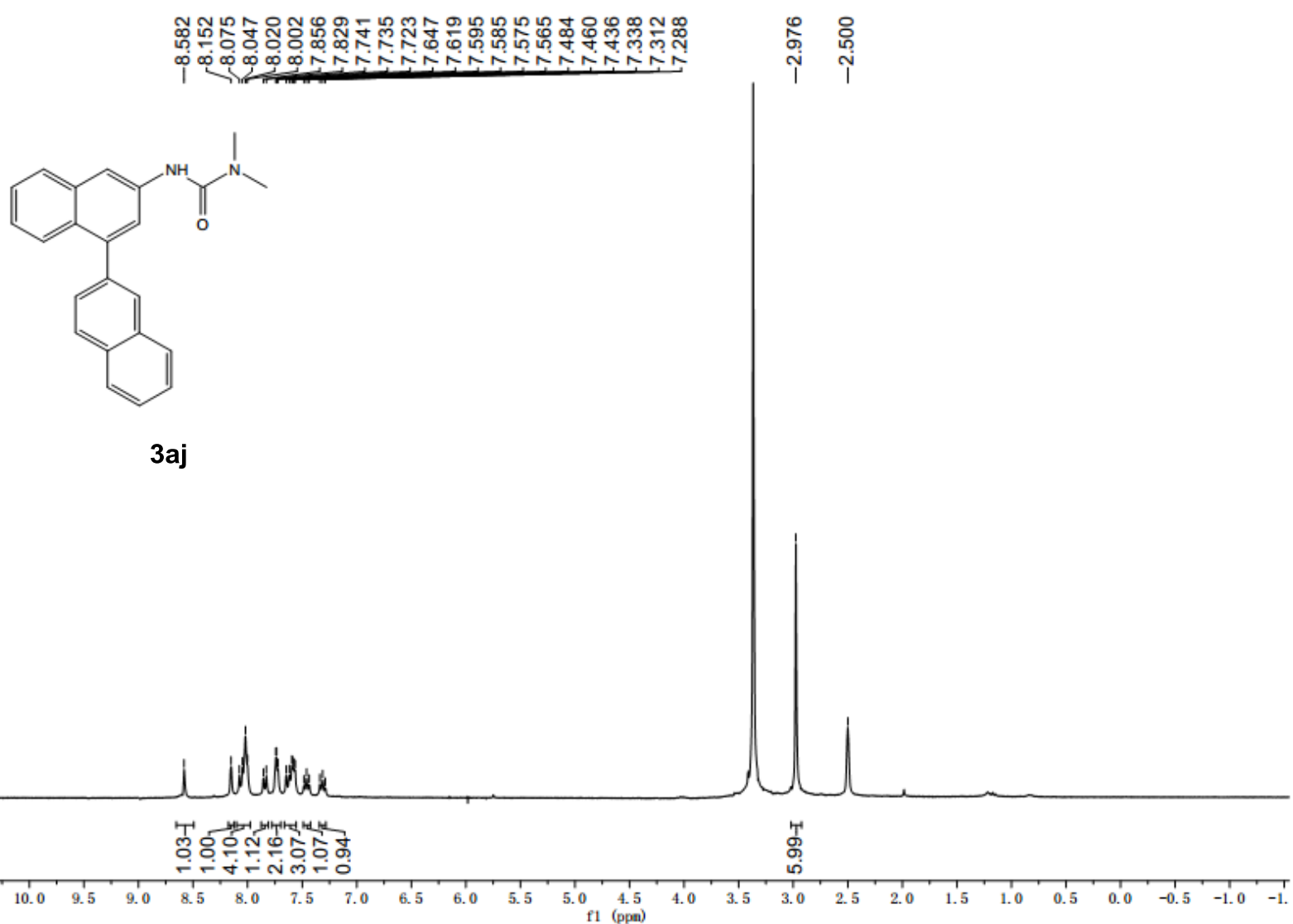

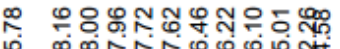

药

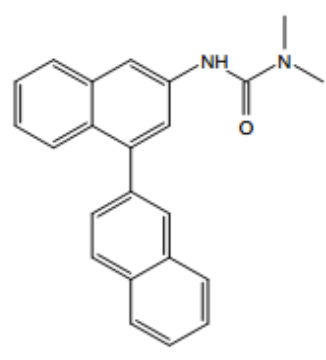

3aj

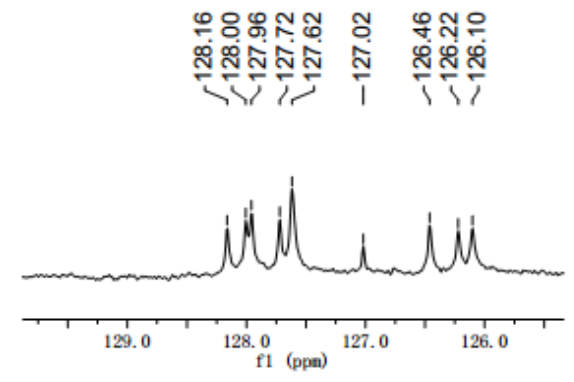

유ำ ำ 중ำ

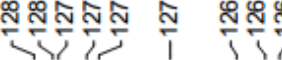

jid j

f1 (ppm)

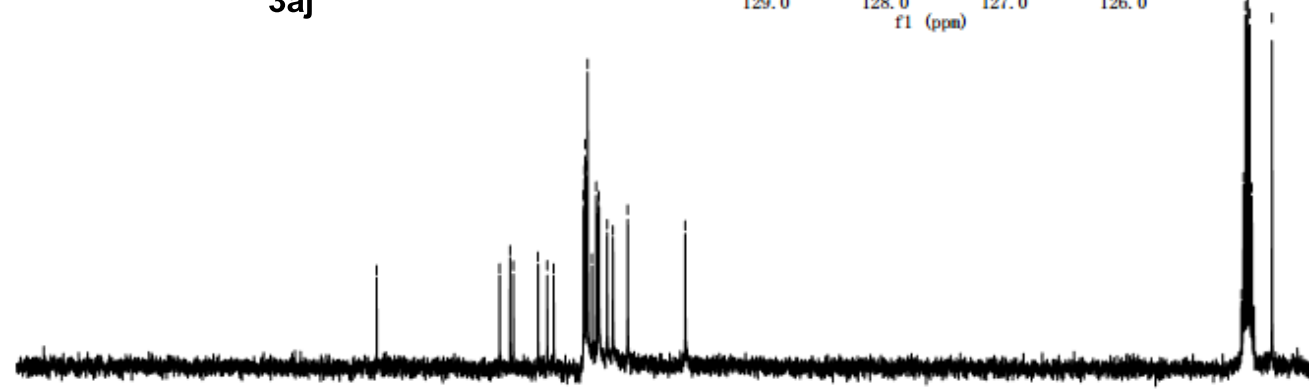

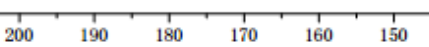

f1 (ppa) 


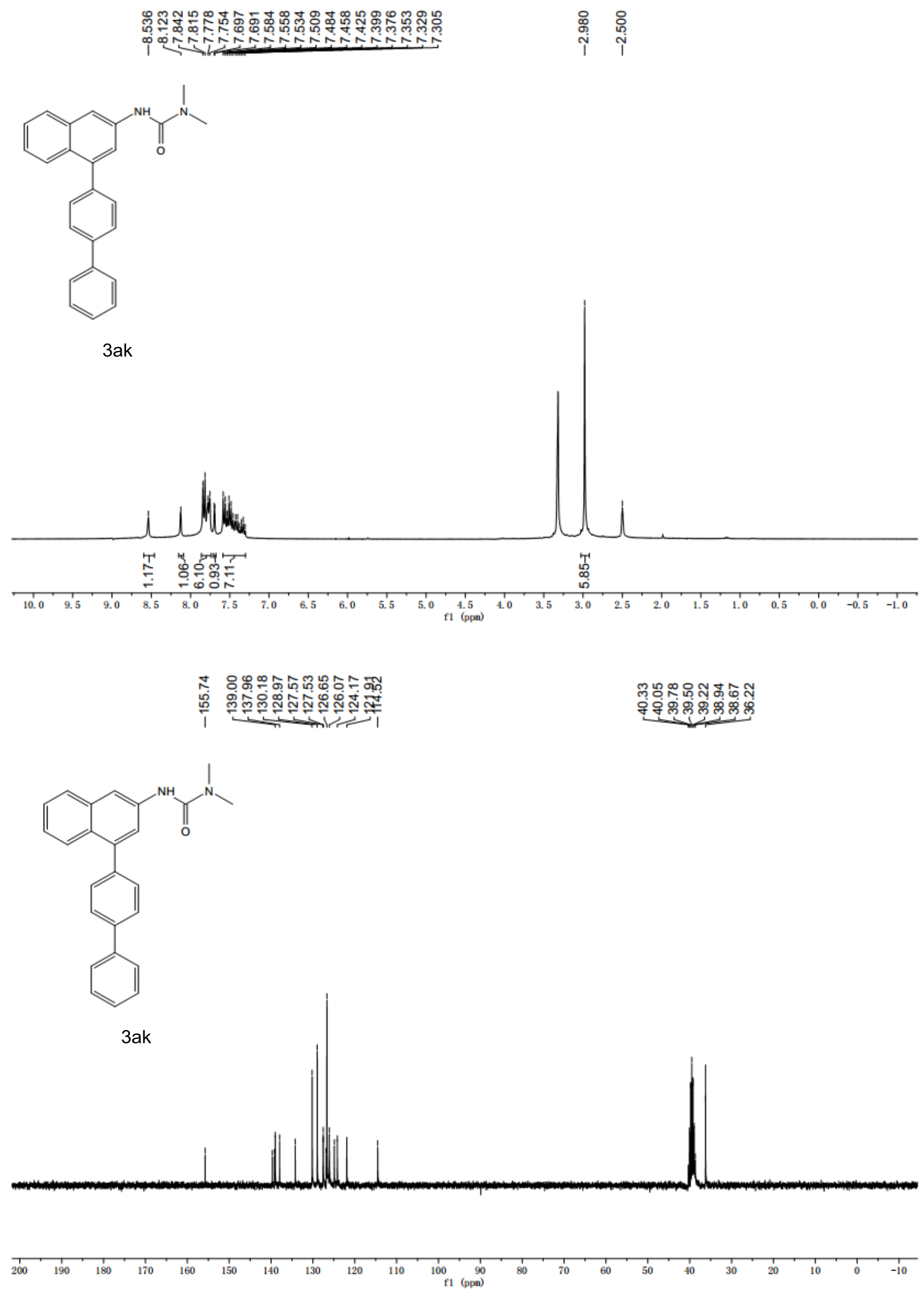



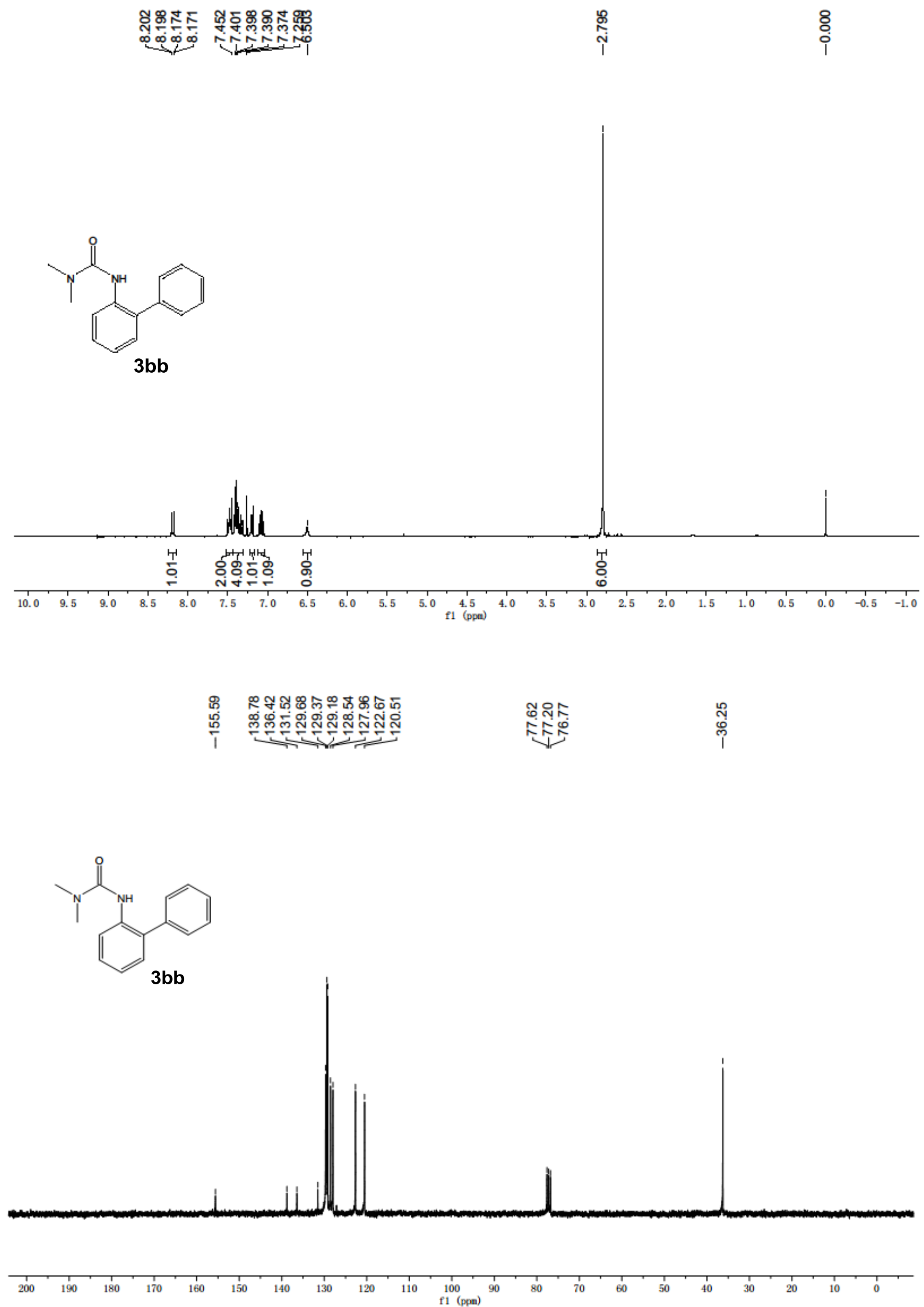


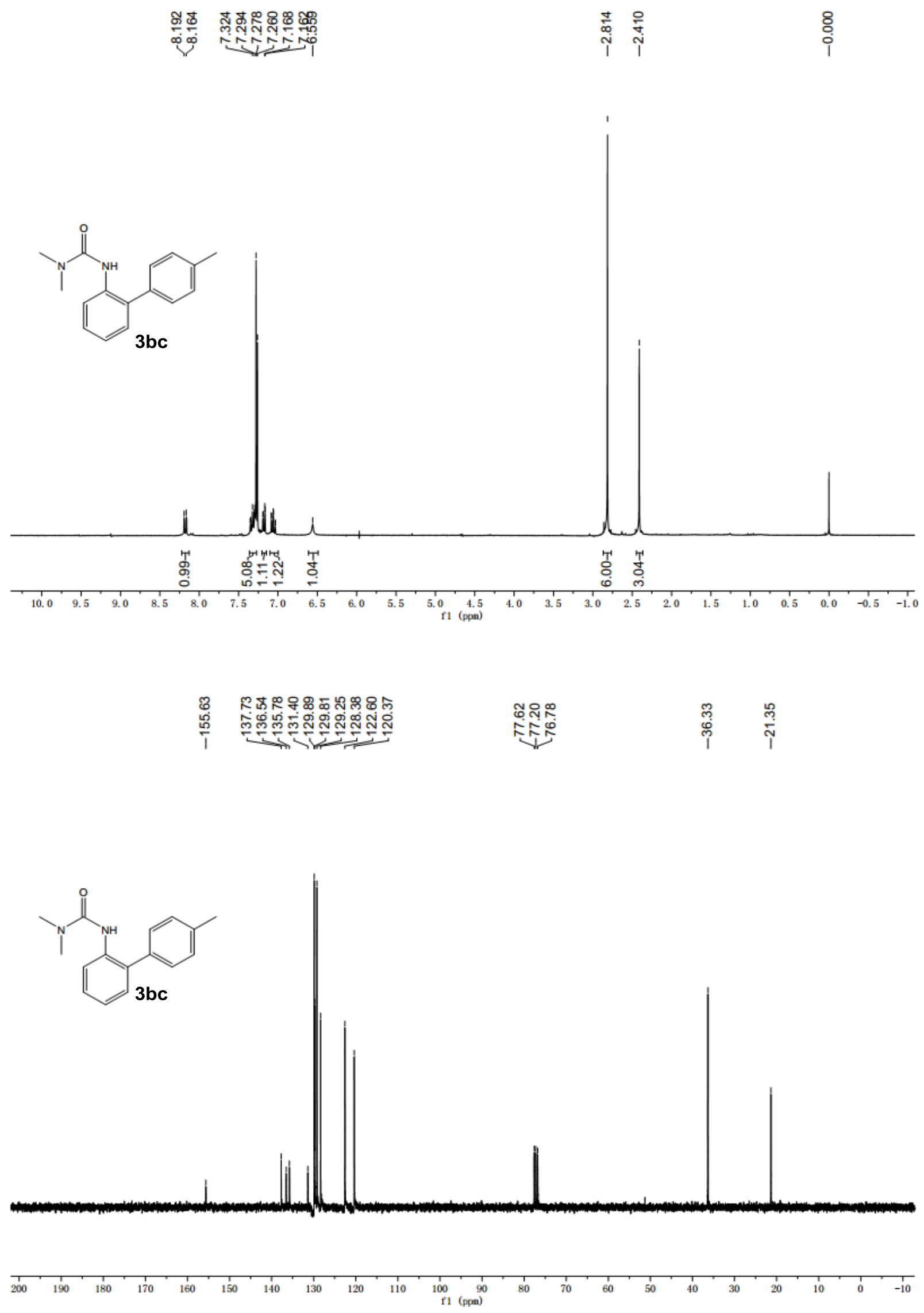




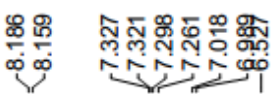

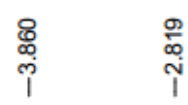

i

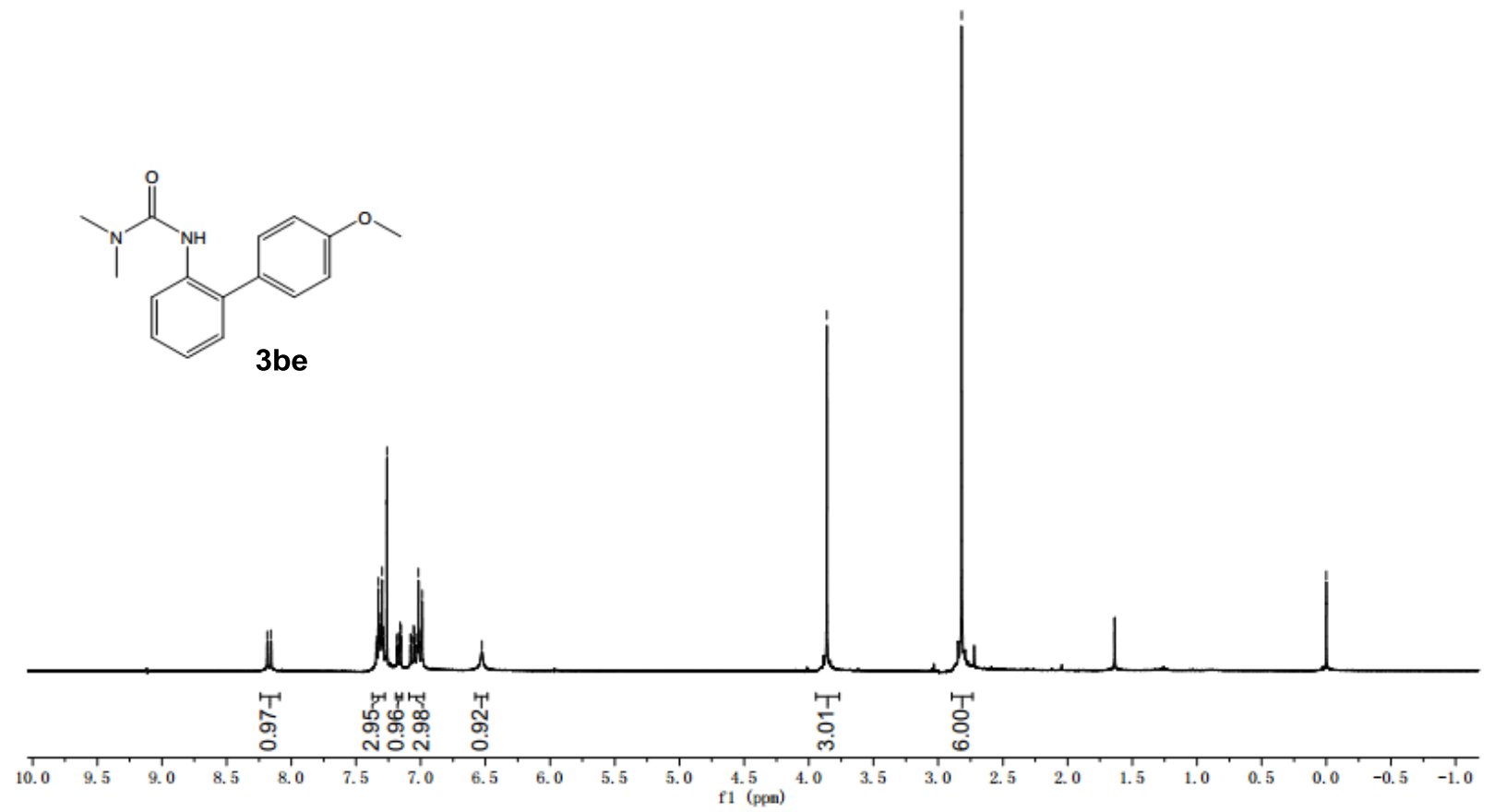

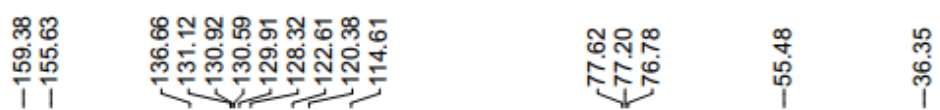

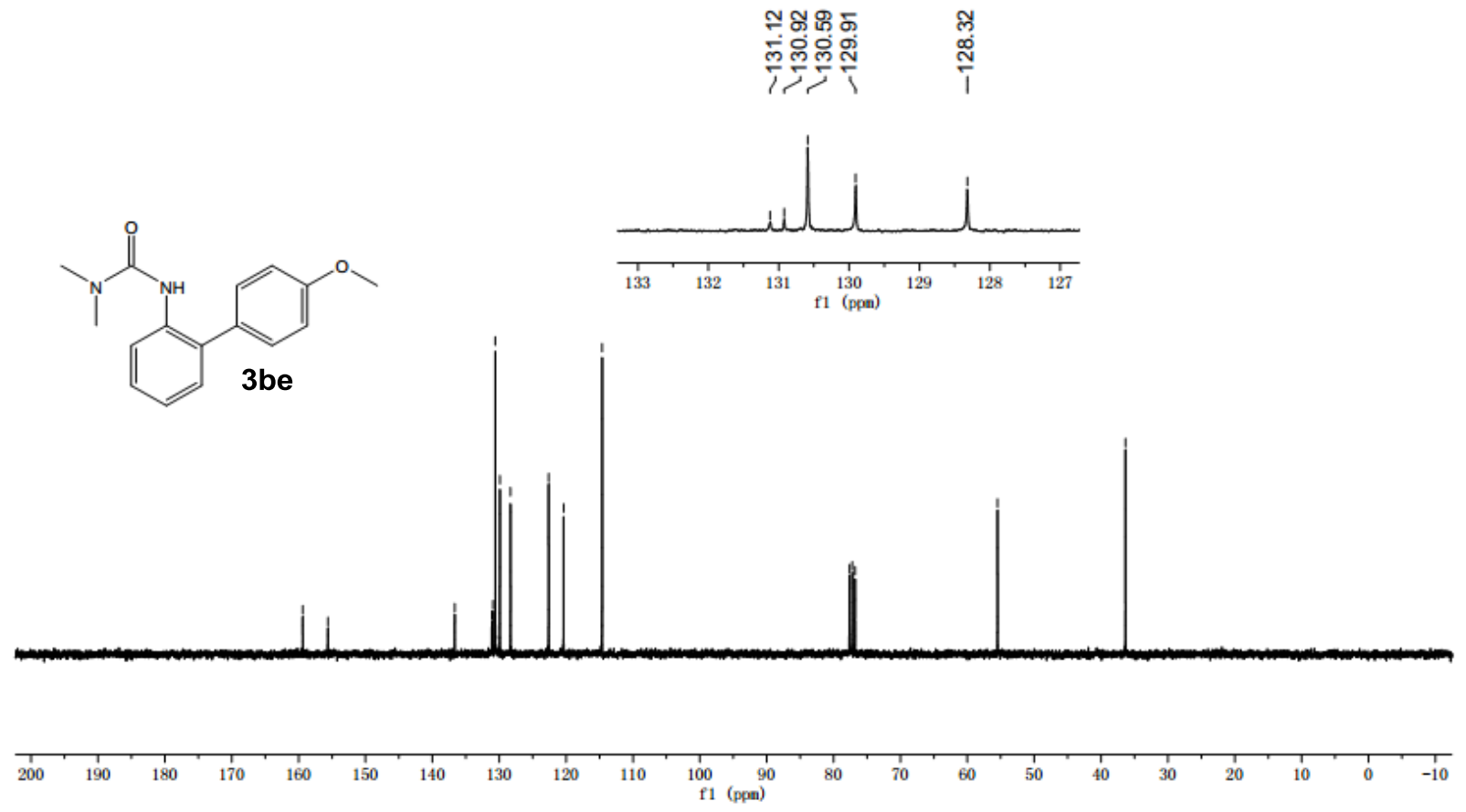




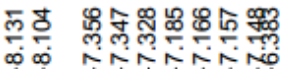
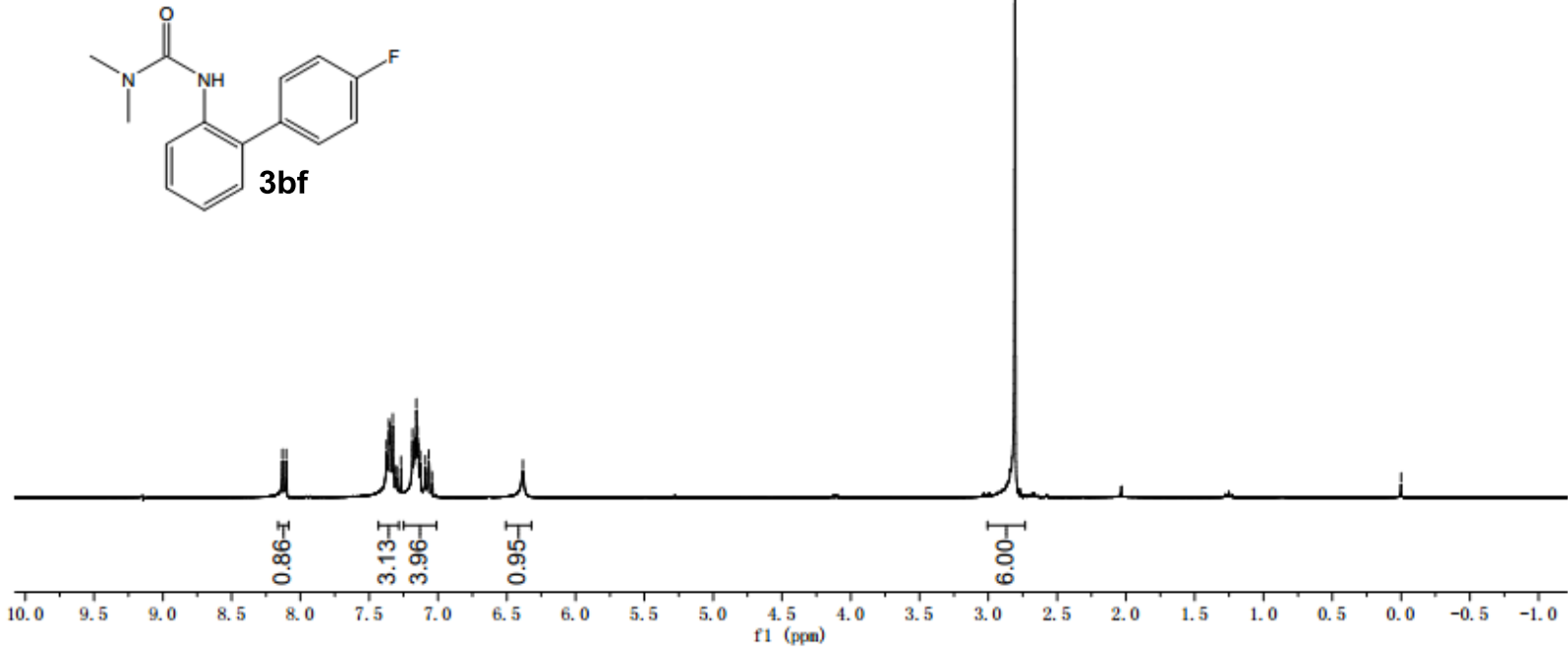

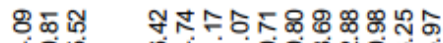

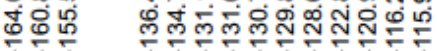

ชุำก

敊

సิ<smiles>CN(C)C(=O)Nc1ccccc1-c1ccc(Br)cc1</smiles>
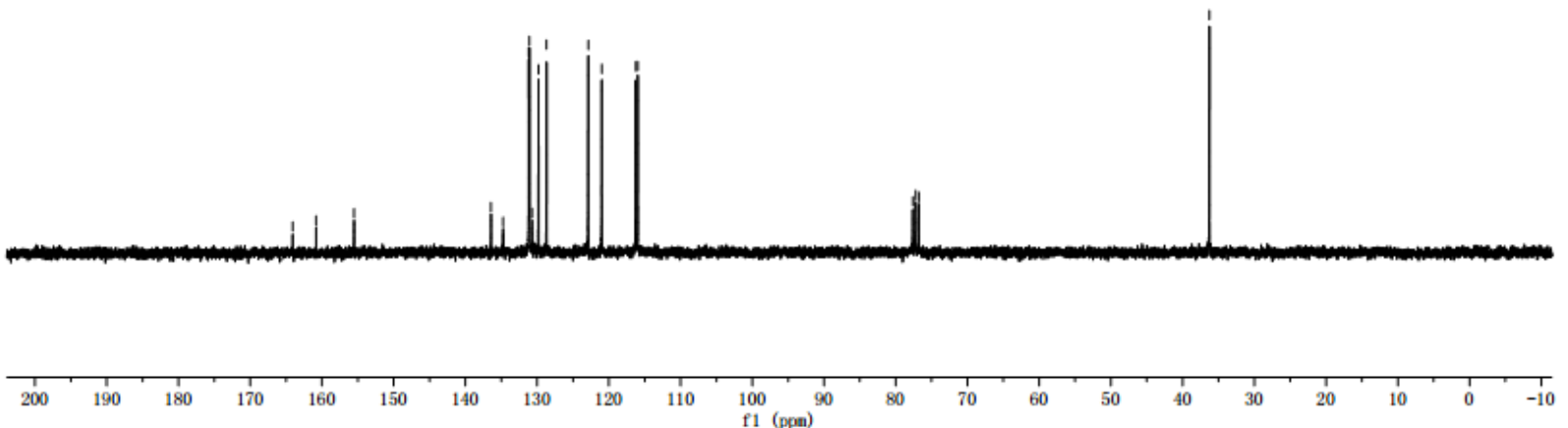


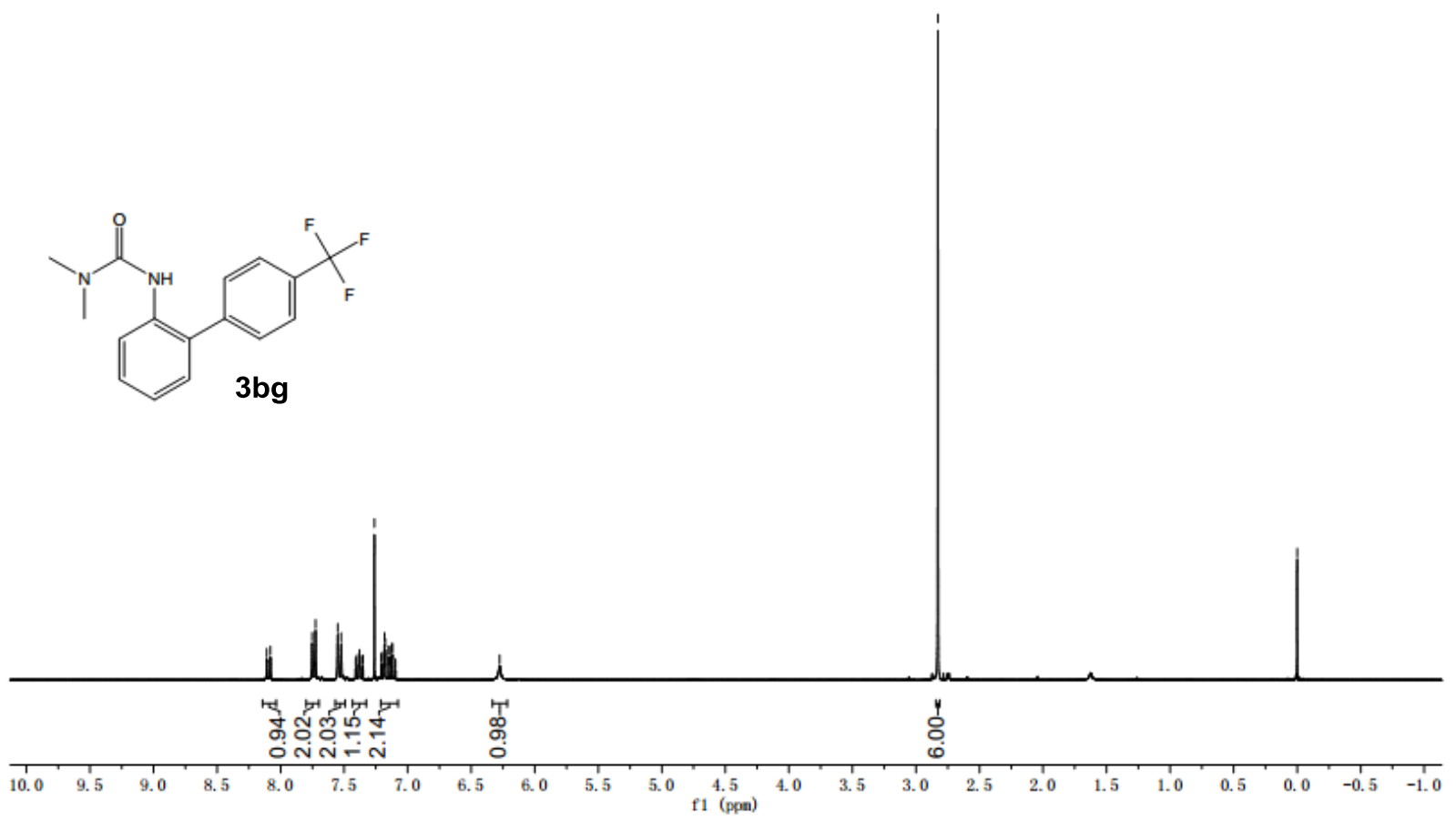

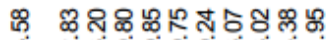

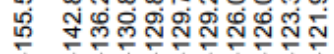

กฺฺุุ

福

岕<smiles>CN(C)C(=O)Nc1ccccc1-c1ccc(S(=O)(=O)[O-])cc1</smiles>

$3 \mathrm{bg}$

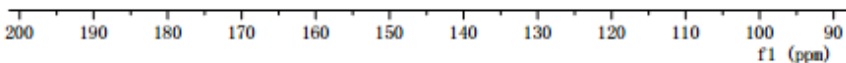




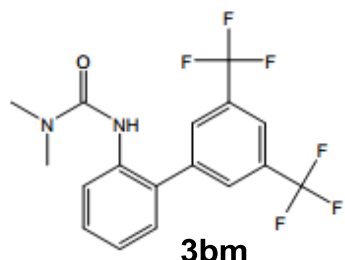

$3 b m$

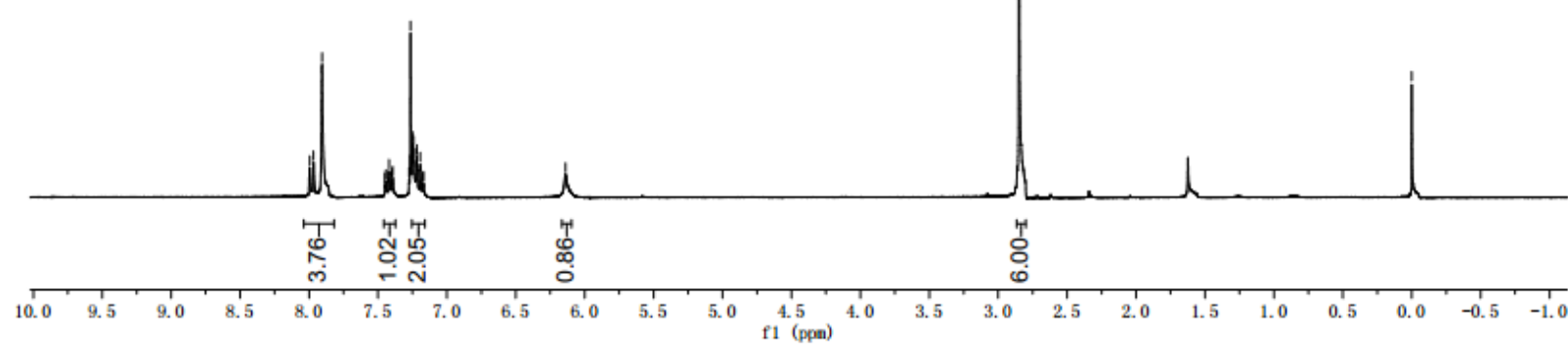

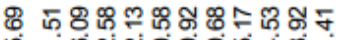

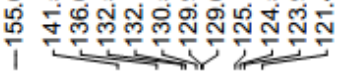

ํํำ

ल्

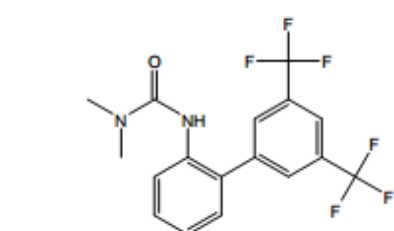

$3 \mathrm{bm}$

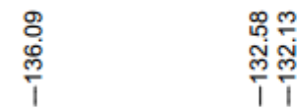

సึำ

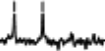
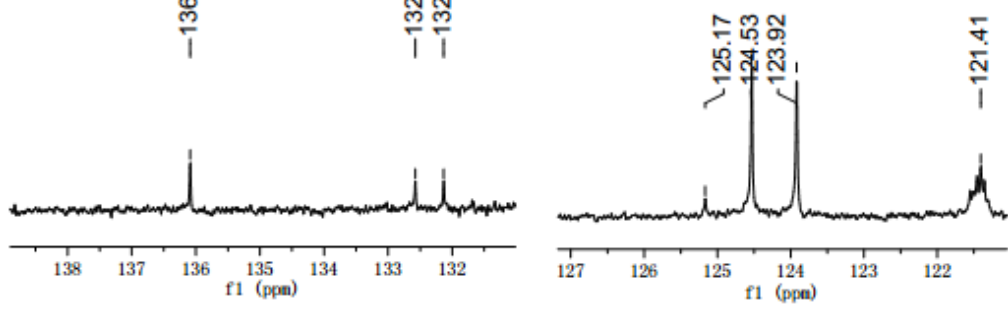

200
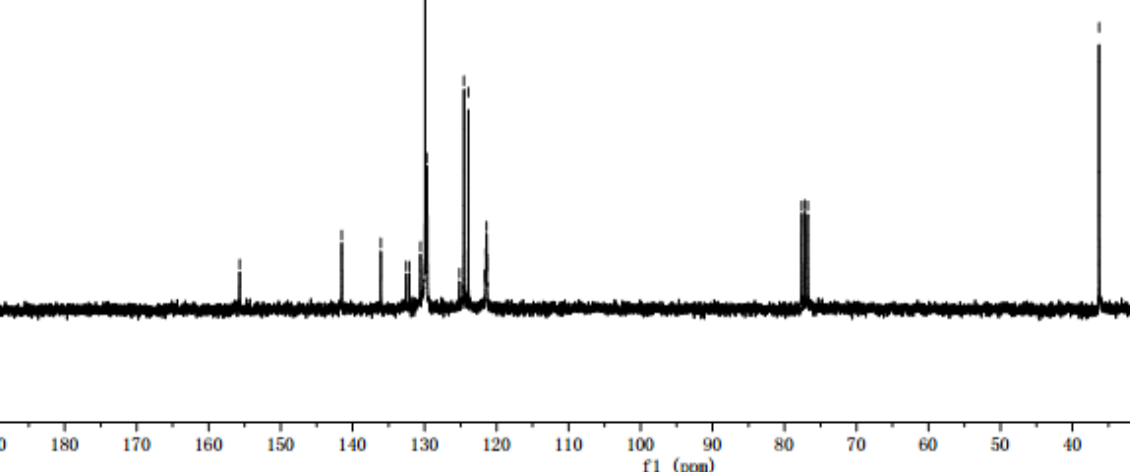


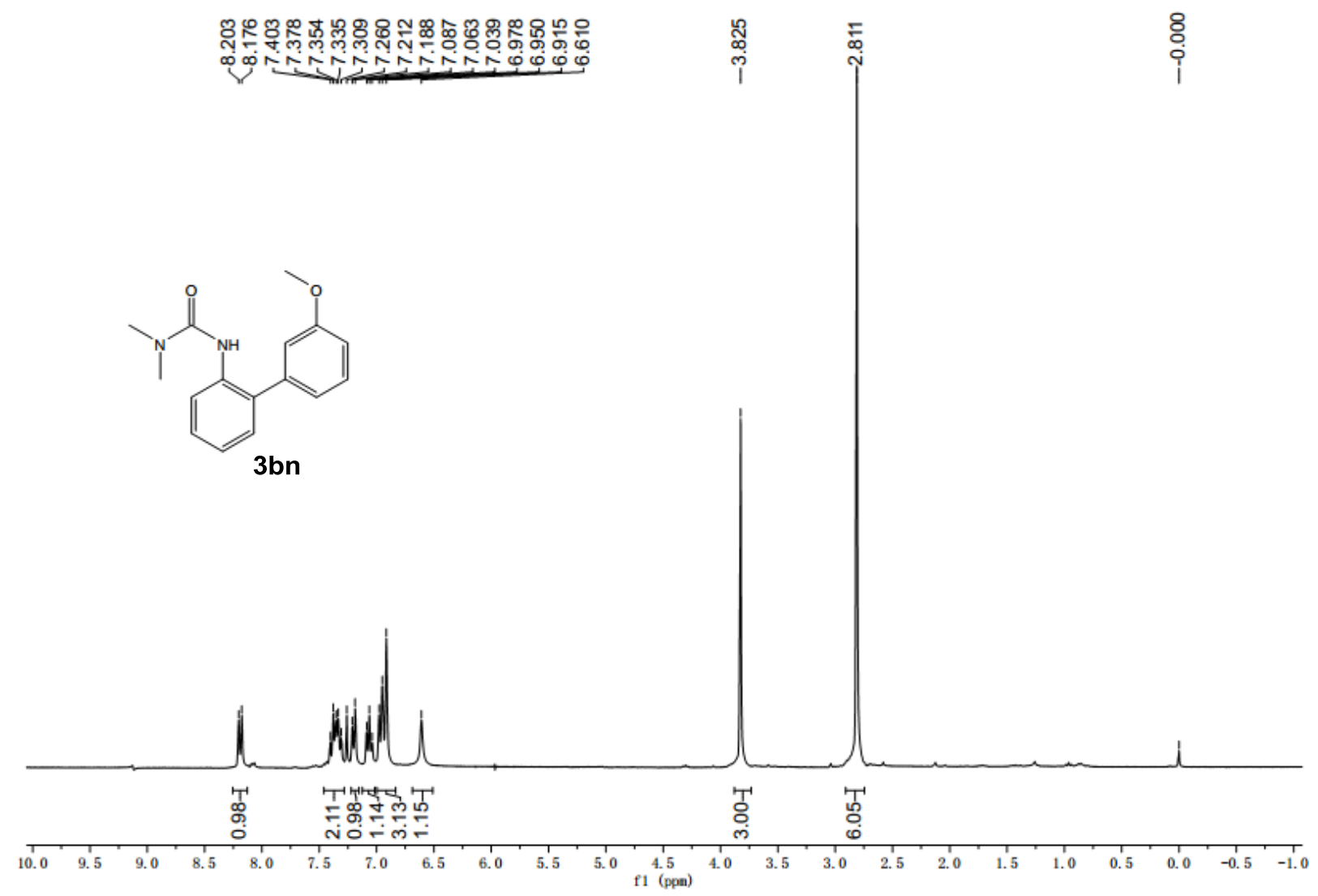

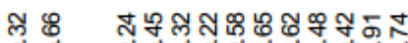

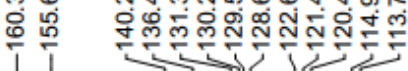

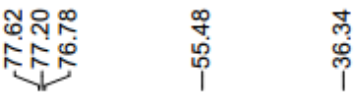
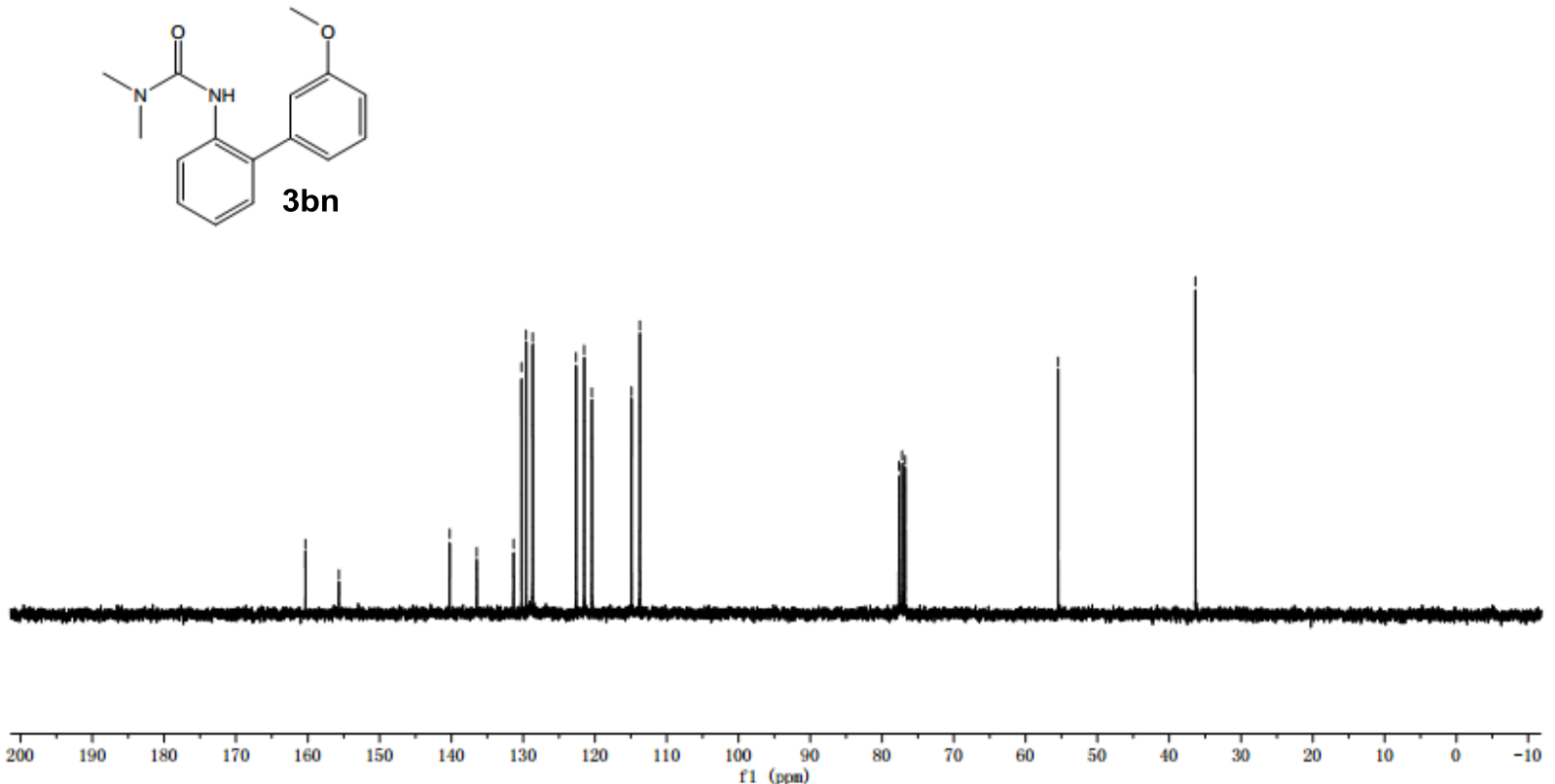


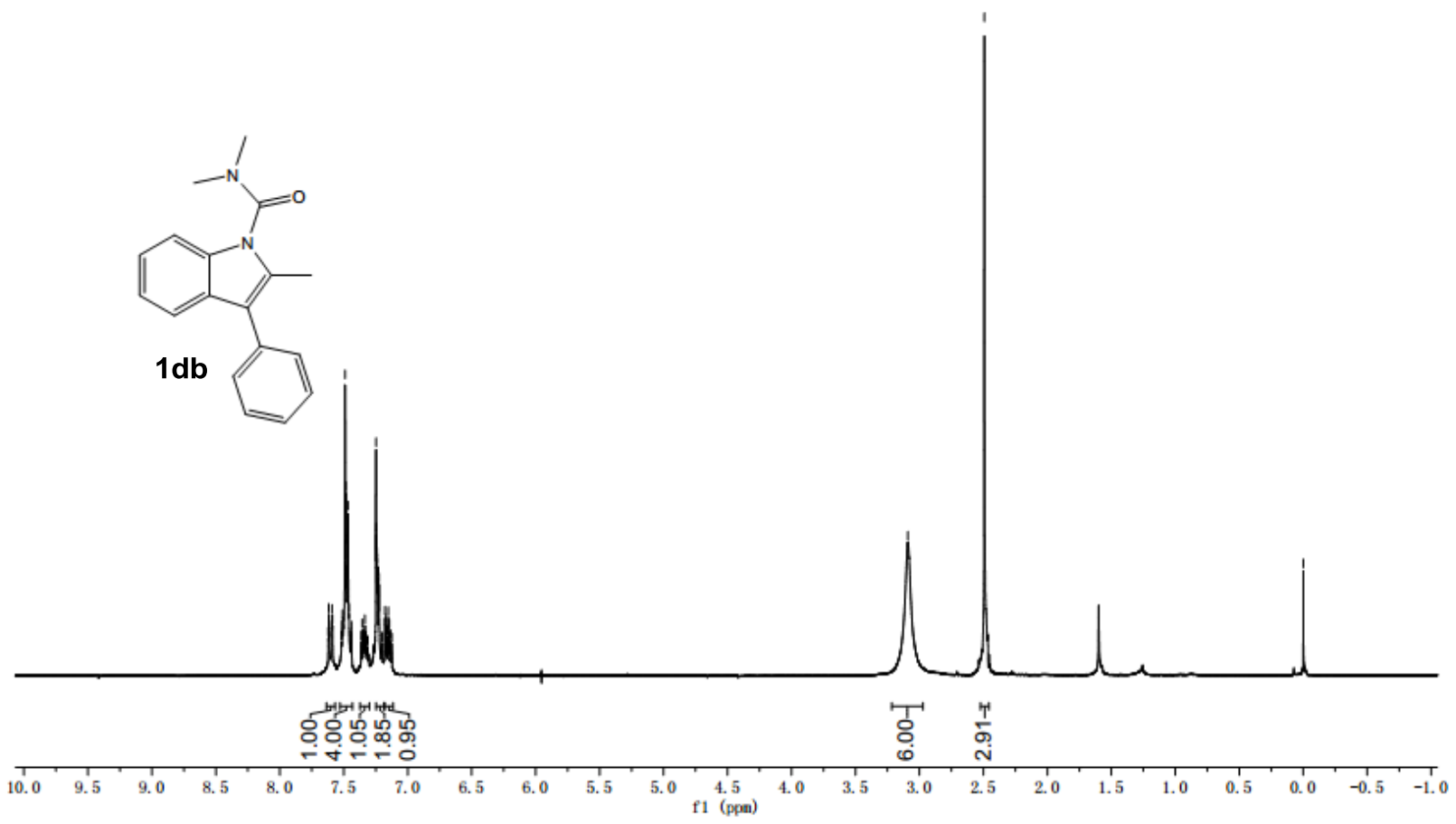

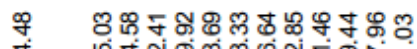

药

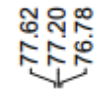

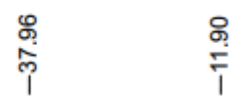

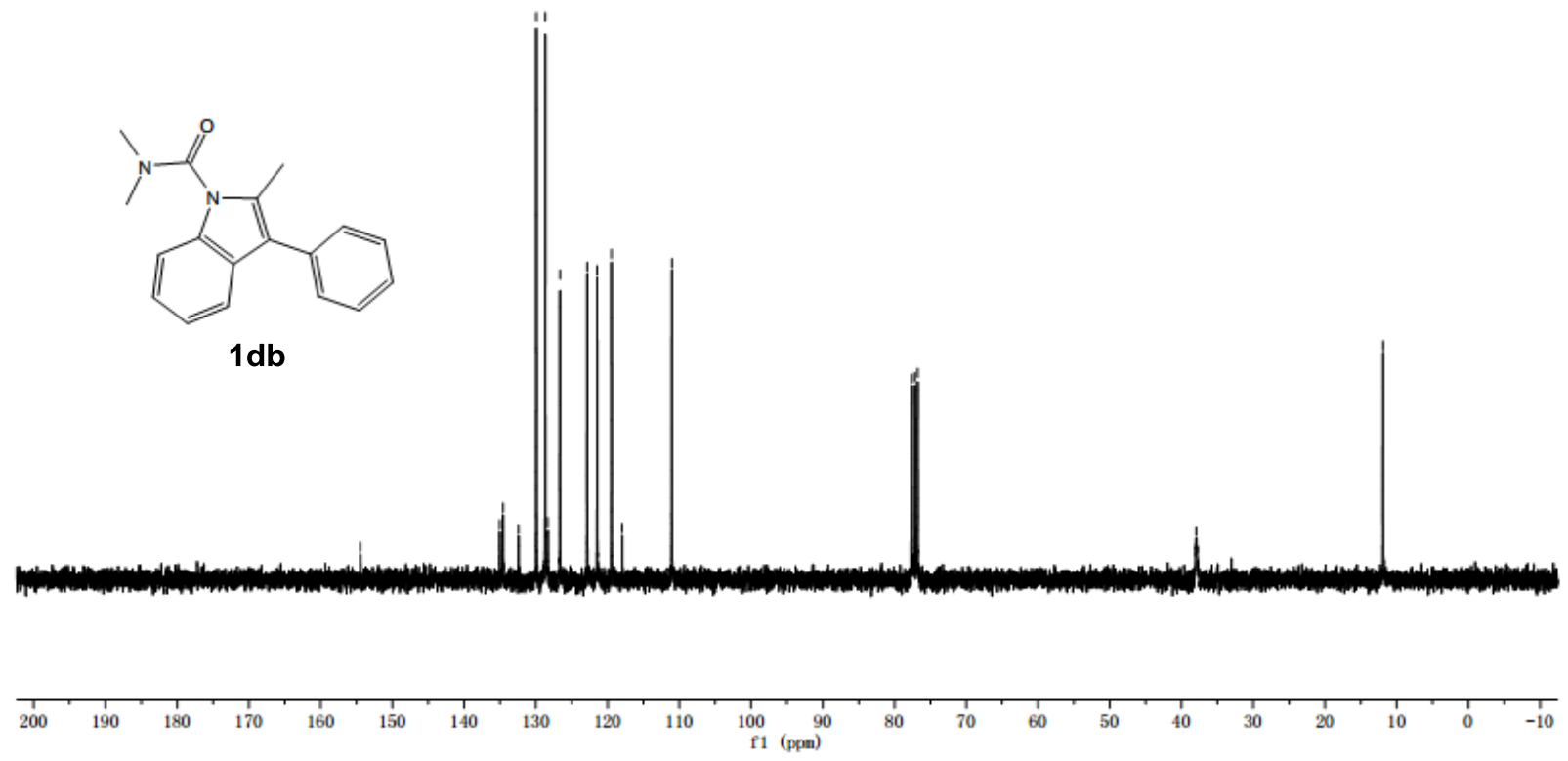




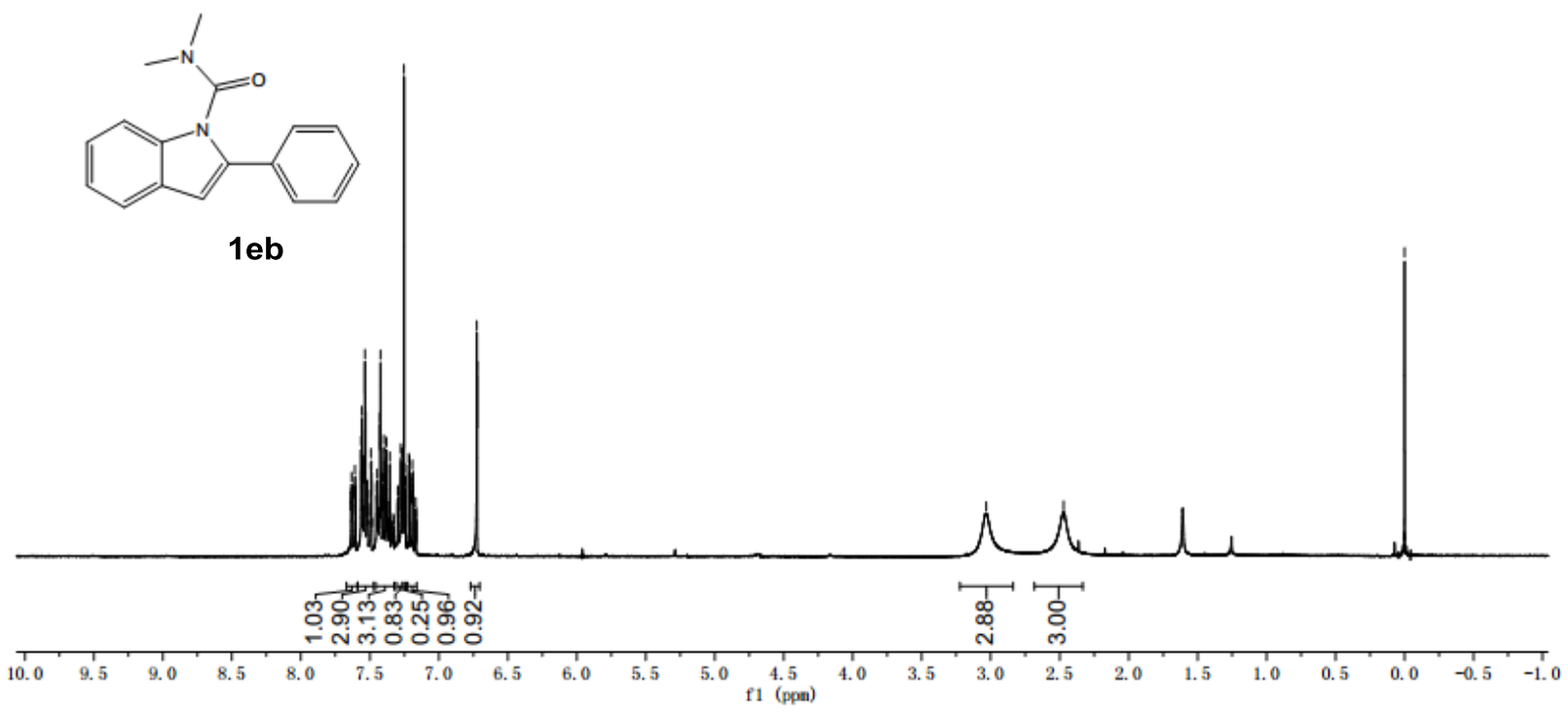

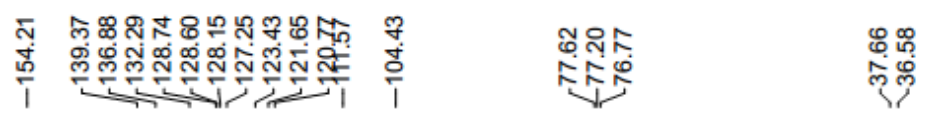

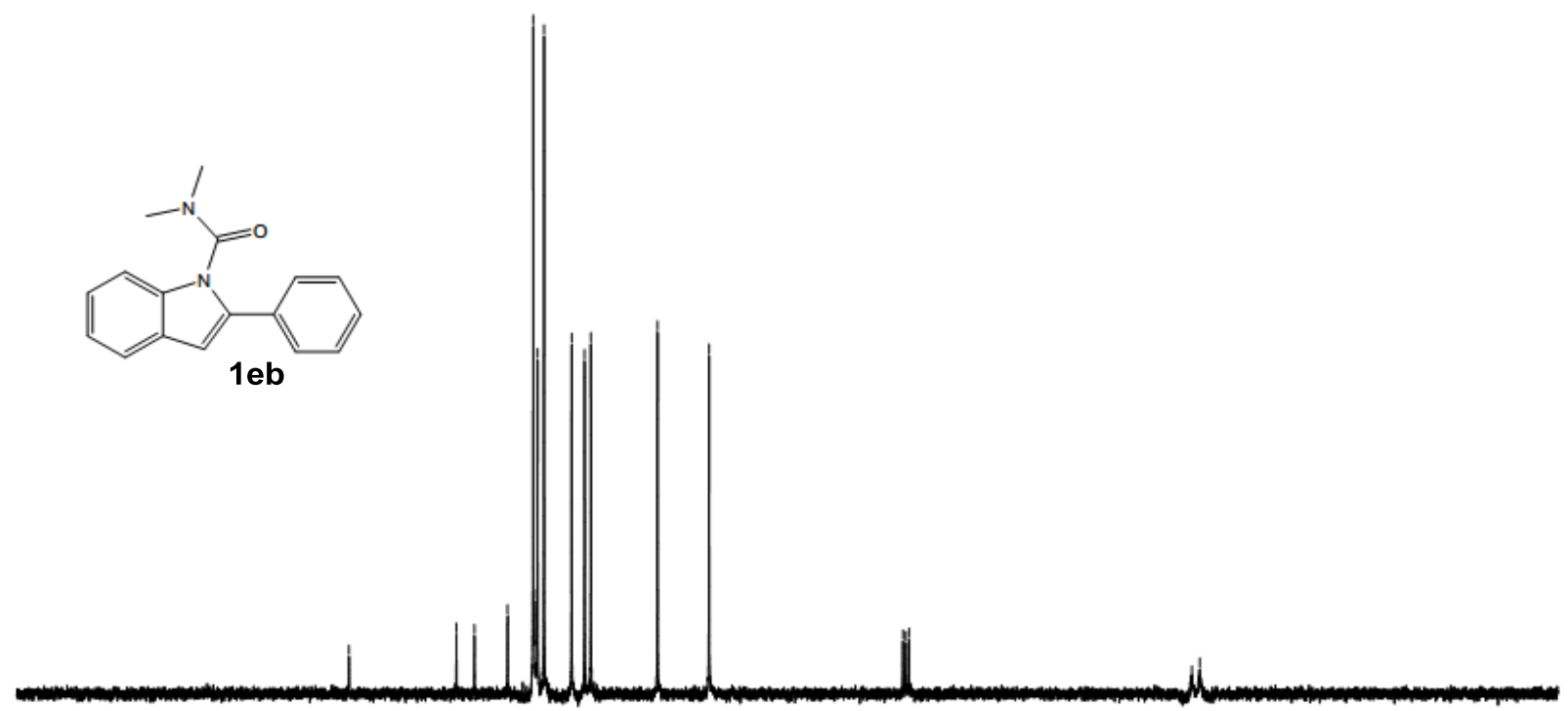

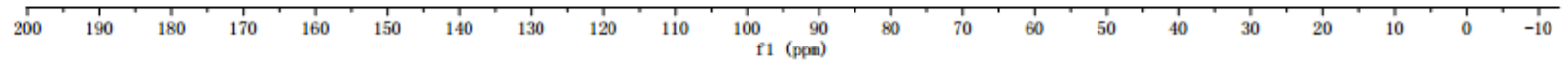



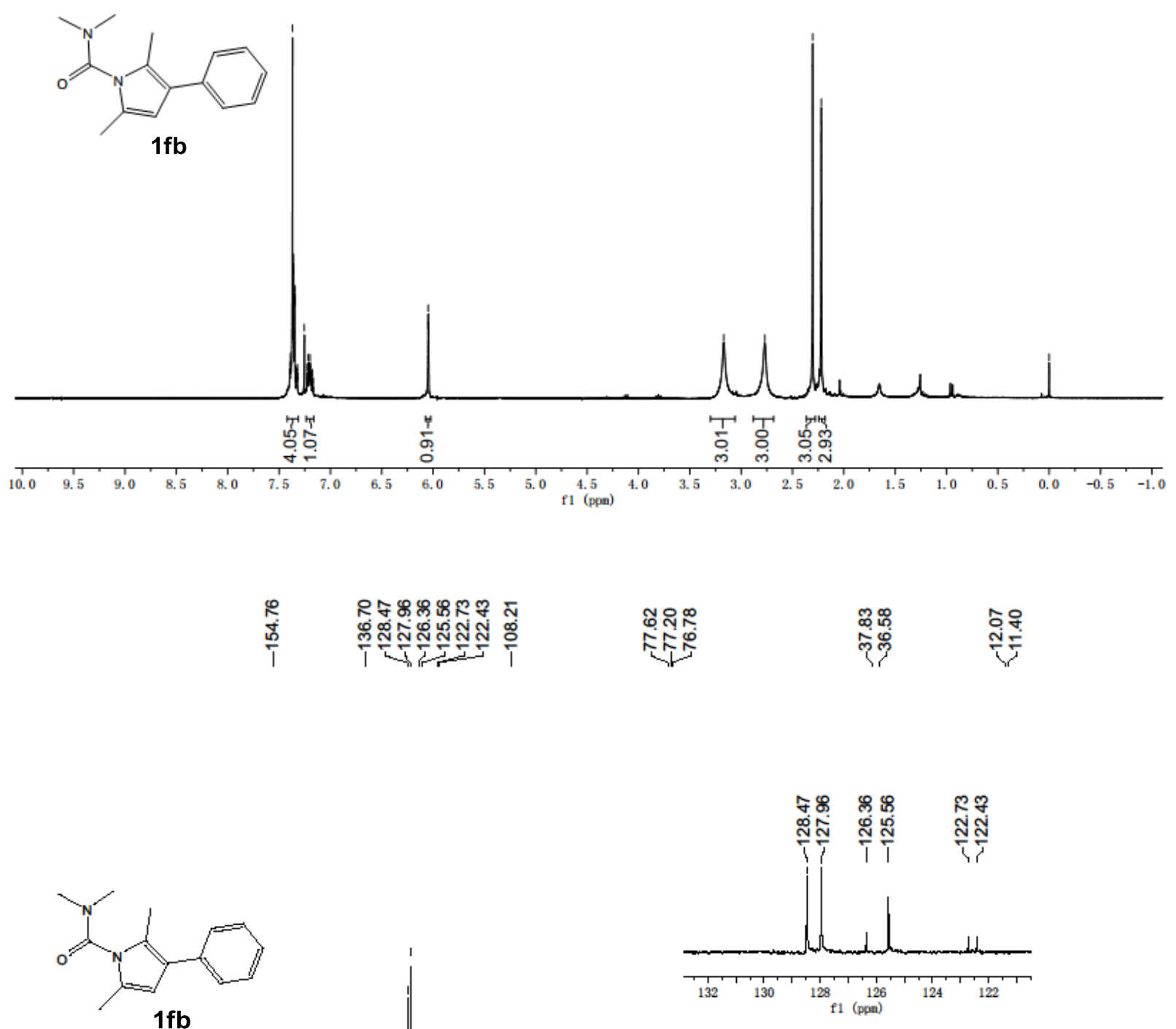

$1 \mathrm{fb}$

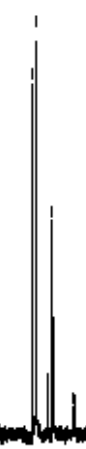

$200 \quad 190$ $\begin{array}{rrrrrrrrr}180 & 170 & 160 & 150 & 140 & 130 & 120 & 110 & 100 \\ \mathrm{f} 1 & 1 & 1 & 1\end{array}$ 


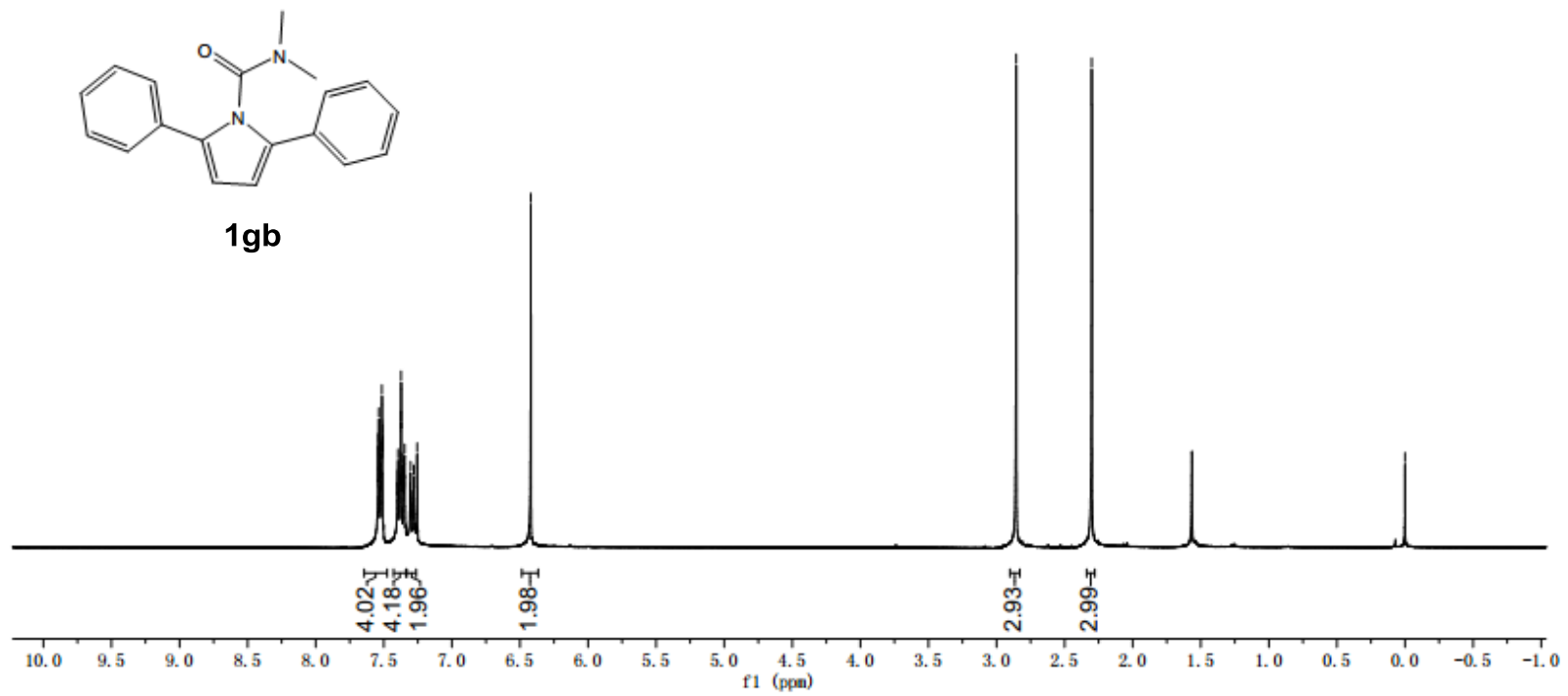

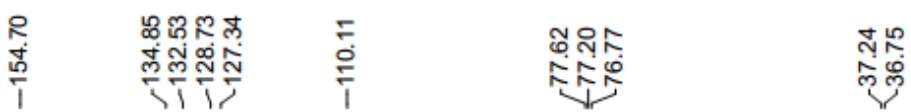
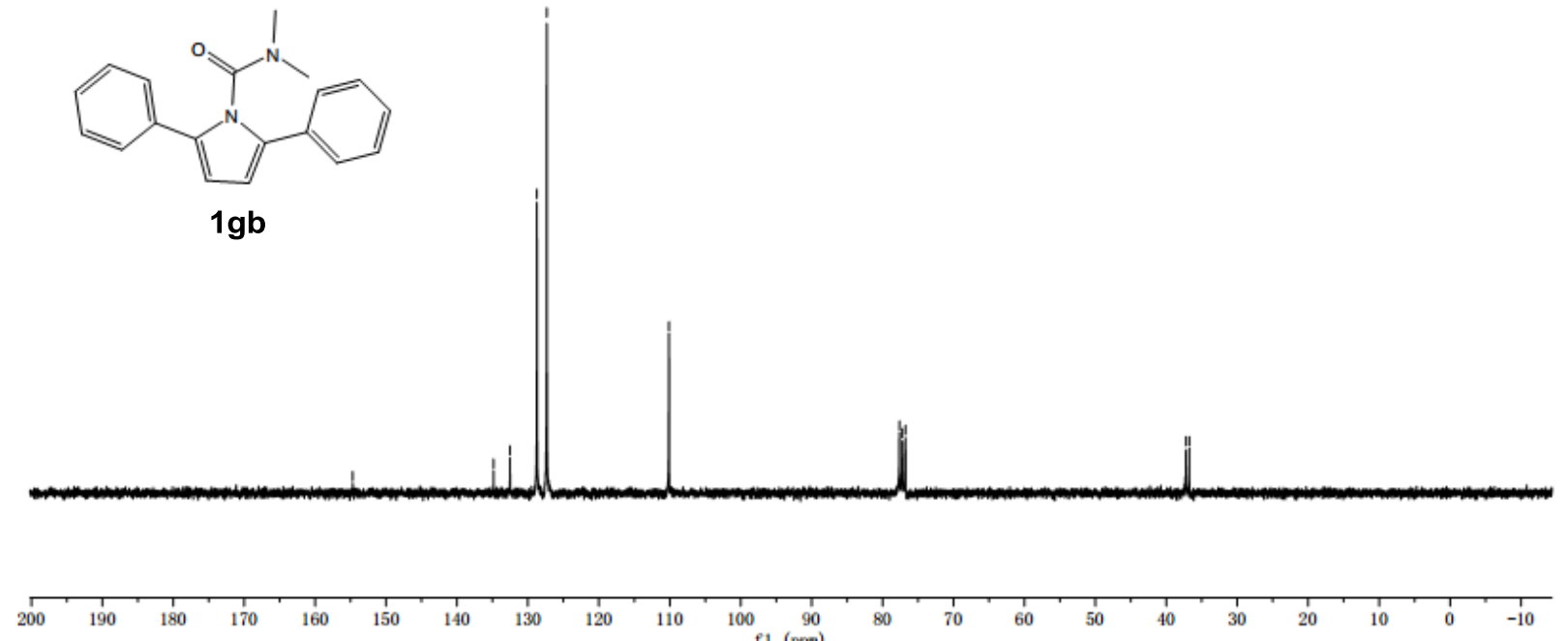